\title{
Searching for $B_{s}^{0} \rightarrow J / \psi \eta$ with the Collider Detector at Fermilab
}

\author{
William Hamish Bell \\ A thesis submitted to the Physical Sciences Graduate School \\ at the University of Glasgow \\ for the degree of \\ Doctor of Philosophy
}

Department of Physics and Astronomy

(c)William Hamish Bell

$30^{\text {th }}$ September 2002 


\begin{abstract}
This thesis describes a detailed search for the decay $B_{s}^{0} \rightarrow J / \psi \eta$, within data taken from $p \bar{p}$ collisions at $\sqrt{s}=1.8 \mathrm{TeV}$.

An introduction of particle physics and the motivations of the subject area are given. The Standard Model, the current theoretical model to describe three of the four observable forces and their interactions with the fundamental particles, is briefly summarised. Aspects of the weak interaction between quarks are then discussed in more detail. The mixing matrix and the accompanying model of quark mixing is introduced. Attention is then focused on $\mathrm{CP}$ violation and its expected limits within the Standard Model. Having summarised the theory in general, the specific motivation for this thesis is given.

The data studied were recorded with the Collider Detector at Fermilab (CDF): a complex detector composed of many different component parts, positioned at one of two interaction points on the Tevatron accelerator. The accelerator used to produce the colliding beams is discussed in its 1992-1996 configuration. Description is given of each stage of the acceleration process, from production of $\mathrm{H}^{-}$ions, to the collision architecture within the CDF experimental hall. From the proton and anti-proton beams data were collected from many different pieces of the CDF detector. The CDF detector is introduced and a summary of the component parts is given. Specific parts of the detector from which output data were used are described, these include: tracking, central calorimetry and muon chambers.
\end{abstract}

Proton anti-proton collisions contain many different physics processes. From these processes many are of lesser interest. The trigger logic specific to selecting a sample of data rich in processes relevant to this particular study is described in detail.

The analysis method proceeds as follows. simulation program used to model acceptances of $B^{+} \rightarrow J / \psi K^{+}$and $B_{s}^{0} \rightarrow J / \psi \eta$ samples is described. Within this description theoretical inputs and assumptions made are given in detail. Constraints made on each generated sample are then outlined. From the simulation, the discussion turns to the reconstruction of $B^{+} \rightarrow J / \psi K^{+}$and the search for $B_{s}^{0} \rightarrow J / \psi \eta$ within the CDF data. All stages of data selection are discussed. The analysis then turns to acceptance and efficiency factors considered. Specific effort is made to fully describe the 
photon reconstruction efficiency. Photon reconstruction efficiency is studied in the CDF environment by introducing a data based Monte Carlo program. This detailed simulation is discussed and final photon reconstruction efficiencies are given.

Systematic uncertainties are analysed in detail. The generator level simulation is used to provide error propagation for acceptance and efficiency parameterisations introduced. Systematic uncertainties from the analysis of data are also given.

The results of the $B^{+} \rightarrow J / \psi K^{+}$reconstruction and the search for $B_{s}^{0} \rightarrow J / \psi \eta$ are given. From an integrated luminosity of $110 \mathrm{pb}^{-1}, 1178171 \mathrm{~J} / \psi \rightarrow \mu^{+} \mu^{-}$events were collected. $490 \pm 23 \mathrm{~B}^{+} \rightarrow J / \psi K^{+}$events were isolated. From the sample of $J / \psi \rightarrow$ $\mu^{+} \mu^{-}$events a branching ratio limit of $\mathcal{B}\left(B_{s}^{0} \rightarrow J / \psi \eta\right)<6.3 \times 10^{-3}$ is set at a $90 \%$ confidence limit. Finally conclusions are made and future prospects of this decay channel are discussed. 


\section{Preface}

This thesis describes an analysis of data taken from the Collider Detector at Fermilab (CDF), Batavia, Illinois, 60510 USA. The data were studied in order to isolate $B_{s}^{0} \rightarrow J / \psi \eta$ decays.

Research into any fundamental area normally starts with from a general interest within a field of investigation. From studying of other analyses and theoretical motivations a niche is realised. Within the said niche fundamental and original research is possible. Ideally one would like to be able to understand everything, but sadly there simply is not enough time.

W. H. Bell

$30^{\text {th }}$ September 2002 


\section{Author's Declaration}

The work of the CDF collaboration depends on the participation of many people over a long period of time. The author's contribution to the experiment includes taking shifts to monitor the data quality of CDF during Run II, hardware activities during SVXII construction, Run II offline Code browser, and EvtGen integration into the Run II framework.

No portion of the author's work described in this thesis has been submitted in support of an application for another degree or qualification in this, or any other, institute of learning. 


\section{Acknowledgements}

I thank the Physics and Astronomy department at the University of Glasgow for giving me the opportunity to do this research. Thanks to Rick StDenis, and Peter Bussey for correcting drafts of chapters and sections. Thanks to Saverio Dauria for organising the weekly meetings. Thanks to Tony Doyle and David Saxon for their patience during the final stages of writing. Thanks to Ken Smith for encouragement during the last year. Thanks to Petar Maksimovic for suggesting the topic of this thesis. Thanks to Barry Wicklund for many very interesting conversations and for the original VAX fortran code from which much of my analysis code was derived. Thanks to Barry also for his invaluable help reading and checking drafts of the theory chapter. Thanks to Larry Nodulman for bailing me out of hardware and reminding me I had to write a thesis. Thanks to Argonne National Laboratory for providing computational power and funding for two years of this adventure. 


\section{Contents}

1 Theory 17

1.1 Introduction . . . . . . . . . . . . . . . . . 17

1.2 The Standard Model . . . . . . . . . . . . . . . . . . . 18

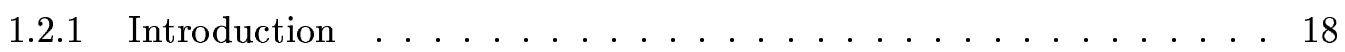

1.2 .2 Properties . . . . . . . . . . . . . . . . . . 19

1.2 .3 The CKM Matrix . . . . . . . . . . . . . . . . 22

1.2 .4 Neutral Meson Mixing . . . . . . . . . . . . . . . 23

1.2.5 CP Violation in Mesons . . . . . . . . . . . . . . 25

1.3 Motivation for this Thesis . . . . . . . . . . . . . . . 27

1.3.1 $\quad B_{s}^{0}$ Width Difference . . . . . . . . . . . . . . . 27

$1.3 .2 \quad$ CP Asymmetry . . . . . . . . . . . . . . . . . 28

1.4 Open Flavour Production $\ldots \ldots \ldots \ldots \ldots$

2 Experimental Apparatus 31

2.1 Introduction . . . . . . . . . . . . . . . . . . 31

2.2 The Tevatron . . . . . . . . . . . . . . . . . . 31

2.3 The Collider Detector at Fermilab . . . . . . . . . . . . . 34

2.3 .1 Introduction . . . . . . . . . . . . . . . . . 34

2.3 .2 Tracking . . . . . . . . . . . . . . . . 35

2.3 .3 Calorimetry ....................... 41

2.3.4 Muon Chambers ................... . . 46

3 Trigger $\quad \mathbf{5 0}$

3.1 Introduction . . . . . . . . . . . . . . . 50

3.2 General Description . . . . . . . . . . . . . . . . 50

3.2 .1 Level $1 \ldots \ldots \ldots \ldots \ldots$

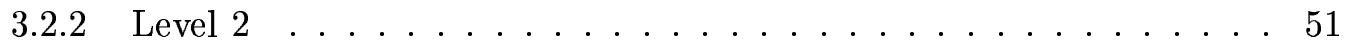

3.2 .3 Level $3 \ldots \ldots \ldots \ldots$. . . . . . . . . . . . . . . 51

$3.3 J / \psi \rightarrow \mu^{+} \mu^{-}$Trigger Path . . . . . . . . . . . . . . . 52

3.3.1 Level 1: Muon . . . . . . . . . . . . . . . . . 52

3.3.2 Central Fast Tracker and Associated Matching . . . . . . . . . . 53

3.3.3 Level 2: Muon . . . . . . . . . . . . . . . . . . . . 55

3.3 .4 Level $3: J / \psi \rightarrow \mu^{+} \mu^{-} \ldots \ldots \ldots \ldots \ldots \ldots \ldots$

3.3 .5 Trigger Summary . . . . . . . . . . . 56 
4 Analysis $\quad \mathbf{5 7}$

4.1 Introduction . . . . . . . . . . . . . . . . 57

4.2 Simulation . . . . . . . . . . . . . . . . . 58

4.2 .1 Introduction . . . . . . . . . . . . . . . 58

$4.2 .2 \quad$ B Meson Production . . . . . . . . . . . . . . . 58

$4.2 .3 \quad B^{+}$and $B_{s}^{0}$ Meson Decays $\ldots \ldots \ldots \ldots$. . . . . . . . 60

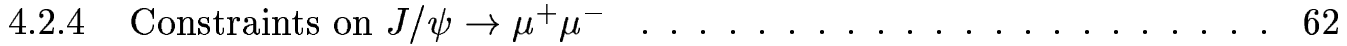

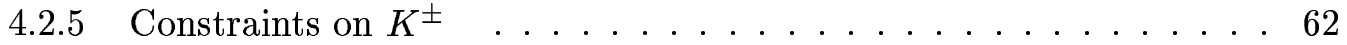

4.2.6 Constraints on $\eta \rightarrow \gamma \gamma \quad \ldots \ldots \ldots \ldots$. . . . . . . . 63

4.3 Analysis of $\mathrm{CDF}$ data . . . . . . . . . . . . . . . 66

4.3 .1 Introduction . . . . . . . . . . . . . . . 66

4.3.2 Selecting a $J / \psi \rightarrow \mu^{+} \mu^{-}$sample . . . . . . . . . . . 66

4.3.3 Finding $B^{ \pm} \rightarrow J / \psi K^{ \pm} \ldots \ldots \ldots \ldots \ldots$

4.3.4 Search for $B_{s}^{0} \rightarrow J / \psi \eta \ldots \ldots \ldots \ldots$

4.4 Efficiencies and Acceptances . . . . . . . . . . . . . . . . . 73

4.4 .1 Introduction . . . . . . . . . . . . . . . . 73

4.4.2 $K^{ \pm}$Reconstruction Efficiency . . . . . . . . . . . . 74

4.4.3 CES Reconstruction Efficiency . . . . . . . . . . . . . . 74

4.5 Detailed Simulation . . . . . . . . . . . . . . . . 85

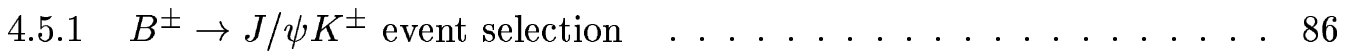

4.5.2 Modelling photons in the calorimeters . . . . . . . . . 86

4.5.3 Generating $B_{s}^{0} \rightarrow J / \psi \eta$ events . . . . . . . . . . . . 88

4.5.4 CES purity and rate of ambiguity $\ldots \ldots \ldots . \ldots . \ldots 89$

5 Statistical and Systematic Errors $\quad 91$

5.1 Introduction . . . . . . . . . . . . . . . . . 91

5.2 External Errors . . . . . . . . . . . . . . . . . . . . 91

5.3 Monte Carlo Ratio . . . . . . . . . . . . . . . . . . . . . 92

5.3 .1 Introduction . . . . . . . . . . . . . . . . 92

5.3 .2 B Meson Production . . . . . . . . . . . . . . . . . . 92

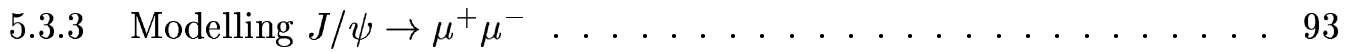

$5.3 .4 \quad K^{ \pm}$Reconstruction Efficiency . . . . . . . . . . . . 94

5.3.5 CES Reconstruction Efficiency . . . . . . . . . . . . . . 95

5.4 Data Analysis . . . . . . . . . . . . . . . . . . . . . 95

$5.4 .1 \quad B^{ \pm} \rightarrow J / \psi K^{ \pm} \ldots \ldots \ldots \ldots \ldots \ldots \ldots$

5.4 .2 Lifetime Constraints . . . . . . . . . . . . . . . . 96

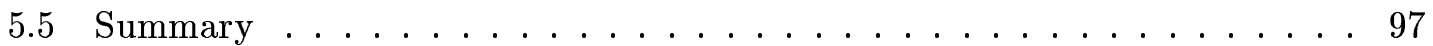

6 Results $\quad 99$

6.1 Introduction . . . . . . . . . . . . . . . . . . 99

$6.2 \quad B^{ \pm} \rightarrow J / \psi K^{ \pm} \ldots \ldots \ldots \ldots \ldots \ldots \ldots \ldots \ldots \ldots$

6.3 Branching Ratio $\mathcal{B}\left(B_{s}^{0} \rightarrow J / \psi \eta\right) \ldots \ldots \ldots \ldots \ldots$

6.3.1 Events Predicted . . . . . . . . . . . . . . . 99

6.3.2 Events Observed . . . . . . . . . . . . . . . . . 100

$6.3 .390 \%$ Confidence Limit . . . . . . . . . . . . . . . . 100

6.3.4 Number Ratio $N\left(J / \psi K^{+}\right) / N(J / \psi \eta) \quad \ldots \ldots \ldots$

$\begin{array}{lll}7 & \text { Summary, Conclusions and Prospects } & 104\end{array}$ 
$\begin{array}{lr}\text { A Modelling Muon Acceptances } & \mathbf{1 0 6}\end{array}$

A.1 Primary Vertex Generation . . . . . . . . . . . . . . . . . . 106

A.2 CMU and CMX Geometrical Acceptance . . . . . . . . . . . . . 107

A.2.1 CMU Acceptance . . . . . . . . . . . . . . . . . . 108

A.2.2 CMX Acceptance . . . . . . . . . . . . . . . 108

A.3 Level 1 Muon Trigger Acceptance . . . . . . . . . . . . . . . . . . 110

A.4 Level 2 Muon Trigger Acceptances . . . . . . . . . . . . . . . . . 111

B Proportional Strip Chamber Clustering $\quad 113$

C A study of photon acceptances $\mathbf{1 1 5}$

C.1 Introduction . . . . . . . . . . . . . . . . . . . . . . 115

C.2 Previous Publications . . . . . . . . . . . . . . . . 115

C.3 Kinematics and Acceptance . . . . . . . . . . . . . . . . 116

C.4 Calorimeter Performance Effects . . . . . . . . . . . . . . . . 117

C.4.1 Calorimeter Energy Resolution . . . . . . . . . . . . . . 117

C.4.2 CES Reconstruction Efficiency . . . . . . . . . . . 117

C.5 Diphoton background . . . . . . . . . . . . . . 118

D Conversion Electron Selection Cuts $\quad 121$

D.1 Introduction . . . . . . . . . . . . . . . . . . . . . 121

D.2 Pair Selection . . . . . . . . . . . . . . . . . 121

D.3 Identified Electron Track . . . . . . . . . . . . . . . . . 121

D.4 Conversion Partner Electron . . . . . . . . . . . . . . . . . . . 122

$\begin{array}{lr}\text { E Tracking Parameters } & 125\end{array}$ 


\section{List of Figures}

1.1 Bottom quark decay into a strange quark and a $\mathrm{J} / \psi$ meson. From left to right the magnitudes of coupling at the two $W^{+}$vertices are given by $V_{c b}^{*}$

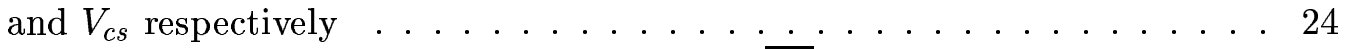

1.2 Box diagrams illustrating a $B_{s}^{0}$ mixing into a $\overline{B_{s}^{0}}$ meson $\ldots \ldots \ldots \ldots . . \ldots 24$

1.3 Tree level heavy quark flavour creation subprocesses. . . . . . . . . . . 29

1.4 Tree level heavy quark flavour excitation subprocesses. . . . . . . . . . . 29

2.1 The Tevatron with pre-accelerators $\ldots \ldots \ldots \ldots \ldots$

2.2 The CDF detector during the Run I period of 1992 to $1996 \ldots \ldots$

2.3 The CDF detector during the Run I period of 1992 to 1996. The illustration shows one quarter of the detector. The complete detector was symmetric

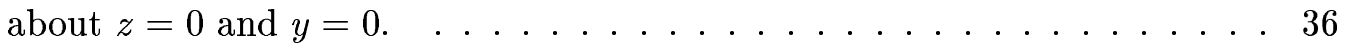

2.4 A typical Silicon Vertex detector ladder. . . . . . . . . . . . . 37

2.5 A schematic diagram of one of two Silicon Vertex detector barrels showing all four layers of silicon together with aluminium bulk-heads. The two barrels when coupled together formed a $60 \mathrm{~cm}$ active region. . . . . . . 38

2.6 The Vertex Time Projection Chamber, showing sense wires and octants [38]. 40

2.7 The Central Tracking Chamber end plate. Regions superlayer wires were connected are shown. The Axial superlayers are composed of less wires . . 41

2.8 A typical Central Electromagnetic Calorimeter module. . . . . . . . . . . . 44

2.9 A plot summarising central muon coverage for the run period 1992-96. Plain gray patches are un-instrumented regions. . . . . . . . . . 47

2.10 The end of a single muon module. Three of these were bolted together and attached to the back of each hadronic calorimeter tower. . . . . . . . 48

3.1 A high momentum track crossing one layer of sense wires within an axial CTC superlayer. The error bars roughly correspond to the timing windows within which prompt and delayed hits could be accepted. . . . . . . . . 54

4.1 Renormalised NLO QCD cross-sections for $B^{ \pm}$production compared with CDF data[18]. B mesons were generated using Peterson fragmentation. The theoretical curves have been renormalised in an attempt to fit the data. . . 59

4.2 The Peterson fragmentation function for $\epsilon_{Q}=0.006$, the value used for $b$ quark decays at CDF. . . . . . . . . . . . . . . 61

4.3 A comparison of $M(J / \psi \eta)-M\left(B_{s}^{0}\right)$ assuming the width is only dependent on the electromagnetic calorimeter resolution. Left and right plots correspond to the corrected (equation 4.3) and uncorrected mass distributions. . 64

4.4 Decay of $B_{s}^{0} \rightarrow J / \psi \eta$ or $B_{s}^{0} \rightarrow J / \psi \phi \ldots \ldots \ldots \ldots \ldots$ 
4.5 The distribution of muon pair primary vertices with respect to each other, where $\Delta z_{0}=z 1_{0}-z 2_{0}$. The selected region is marked with vertical lines and shaded yellow.

4.6 The invariant mass distribution for two opposite sign muons both passing matching constraints between track and muon stub. Left without SVX requirements, Right requiring three or more SVX hits for each muon

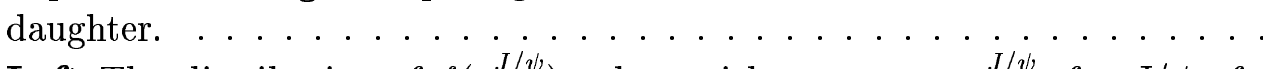

4.7 Left The distribution of $f\left(p_{t}^{J / \psi}\right)$ values with respect to $p_{t}^{J / \psi}$, for $J / \psi$ of $B_{s}^{0} \rightarrow J / \psi \eta$ decays passing all constraints. Right Mean behaviour of $f\left(p_{t}^{J / \psi}\right)$ with respect to $p_{t}^{J / \psi}$. The error bars shown were calculated from the standard deviation of each vertical bin slice. The dotted line shows the kinematic limit. Bottom The $p_{t}^{J / \psi}$ spectrum of these events. . . . . . 73

4.8 CTC Tracking efficiency vs $p_{t}$ for early and late runs, taken from [40]. Combining all runs the single track efficiency for transverse momenta above $0.4 \mathrm{GeV} / \mathrm{c}$ and exit radii greater than $110 \mathrm{~cm}$, was found to be $92.8 \pm 2.0 \%$.

4.9 Definition of the tangent point between two tracks. . . . . . . . 76

4.10 A histogram of the radial displacement of $e^{+} e^{-}$conversion vertices from the origin. Conversion electrons produced by interaction with the CTC can and the outermost layer of the inner detector are shown in yellow. . . . . 77

4.11 The efficiency of finding a CES cluster within a tower into which a conversion electron partner track projected. . . . . . . . . . . 80

4.12 The distribution of $e^{+} e^{-}$conversion vertices along the beam axis where each conversion pair passed all of the constraints described in section 4.4.3.3 81

4.13 The distribution of radiation lengths seen by a conversion partner electron crossing the solenoid and electromagnetic calorimeter material in front of the CES chamber ...................... 82

4.14 Longitudinal shower progression using fit parameters given in table 4.9 and assuming the CES to be at a constant depth of six radiation lengths. . . . 8

4.15 The reconstruction efficiency for an electromagnetic shower within the CES with respect to the predicted energy measurement within the CES. The error bars shown were calculated from the binomial error given in equation $4.10 . \ldots \ldots \ldots \ldots \ldots$. . . . . . . . . . . . . . . . 85

4.16 Selected $B^{ \pm} \rightarrow J / \psi K^{ \pm}$events, where: $\Delta M\left(B^{ \pm}\right)=m_{B^{ \pm}}^{P D G}-m_{B^{ \pm}}^{\text {meas }} \ldots . \quad 86$

4.17 A figure illustrating a possible CES ambiguity scenario. The real photon pair shower cross-section at the CES depth is shown in grey. . . . . . . 89

4.18 Left A histogram of the fitted shower centroid position from CES clustering minus the original position of the particle. Right A histogram of the fitted shower centroid position from CES clustering minus the off diagonal 'ghost' cluster position. . . . . . . . . . . . . . . .

5.1 Mass distributions for $B_{s}^{0} \rightarrow J / \psi \eta$ candidates: Left $M(\gamma \gamma)$ within $120 \mathrm{MeV}$ of the world average $M(\eta)$, and Right $120 \mathrm{MeV}$ either side of the $M(\gamma \gamma)$ central window. Both plots show the mass distributions with the three limits of $f\left(p_{t}^{J / \psi}\right)$ fit parameters. . . . . . . . . . . . .

6.1 The distribution of $c t_{\text {true }}$ for the fully reconstructed $B^{ \pm} \rightarrow J / \psi K^{ \pm}$sample. The data shown passed all of the constraints except the requirement made on $c t_{\text {true }}$ and are within $60 \mathrm{MeV}$ of the world average $B^{ \pm}$mass. . . . . 100 
6.2 The reconstructed $B^{ \pm}$mass distribution from fully reconstructed $B^{ \pm} \rightarrow$ $J / \psi K^{ \pm}$events. From the fit shown there are $490 \pm 23$ events above background. . . . . . . . . . . . . . . . . . . 101

6.3 The reconstructed $B_{s}^{0}$ mass distribution from $B_{s}^{0} \rightarrow J / \psi \eta$ candidates. The curve is the Monte Carlo distribution renomalised for the predicted number of $B_{s}^{0} \rightarrow J / \psi \eta$ events. . . . . . . . . . . . . . . 102

6.4 An illustration of a $90 \%$ Gaussian confidence limit. . . . . . . . . . . 102

A.1 Primary vertex distribution after basic muon constraints: left without SVX $\left({ }^{\prime}\right)$ constraints, right with three or more hits required on each muon leg.107

A.2 Left Primary vertex acceptance after geometric acceptance, level 1 and level 2 trigger simulations have been applied. (Each event contained two muons from a $B_{s}^{0} \rightarrow J / \psi \eta$ decay. No $p_{t}$ cut on the $B_{s}^{0}$.); Right The histogram used to generate primary vertices with associated one sigma errors. . . . 108

A.3 A muon track in the $r-z$ plane, intersecting the CMU axis. . . . . . . 109

A.4 Perpendicular distance from the CMX axis to the primary vertex in the $r-z$ plane . . . . . . . . . . . . . . . 109

A.5 An illustration of $\delta L$ in the $r-z$ plane $\ldots \ldots \ldots \ldots \ldots \ldots$

A.6 Level 1 trigger efficiencies as a function of transverse momentum $\left(p_{t}\right)$ : left CMU, right CMX. The errors either side of the mean were extracted from varying the fit parameters within one sigma of each associated error. . . . 111

A.7 Level 2 trigger efficiencies for a CFT match as a function of transverse momentum $\left(p_{t}\right)$ : left CMU, right CMX. The errors either side of the mean were extracted from varying the fit parameters within one sigma of each associated error. Azimuthal and pseudorapidity effects have been taken at their mean values. . . . . . . . . . . . . . . . . . . . . 11

C.1 Photon daughters from $B_{s}^{0} \rightarrow J / \psi \eta$ produced using NLO QCD, MRST99 (central gluon), and Peterson fragmentation. $J / \psi$ trigger constraints have been made. . . . . . . . . . . . . . . . . . . 116

C.2 Acceptance of $B_{s}^{0} \rightarrow J / \psi \eta$ where $\eta \rightarrow \gamma \gamma$, as a function of the calorimeter energy cut made on both photons. . . . . . . . . . . . . . . . 117

C.3 left CEM calorimeter energy resolution as a function of $E_{t}$. The calorimeter resolution $13.5 \% / \sqrt{E}$ was taken from [9]. right $\Gamma\left(B_{s}^{0}\right)$ as a function of the $E_{t}$ cut, assuming the width is only due to the electromagnetic calorimeter resolution. . . . . . . . . . . . . . . . . . 11

C.4 $M(\gamma \gamma)$ mass distribution for different $E_{t}$ constraints. From the top left to the bottom right, the distributions were made from $J / \psi$ stream B data requiring $E_{t}$ to be greater than $0.4,0.6,0.8$ and $1.0 \mathrm{GeV}$ respectively. . . 119

C.5 Summary plots showing the behaviour of the $\pi^{0}$ peak within the $M(\gamma \gamma)$ mass distribution. left Signal/background for the $\pi^{0}$ peak as a function of the $E_{t}$ requirement. right The number of background events under the $\pi^{0}$

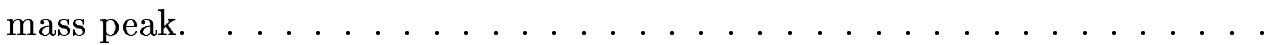

D.1 Left: the distribution of tangent point values, showing the selected region in yellow. Right: the distribution of $\Delta \cot (\theta)$ values, showing the selected region in yellow. . . . . . . . . . . . . . . . .

D.2 Left: CES strip fit $\chi^{2}$, showing the selected region in yellow. Right: CES wire fit $\chi^{2}$, showing the selected region in yellow. 
D.3 Hadronic/Electromagnetic energy for the identified electron. The selected region is shown in yellow. . . . . . . . . . . . . . . 123

D.4 Left: $z_{f i t}-z_{\text {hel }}$, showing the selected region in yellow. Right: $x_{f i t}-x_{\text {hel }}$, showing the selected region in yellow. . . . . . . . . . . . . 123

D.5 The $p_{t}$ spectrum of the conversion partner. Events within the selected region are shown in yellow. . . . . . . . . . . . . . . . 124

D.6 Background electromagnetic energy within the conversion partners CEM module. (This plot was formed by summing the energy within the tower and then removing the energy of the conversion electron partner.) Events that fall within the selected region are shown in yellow. . . . . . . . . . 124

E.1 A helical track within two, two dimensional planes: where $\rho$ is the radius of the helix and $D$ is the impact parameter. . . . . . . . . . 125 


\section{List of Tables}

1.1 Properties of the fundamental constituents of matter. The quark masses quoted are the bare quark masses. All masses were taken from[33]. The confidence limits for the neutrino mass are $95 \%$ with the exception of $\nu_{\mu}$

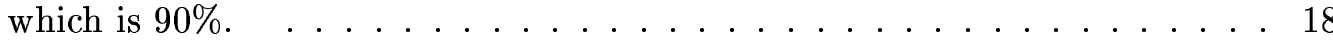

1.2 Forces observed between matter particles. (The Graviton has not been experimentally observed.) . . . . . . . . . . . . . . 19

2.1 Comparison of SVX and SVX(') . . . . . . . . . . . 39

2.2 Number of radiation lengths for central electromagnetic calorimeter stack up and solenoid coil. . . . . . . . . . . . . . 43

3.1 Summary of CFT $p_{t}$ binning. The table was re-optimised at the beginning of Run IB . . . . . . . . . . . . . . . . . . . . 5 54

3.2 Level $3 J / \psi \rightarrow \mu^{+} \mu^{-}$trigger constraints during the Run I operational period. ......................... 56

3.3 A summary of the main $J / \psi$ level 2 triggers during the Run Ib running period taken from $[49] . \ldots \ldots \ldots \ldots 6$

4.1 Constraints made on the generated $K^{ \pm}$particle from the decay $B^{ \pm} \rightarrow$

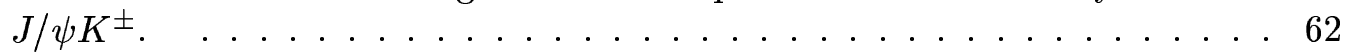

4.2 Meson Particle Properties from the Particle Data Book [33]. . . . . . . . 65

$4.3 \mathrm{~J} / \psi$ selection criterion made on the $J / \psi$ stream B sample . . . . . . . . 69

4.4 A summary of the constraints made on the kaon candidate and the reconstructed $B^{ \pm}$meson. . . . . . . . . . . . . . . 71

4.5 A summary of the constraints made on the $\eta$ candidate and the $B_{s}^{0}$ candidate. . . . . . . . . . . . . . . . . 72

4.6 Selection criterion for conversion pairs . . . . . . . . . . . . 76

4.7 Constraints applied to the identified electron leg of a conversion pair . . . 78

4.8 Cuts applied to the un-biased conversion partner, where $\left(\sum E M\right)$ implies the total electromagnetic energy within the CEM module. . . . . . . 79

4.9 A table of longitudinal shower fit parameters from [35]. . . . . . . . . 83

4.10 CES efficiency curve fit parameters, where $y=p_{3}\left(1-\exp \left(p_{2}-p_{1} x\right)\right) \ldots 84$

4.11 Constraints applied to the conversion partner to isolate a sample of low energy CES shower profiles. . . . . . . . . . . . 88

5.1 Errors from parameters not measured during this analysis, but incorporated to form the confidence limit . . . . . . . . . . . . . . . 92 
5.2 Input value ranges used during $\mathrm{B}$ meson production: where $m_{b}$ is the $\mathrm{b}$ quark mass, $\mu$ is the renormalisation and factorisation scale and $\epsilon_{Q}$ is the tuning parameter for Peterson fragmentation. . . . . . . . . . 93

5.3 Systematic errors within B meson production . . . . . . . . . . . 93

5.4 Systematic errors within primary vertex generation. . . . . . . . . . . . . 93

5.5 Systematic errors within the level $1 J / \psi \rightarrow \mu^{+} \mu^{-}$trigger acceptance curve fits. . . . . . . . . . . . . . . . . . 94

5.6 Systematic errors within the level $2 J / \psi \rightarrow \mu^{+} \mu^{-}$trigger acceptance curve fits. . . . . . . . . . . . . . . . . 94

5.7 Systematic errors from the longitudinal shower fit parameters and CES efficiency fit to conversion electron data. . . . . . . . . . . . 95

5.8 Systematic errors from the uncertainty in the $f\left(p_{t}^{J / \psi}\right)$ distribution. $\quad \ldots .96$

5.9 A summary table of all uncertainties. ( $\dagger$ All of these errors were calculated by varying the associated parameter within the Monte Carlo. $\ddagger$ This error was propagated into the final result by simply picking the highest upper $90 \%$ C.L. i.e. lower limit.) . . . . . . . . . . . . . . 98

6.1 A table of number ratios $N\left(J / \psi K^{+}\right) / N(J / \psi \eta)$ with and without the detector related acceptance factors. (Corrected $N\left(J / \psi K^{+}\right) / N(J / \psi \eta) \Longrightarrow$ $\left.M C(J / \psi \eta) / M C\left(J / \psi K^{+}\right) \times N\left(J / \psi K^{+}\right) / N(J / \psi \eta)\right) \ldots \ldots \ldots$ 


\section{Chapter 1}

\section{Theory}

\section{$1.1 \quad$ Introduction}

This chapter briefly describes the origins of particle physics, leading to a summary of the different parameters within the current model of forces and their interaction with elementary particles. From the general theory attention turns to the interactions fundamental to bottom quark decays. Finally a discussion of the properties of the $B_{s}^{0} \rightarrow J / \psi \eta$ and the possible new physics that can be accomplished if a large sample of these decays can be isolated.

The theoretical concepts are summarised within; for more information, good introductory material can be found in [32], a phenomenological overview is provided by [10] and [37] provides a more detailed theoretical introduction.

For simplicity, Natural Units are used within this chapter and the rest of the thesis. These units are defined by setting the constants

$$
\hbar=c=1
$$

Quantities given in SI units can be obtained by inserting the correct powers of $\hbar$ and $c$ via a dimensional analysis. 


\subsection{The Standard Model}

\subsubsection{Introduction}

The Standard Model embodies knowledge of the strong and electroweak interactions. All attempts to find naked quarks have failed and the number of quarks and leptons now seems to be stable at 3 generations. The bare quark masses remain free parameters.

The Standard Model describes the current theory of fundamental particles and their interaction with each other. Matter is made up of quarks and leptons, both of which are fermions. A fermion is a particle that has odd half-integer $(1 / 2,3 / 2)$ intrinsic angular momentum. This intrinsic angular momentum is normally referred to as spin and is measured in units of $\hbar$. Each quark and lepton has spin one half. Both particles are said to be fundamental because there is no experimental evidence for quark or lepton compositeness, such as excited states or form factors. Some of the properties of the fundamental fermions are summarised in table 1.1.

\begin{tabular}{|c|c|c|c|c|c|}
\hline \hline \multicolumn{3}{|c|}{ Quarks } & \multicolumn{3}{c|}{ Leptons } \\
\hline Flavour & Mass & Charge & Flavour & Mass & Charge \\
\hline $\mathrm{u}$ & $3 \pm 2 \mathrm{MeV}$ & $\frac{2}{3} e$ & $\nu_{e}$ & $<3 \mathrm{eV}$ & 0 \\
$\mathrm{~d}$ & $6 \pm 3 \mathrm{MeV}$ & $-\frac{1}{3} e$ & $e$ & $0.510998902 \pm 2.1 \times 10^{-8} \mathrm{MeV}$ & $-e$ \\
$\mathrm{c}$ & $1.25 \pm 0.1 \mathrm{GeV}$ & $\frac{2}{3} e$ & $\nu_{\mu}$ & $<0.19 \mathrm{MeV}$ & 0 \\
$\mathrm{~s}$ & $122 \pm 48 \mathrm{MeV}$ & $-\frac{1}{3} e$ & $\mu$ & $105.6583568 \pm 5.2 \times 10^{-6} \mathrm{MeV}$ & $-e$ \\
$\mathrm{t}$ & $174.3 \pm 5.1 \mathrm{GeV}$ & $\frac{2}{3} e$ & $\nu_{\tau}$ & $<18.2 \mathrm{MeV}$ & 0 \\
$\mathrm{~b}$ & $4.2 \pm 0.2 \mathrm{GeV}$ & $-\frac{1}{3} e$ & $\tau$ & $1776.99 \pm 0.29 \mathrm{MeV}$ & $-e$ \\
\hline \hline
\end{tabular}

Table 1.1: Properties of the fundamental constituents of matter. The quark masses quoted are the bare quark masses. All masses were taken from[33]. The confidence limits for the neutrino mass are $95 \%$ with the exception of $\nu_{\mu}$ which is $90 \%$.

Unlike leptons quarks have an additional quantum number called colour. Each flavour of quark can have one of three colour charges. Naked colour charge and free quarks have not been experimentally observed. Quarks are normally confined inside particles called hadrons. These hadrons contain two subgroups of particles: baryons, composed of three quarks and mesons composed of a quark and an anti-quark. Both of these are said to 
be colourless because the baryonic quarks are red, green and blue, where as the quarks contained within a meson are colour and anti-colour. Within the Universe most of the visible matter is composed of protons and neutrons both of which are baryons.

Matter interacts with other matter by one of four forces: the Strong force, the Weak force, Electromagnetism, and Gravity. Each of these forces is mediated by the exchange of integer spin particles called Bosons. The properties of the fundamental forces are given in table 1.2 .

\begin{tabular}{|c|c|c|c|c|}
\hline \hline Interaction & Effective Coupling & Boson & Range $(\mathrm{m})$ & Mean Time (s) \\
\hline Gravitation & $10^{-39}$ & graviton & $\infty$ & - \\
Electromagnetic & $1 / 137$ & photon & $\infty$ & $10^{-20}$ \\
Weak force & $10^{-5}$ & $\mathrm{~W}^{ \pm}, \mathrm{Z}^{0}$ & $10^{-18}$ & $10^{-10}$ \\
Strong force & 1 & gluons & $<10^{-15}$ & $10^{-23}$ \\
\hline \hline
\end{tabular}

Table 1.2: Forces observed between matter particles. (The Graviton has not been experimentally observed.)

Not all fundamental particles experience all of the fundamental forces. Leptons do not carry a colour charge and therefore are un-affected by the strong force. Neutrinos do not have an electric charge and therefore do not take part in any electromagnetic interactions. Everything is subject to gravitation but it is so weak with respect to the other forces, that it is commonly ignored at small scales.

The strength of each force is determined by its effective coupling. The effective coupling is not a constant and is itself a function of energy. Values of the effective couplings are given in table 1.2 for normal bound matter.

\subsubsection{Properties}

The Standard Model is a quantum field theory based on the gauge symmetry $S U(3)_{c} \times$ $S U(2)_{L} \times U(1)_{Y}$. Within this gauge invariant theory of strong and electroweak forces $S U(3)_{c}$ is a symmetry group containing $3 \times 3$ hermitian matrix operators. These transformations can change the colour charge hence the subscript $c$ given to the group. This model requires the presence of eight massless gauge bosons referred to as gluons. Gluons are massless, electrically neutral and carry a colour quantum number. Since gluons 
themselves carry colour charge, they interact with themselves as well as quarks.

$S U(2)_{L} \times U(1)_{Y}$ represents the Glashow-Salam-Weinberg theory of electroweak interactions. The subscripts $L_{L}$ and $Y$ refer to isospin coupling only to the left-handed particles and weak hypercharge respectively, whereas $U(1)$ implies a group of unitary transformations in one dimension and $S U(2)$ is a symmetry group containing $2 \times 2$ hermitian matrix operators. This theory implies four gauge bosons: an isotriplet $\left(\left(W_{\mu}^{i}\right)\right.$ where $\left.i=1,3\right)$ within $S U(2)_{L}$, and a singlet $B_{\mu}$ within $U(1)_{Y}$. The mixing between the neutral current components corresponding to the $Z^{0}$ and $\gamma$ gauge bosons, is parameterised by the electroweak mixing angle. Electromagnetic interactions are therefore a subgroup of $S U(2)_{L} \times U(1)_{Y}$.

V-A Theory of Charged Weak Interactions Before introducing the form of the current theory of weak interactions it is necessary to look at $V-A$ theories in general. Parity violation was observed within $K^{+}$decays namely $K^{+} \rightarrow \pi^{+} \pi^{0}$ and $K^{+} \rightarrow \pi^{+} \pi^{+} \pi^{-}$, together with ${ }^{60} \mathrm{Co}$ nuclei decay. It was from the observations of the ${ }^{60} \mathrm{Co}$ nuclei that $V-A$ as a theoretical model was born. The experimental observations gave rise to the conclusion that the charged weak currents responsible for the decays within the ${ }^{60} \mathrm{Co}$ nuclei always produce left-handed electrons and right-handed anti-neutrinos. The lack of any righthanded neutrinos in processes mediated by the charged weak interactions is a manifest signal of parity violation. Furthermore this is evidence of maximal parity violation which leads to the conclusion that the charged weak current can be described by the vector minus axial form or $V-A$ :

$$
J_{\mu} \sim V_{\mu}-A_{\mu}
$$

where $J_{\mu}$ is the charged weak current, $V_{\mu}$ is the vector current, and $A_{\mu}$ is the axial current.

The parity violating nature of $V-A$ currents can be illustrated by considering the product transformations of

$$
V^{\mu}=\bar{\psi} \gamma^{\mu} \psi, A^{\mu}=\bar{\psi} \gamma^{\mu} \gamma^{5} \psi
$$

It can be shown that under the parity operator:

$$
V_{\mu} V^{\mu} \rightarrow V_{\mu} V^{\mu}, A_{\mu} A^{\mu} \rightarrow A_{\mu} A^{\mu}, A_{\mu} V^{\mu} \rightarrow-A_{\mu} V^{\mu}
$$

The charged current enters the Lagrangian as $J_{\mu} J^{\mu}$. Therefore from the previous product transformations $J_{\mu} \sim V_{\mu}-A_{\mu}$ is maximally violating:

$$
J_{\mu} J^{\mu} \sim\left(V_{\mu}-A_{\mu}\right)\left(V^{\mu}-A^{\mu}\right),
$$


under the parity operator,

$$
\left(V_{\mu}-A_{\mu}\right)\left(V^{\mu}-A^{\mu}\right) \rightarrow\left(V_{\mu}+A_{\mu}\right)\left(V^{\mu}+A^{\mu}\right)
$$

The charge current can be re-written in terms of the field components.

$$
J_{\mu} \sim V_{\mu}-A_{\mu}=\overline{\nu_{e}} \gamma_{\mu}\left(1-\gamma_{5}\right) e=2 \overline{\left(\nu_{e}\right)_{L}} \gamma_{\mu} e_{L}
$$

This demonstrates that within the $V-A$ theoretic framework, charged weak interactions only couple to left-handed fermions or right-handed anti-fermions.

Feynman and Gell-Mann Feynman and Gell-Mann formulated the $V-A$ theory [28] to explain parity violation by weak interactions. The $V-A$ theory Lagrangian for the first two generations of quarks and leptons is given by:

$$
\mathcal{L}_{V-A}=-\frac{G_{F}}{\sqrt{2}} J_{\mu}^{c c}(x) J^{c c \mu}(x)
$$

where the charged current is given by

$$
J_{\mu}^{c c}=\overline{\nu_{e}} \gamma_{\mu}\left(1-\gamma_{5}\right) e+\overline{\nu_{\mu}} \gamma_{\mu}\left(1-\gamma_{5}\right) \mu+\bar{u} \gamma_{\mu}\left(1-\gamma_{5}\right) d
$$

$G_{F}$ is the Fermi constant which can be measured from the $\mu$ lifetime, $u$ is the weak eigenstate of the up quark and $d$ is the weak eigenstate of the down quark. These are different from the mass states and are related to the mass states via the Cabibbo angle $\theta_{c}$,

$$
d=d_{m} \cos \theta_{c}+s_{m} \sin \theta_{c}
$$

where $d_{m}$ and $s_{m}$ are the mass eigenstates of the down and strange quark respectively. This theory formulated by Feynman and Gell-Mann describes the observed weak interactions but does suffer from some problems. The V-A theory violates unitarity at high energies and is a non-renormalisable theory.

Glashow-Salam-Weinberg Glashow Salam and Weinberg used the ideas from Feynman and Gell-Mann together with the Intermediate Vector Boson (IVB) theory [43][31] to assemble the $S U(2)_{L} \times U(1)_{Y}$ gauge theory. The IVB theory incorporated an interesting idea of massive vector bosons: two charged $W^{ \pm}$and one neutral $Z^{0}$. It also had its problems containing both $V+A$ and $V-A$ charged current components, nonrenormalisability and violation of the unitarity bound at high energies. 
The $S U(2)_{L} \times U(1)_{Y}$ theory solves all of these problems. By constructing a gauge theory to unify the electromagnetic and weak forces the force carriers from IVB theory became the gauge bosons. Beyond this gauge coupling was included such that vector bosons could self-interact. This repaired the divergences observed within the previous theories and cancelled the un-wanted high energy behaviour.

\subsubsection{The CKM Matrix}

Before introducing the specific concepts of b-physics it is necessary to look at electroweak interaction between quarks. This discussion can be found in more detail in [54].

Written as a Lagrangian density the unified model of electroweak interactions for quarks is:

$$
\mathcal{L}=\mathcal{L}_{q}^{0}+\mathcal{L}_{q}^{H}+\mathcal{L}_{q}^{c c}+\mathcal{L}_{q}^{n c}
$$

where $\mathcal{L}_{q}^{0}$ is the kinetic (or Dirac) Lagrangian, $\mathcal{L}_{q}^{H}$ represents the couplings of the quarks to the Higgs boson, $\mathcal{L}_{q}^{c c}$ refers to the charged current coupling and $\mathcal{L}_{q}^{n c}$ refers to the neutral current coupling. The charged current Lagrangian, $\mathcal{L}_{q}^{c c}$, describes the interaction between quarks and $W$ bosons, written simply as:

$$
\mathcal{L}_{q}^{c c}=-\frac{g}{\sqrt{2}}\left(J_{\mu}^{c c \dagger} W^{\mu}+J_{\mu}^{c c} W^{\mu \dagger}\right)
$$

where $g$ is the coupling constant, $J_{\mu}^{c c}$ is the charged current, and $W^{\mu}$ is the W-field.

The charged current $J_{\mu}^{c c}$, which couples to the $W^{ \pm}$can be expanded as [41]:

$$
J_{\mu}^{c c}=\left(\begin{array}{lll}
\bar{u} & \bar{c} & \bar{t}
\end{array}\right)_{L} \gamma_{\mu} V_{C K M}\left(\begin{array}{l}
d \\
s \\
b
\end{array}\right)_{L}
$$

where $V_{C K M}$ is a $(3 \times 3)$ unitary matrix in flavour space, and the elements are:

$$
V_{C K M} \equiv\left(\begin{array}{ccc}
V_{u d} & V_{u s} & V_{u b} \\
V_{c d} & V_{c s} & V_{c b} \\
V_{t d} & V_{t s} & V_{t b}
\end{array}\right)
$$

There are no restrictions on $V_{C K M}$ except the requirement of unitarity. This unitary nature implies that the hermitian conjugate be equal to the inverse: $V_{C K M}^{\dagger} V_{C K M}=1$. More explicitly, unitarity implies any pair of rows, or any pair of columns are orthogonal. These conditions can be written as: 


$$
\begin{array}{ll}
\sum_{i}^{\{u, c, t\}} V_{i d} V_{i s}^{*}=0 & \sum_{j}^{\{d, s, b\}} V_{u j} V_{c j}^{*}=0 \\
\sum_{i}^{\{u, c, t\}} V_{i s} V_{i b}^{*}=0 & \sum_{j}^{\{d, s, b\}} V_{c j} V_{t j}^{*}=0 \\
\sum_{i}^{\{u, c, t\}} V_{i d} V_{i b}^{*}=0 & \sum_{j}^{\{d, s, b\}} V_{u j} V_{t j}^{*}=0
\end{array}
$$

The orthogonality conditions can be drawn as six triangles in the complex plane. Not all of the angles and sides are independent. It has been shown in [52], that only four of the eighteen angles contained in the six triangles are independent. Four useful angles, two large angles $\beta, \gamma$, and two small angles $\beta_{s}$ and $\beta_{k}$ :

$$
\begin{array}{ll}
\beta=\arg \left(-\frac{V_{c d} V_{c b}^{*}}{V_{t d} V_{t b}^{*}}\right) & \gamma=\arg \left(-\frac{V_{u d} V_{u b}^{*}}{V_{c d} V_{c b}^{*}}\right) \\
\beta_{s}=\arg \left(-\frac{V_{t s} V_{b b}^{*}}{V_{c s} V_{c b}^{*}}\right) & \beta_{k}=\arg \left(-\frac{V_{c s} V_{c d}^{*}}{V_{u s} V_{u d}^{*}}\right)
\end{array}
$$

are commonly used parameterisations of the CKM matrix elements. The angle $\beta_{s}$ will be discussed later, while the others are included for completeness.

The matrix elements of $V_{C K M}$ are determined by the relative strength of coupling at each two quark $W$ vertex combinations. Examples of two quark $W$ vertices are given in figure 1.1, where the $V_{c b}$ and $V_{c s}$ matrix elements are involved. At a $90 \%$ confidence limit, the current world average moduli of the $V_{C K M}$ matrix elements are [33]:

$$
\left(\begin{array}{cc}
\left|V_{i j}\right| & \rightarrow \\
\downarrow & \searrow
\end{array}\right)=\left(\begin{array}{ccc}
0.97495 \pm 0.00075 & 0.2225 \pm 0.0035 & 0.0035 \pm 0.0015 \\
0.222 \pm 0.003 & 0.97415 \pm 0.00075 & 0.04 \pm 0.003 \\
0.009 \pm 0.005 & 0.039 \pm 0.004 & 0.99915 \pm 0.00015
\end{array}\right)
$$

There are many parameterisations relating the values of the different CKM matrix elements. One useful parameterisation is the Wolfenstein parameterisation, given in [57]. In this parameterisation $\lambda \equiv \sin \theta_{c}$, where $\theta_{c}$ is the Cabibbo mixing angle.

$$
V_{C K M} \approx\left(\begin{array}{ccc}
1-\frac{1}{2} \lambda^{2} & \lambda & \lambda^{3} A(\rho-i \eta) \\
-\lambda & 1-\frac{1}{2} \lambda^{2} & \lambda^{2} A \\
\lambda^{3} A(1-\rho-i \eta) & -\lambda^{2} A & 1
\end{array}\right)
$$

This is an approximation and is only satisfies unitarity to order $\lambda^{3}$.

\subsubsection{Neutral Meson Mixing}

Mixing is the process where a neutral meson such as a $K^{0}, B_{d}^{0}$, or $B_{s}^{0}$ oscillates from its matter state into its anti-matter state and vica versa. This is a second-order weak interaction illustrated in figure 1.2. The frequency of the mixing oscillation is determined 


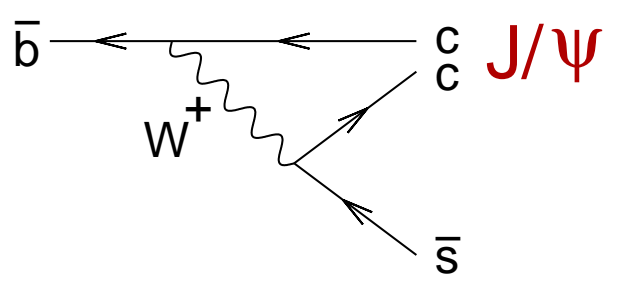

Figure 1.1: Bottom quark decay into a strange quark and a $\mathrm{J} / \psi$ meson. From left to right the magnitudes of coupling at the two $W^{+}$vertices are given by $V_{c b}^{*}$ and $V_{c s}$ respectively
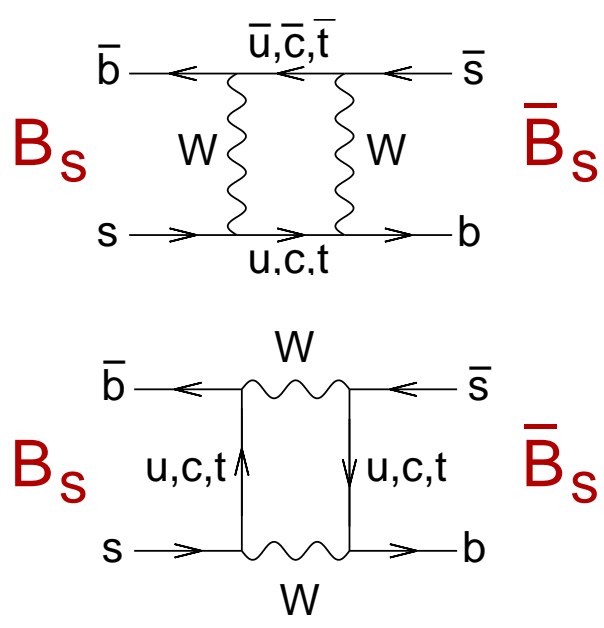

Figure 1.2: Box diagrams illustrating a $B_{s}^{0}$ mixing into a $\overline{B_{s}^{0}}$ meson

by the CKM matrix elements discussed in section 1.2.3. Charged mesons cannot mix: a statement which can be verified by attempting to draw the associated box diagram.

The process of mixing can be phenomenologically described by looking at the eigenstates of the two particles. In the mixing process the strong force (or flavour), eigenstates $\left|B^{0}\right\rangle$ and $\left|\overline{B^{0}}\right\rangle$ are no longer mass eigenstates and are coupled together by the mixing process. After creation of a $\left|B^{0}\right\rangle$ at time $t=0$, the meson state observed at time $t$ can be described by a superposition of the two flavour states:

$$
a(t)\left|B^{0}\right\rangle+b(t)\left|\bar{B}^{0}\right\rangle
$$

Since mixing takes place in the rest frame of the meson, the behaviour of the state can be described by the time-dependent Schrödinger equation:

$$
i \frac{d}{d t} \psi(t)=H \psi(t)
$$


In this system the two wavefunction components are $a(t)$ and $b(t)$.

$$
i \frac{d}{d t}\left(\begin{array}{l}
a \\
b
\end{array}\right)=\left(\boldsymbol{M}-\frac{i}{2} \boldsymbol{\Gamma}\right)\left(\begin{array}{l}
a \\
b
\end{array}\right)
$$

where $\boldsymbol{M}$ and $\boldsymbol{\Gamma}$ are 2x2 hermitian mass and decay matrices. CPT invariance guarantees $M_{11}=M_{22} \equiv M$ and $\Gamma_{11}=\Gamma_{22} \equiv \Gamma$, i.e. particle, anti-particle masses and lifetimes are identical. $M$ and $\Gamma$ correspond to the mass and decay width of the $B^{0}$ and $\overline{B^{0}}$ flavour states. Equation 1.3 has the following mass eigenstates:

$$
\begin{aligned}
& \left|B_{l}\right\rangle=p\left|B^{0}\right\rangle+q\left|\bar{B}^{0}\right\rangle \quad\left|B_{h}\right\rangle=p\left|B^{0}\right\rangle-q\left|\bar{B}^{0}\right\rangle \\
& i \frac{d}{d t}\left(\begin{array}{l}
a \\
b
\end{array}\right)=\left(\begin{array}{cc}
M-\frac{i}{2} \Gamma & M_{12}-\frac{i}{2} \Gamma_{12} \\
M_{12}^{*}-\frac{i}{2} \Gamma_{12}^{*} & M-\frac{i}{2} \Gamma
\end{array}\right)\left(\begin{array}{l}
a \\
b
\end{array}\right)
\end{aligned}
$$

It is convenient to define the mass and width differences:

$$
\Delta M=M_{h}-M_{l}, \quad \Delta \Gamma=\Gamma_{h}-\Gamma_{l}
$$

and the mixing parameter $x$ :

$$
x=\frac{\Delta M}{\bar{\Gamma}}
$$

where $\bar{\Gamma}$ is the mean width.

More discussion of neutral meson mixing and a more detailed approach can be found in [33], [15], [5] and [54].

\subsubsection{CP Violation in Mesons}

There are three ways to violate CP: (i) via mixing which results from the physical eigenstates being different from CP eigenstates; (ii) in the decay amplitudes, i.e. $B^{0} \rightarrow f$ and $\overline{B^{0}} \rightarrow \bar{f}$ decay amplitudes differ; and (iii) in the interference of mixing and decay amplitudes, which occurs in decays into final states that are common to $B^{0}$ and $\bar{B}^{0}$ mesons. In the bottom quark sector it is possible to make constraints on all types of CP-violation. The following text is a summary of possible CP-violation measurements in the bottom quark sector. 
CP Violation through mixing CP-violation in mixing occurs when the mass eigenstates, defined in equation 1.4, are different from the CP eigenstates. For this to be the case there must be a relative phase between the off-diagonal matrix elements $M_{12}$ and $\Gamma_{12}$, defined in equation 1.5.

A simple example of $\mathrm{CP}$ violation through mixing is the decay asymmetry of neutral mesons $B^{0}$ to leptonic states:

$$
a_{s l}(t)=\frac{\Gamma\left(B^{0}(t) \rightarrow l^{+} \nu X\right)-\Gamma\left(B^{0}(t) \rightarrow l^{-} \bar{\nu} X\right)}{\Gamma\left(B^{0}(t) \rightarrow l^{+} \nu X\right)+\Gamma\left(B^{0}(t) \rightarrow l^{-} \bar{\nu} X\right)}
$$

This asymmetry can be rewritten by using $q$ and $p$, defined in equation 1.4. $q$ and $p$ are related to the off-diagonal mass and width matrix elements by:

$$
\left|\frac{q}{p}\right|^{2}=\left|\frac{2 M_{12}^{*}-i \Gamma_{12}^{*}}{2 M_{12}-i \Gamma_{12}}\right|
$$

Substituting equation 1.8 into equation 1.7, a clearer parameterisation of the effect is given by:

$$
a_{s l}(t)=\frac{1-|q / p|^{4}}{1+|q / p|^{4}}
$$

From equation 1.9 it is clear that if $|q / p| \neq 1$ then CP violation is present.

CP Violation through decay If CP is conserved, then the decay rates for $B \rightarrow f$ and $\bar{B} \rightarrow \bar{f}$ must be equal. For example, the CP violation asymmetry for charged $B^{ \pm}$meson decays can be defined as:

$$
a_{f}=\frac{\Gamma\left(B^{-} \rightarrow f\right)-\Gamma\left(B^{+} \rightarrow \bar{f}\right)}{\Gamma\left(B^{-} \rightarrow f\right)+\Gamma\left(B^{+} \rightarrow \bar{f}\right)}
$$

where $f$ is the final CP state such that $|\bar{f}\rangle=C P|f\rangle$.

Parameterising the amplitudes of the final states as:

$$
A_{f}=\left\langle f \mid B^{+}\right\rangle, \text {and } \bar{A}_{\bar{f}}=\left\langle\bar{f} \mid B^{-}\right\rangle
$$

the $\mathrm{CP}$ asymmetry can be rewritten as:

$$
a_{f}=-\frac{1-\left|\bar{A}_{\bar{f}} / A_{f}\right|^{2}}{1+\left|\bar{A}_{\bar{f}} / A_{f}\right|^{2}}
$$

As stated in the previous section charged mesons cannot mix and therefore if $\left|A_{f}\right| \neq\left|\bar{A}_{\bar{f}}\right|$ $\mathrm{CP}$ violation must be present. This type of $\mathrm{CP}$ violation is commonly referred to as direct CP violation. 
CP Violation through the interference between decay and mixing Interference $\mathrm{CP}$ violation is present when the decays $B^{0} \rightarrow f_{C P}$ and $B^{0} \rightarrow \overline{B^{0}} \rightarrow f_{C P}$ interfere with each other, where $f_{C P}$ is a self-conjugate final state. This effect gives rise to the time dependent asymmetry:

$$
a_{f}(t)=\frac{\Gamma\left(\overline{B^{0}} \rightarrow f\right)-\Gamma\left(B^{0} \rightarrow f\right)}{\Gamma\left(\overline{B^{0}} \rightarrow f\right)+\Gamma\left(B^{0} \rightarrow f\right)}
$$

This time dependent CP asymmetry is present if there is $\mathrm{CP}$ violation due to mixing or $\mathrm{CP}$ violation in decay. The interference mixing is best described by introducing a new complex parameter $\lambda_{f}$ which is related to the other parameters used when describing CP violation by:

$$
\lambda_{f}=\frac{q}{p} \frac{\overline{A_{f}}}{A_{f}}
$$

Assuming the effects of these types of CP violation is small i.e. $|p / q| \approx 1$ and $\left|\overline{A_{f}} / A_{f}\right| \approx 1$ then $\left|\lambda_{f}\right|=1$ to a good approximation. Under this approximation it can be shown [5] that equation 1.11 becomes:

$$
a_{f}(t)=-\Im\left(\lambda_{f}\right) \sin \left(\Delta m_{B} t\right)
$$

This type of CP violation is already well established in $B^{0}$ decays in modes such as $B^{0} \rightarrow J / \psi K_{s}$.

\subsection{Motivation for this Thesis}

This section presents a summary of the reasons why studying $B_{s}^{0} \rightarrow J / \psi \eta$ is of importance for futher understanding flavour changing charged current interactions in the bottom quark sector.

\subsection{1 $B_{s}^{0}$ Width Difference}

Theoretical Summary The main motivation for the study of $B_{s}^{0} \rightarrow J / \psi \eta$ is to make a future measurement of the width difference $\Delta \Gamma_{B_{s}^{0}}$. The mass eigenstates $\left|B_{L}\right\rangle$ and $\left|B_{H}\right\rangle$ have different masses and different widths. The theoretical prediction of

$$
\Delta \Gamma_{B_{s}^{0}}=\Gamma_{L}-\Gamma_{H} \approx 2\left|\Gamma_{12}\right| \cos \phi_{s}
$$

is dependent on the calculation of $\left|\Gamma_{12}\right|$ and $\phi_{s}$, where $\phi_{s}$ is the relative phase between $M_{12}$ and $\Gamma_{12} . \phi_{s}$ is defined as:

$$
\phi_{s}=\arg \left(-\frac{M_{12}}{\Gamma_{12}}\right)
$$


The Standard Model predictions of $\phi_{s}$ constrains the value to be appoximately $0.2^{o}[5]$. The value of $\phi_{s}$ may be affected by new physics processes affecting cancellations in the calculation. A significantly larger angle would imply new physics outside the Standard Model. The calculation of the ratio $2\left|\Gamma_{12}\right| / \Gamma$ was also discussed in [5], where the value using current lattice calculations was given as:

$$
\frac{2\left|\Gamma_{12}\right|}{\Gamma}=0.12 \pm 0.06
$$

Measurement The width difference over the mean width can be expanded in terms of the light and heavy lifetimes:

$$
\frac{\Delta \Gamma}{\bar{\Gamma}}=\frac{2\left(\tau_{h}-\tau_{l}\right)}{\tau_{h}+\tau_{l}}
$$

where $\tau_{l}$ and $\tau_{h}$ are the light and heavy mean lifetimes. Assuming that modes can be found where the CP violation is small, $\mathrm{CP}$ odd and even eigenstates can be used to find these mean lifetimes. For a CP-even eigenstate $B_{s}^{0} \rightarrow J / \psi \eta$ or $B_{s}^{0} \rightarrow J / \psi \phi$ using CP-even part, can be used. Then the CP-even lifetime can be compared with a 50:50 mixed state such as $B_{s}^{0} \rightarrow D_{s}^{+} \pi^{-}$, to get a final value of $\Delta \Gamma / \bar{\Gamma}$.

\subsubsection{CP Asymmetry}

Time-dependent CP asymmetry may be observable in $B_{s}^{0}$ and $\overline{B_{s}^{0}}$ decays to the final common state $J / \psi \eta$, analogous to that observed in $B^{0}$ and $\overline{B^{0}}$ decays to $J / \psi K_{s}$. The CP assymetry, given by equation 1.12 , is expected to be small; it is proportional to $\Im\left(\lambda_{f}\right)$ which for this decay is $\sin \left(2 \beta_{s}\right)$. $\beta_{s}$ given previously in the discussion of the CKM matrix in section 1.2.3, is:

$$
\beta_{s} \equiv \arg \left(-\frac{V_{t s} V_{t b}^{*}}{V_{c s} V_{c b}^{*}}\right)=\mathcal{O}\left(\lambda^{2}\right)
$$

Future precise measurements of $B_{s}^{0} \rightarrow J / \psi \eta$ allow accurate determination of $\beta_{s}$. If this angle is larger than the order of $10^{-2}$ new CP-violating contributions to $B_{s}^{0} \overline{B_{s}^{0}}$ mixing must be present.

\subsection{Open Flavour Production}

From the previous sections it is clear why the avenue of bottom quark physics is of interest, but no discussion has been made of the environment in which the bottom quarks are 


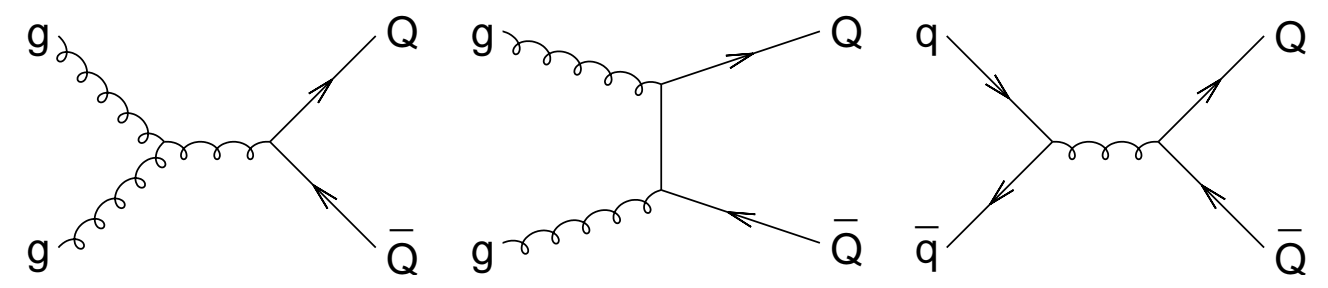

Figure 1.3: Tree level heavy quark flavour creation subprocesses.
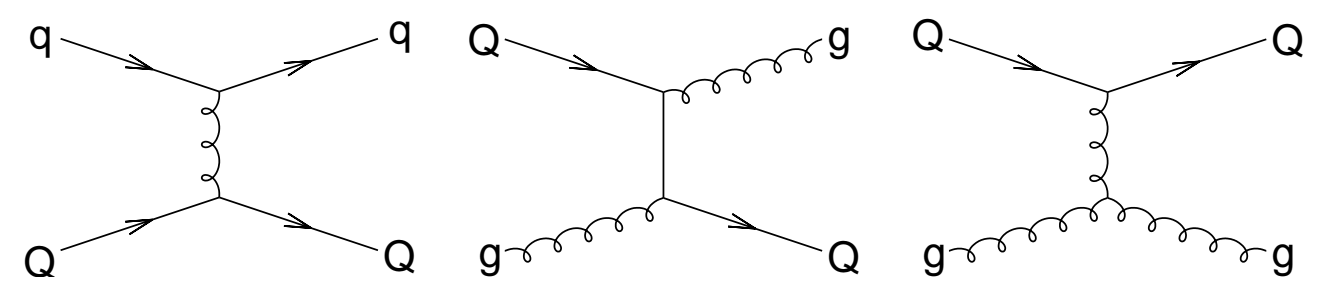

Figure 1.4: Tree level heavy quark flavour excitation subprocesses.

produced. Within a $p \bar{p}$ collider environment there are two lowest order types of $\mathrm{b}$ quark production: flavour creation and flavour excitation.

Flavour Creation Flavour Creation at lowest order describes the production of heavy quark pairs by the fusion of quark anti-quark.

$$
q \bar{q} \rightarrow Q \bar{Q}, \quad g \bar{g} \rightarrow Q \bar{Q}
$$

The lowest order Feynman diagrams for these QCD subprocesses are given in figure 1.3.

Flavour Excitation At the tree level there are also flavour excitation subprocesses which contribute to the total bottom quark production cross section. Within these processes an incident quark or gluon scatters a heavy quark from the sea into the final state.

$$
q Q \rightarrow q Q, \quad g Q \rightarrow g Q
$$

Some of the lowest order Feynman diagrams for these QCD subprocesses are given in figure 1.4 .

Parton Distribution Functions Protons and anti-protons are composite particles consiting of three quarks $(u u d)$ and anti-quarks $(\overline{u u} \bar{d})$ respectively. These quarks are 
bound together by gluons. Within the proton the quarks only carry a fraction of the momentum. The internal structure of the proton can be described via fits to scattering data by parton distribution functions (PDFs). These PDFs describe the probability for each parton within the proton to have a given momentum fraction. Measured PDFs will be discussed in section 4.2.2, as the one of the inputs into simulating B meson decays. 


\section{Chapter 2}

\section{Experimental Apparatus}

\section{$2.1 \quad$ Introduction}

Humanity's understanding of particle physics could not advance without the building of experimental facilities. Within a typical facility, high-energy beams are collided. Out of these collisions particles not seen since the beginning of the Universe are created. Detectors are positioned about the point of collision, to measure the properties of the particles produced.

The analysis for this thesis was carried out on data taken with the Collider Detector at Fermilab (CDF). CDF was constructed at the Fermi National Accelerator Laboratory (FNAL) in Illinois USA. When partially completed, the apparatus first detected $p \bar{p}$ collisions in October 1985. The experiment is positioned at a collision point for $p$ and $\bar{p}$ beams provided by the Tevatron, a superconducting accelerator. Over the past three decades the experimental apparatus has been subject to modifications improving its performance. The experimental apparatus described in the following text is discussed as it was during the Run I time period (1992 to 1996).

\subsection{The Tevatron}

At the beginning of the acceleration chain, hydrogen atoms are stripped of their electrons by an electric field. The same electric field causes the resultant protons to cover the surface of a caesium electrode. Further incoming protons then knock off previously deposited protons from the caesium surface. Protons that are knocked off pick up two electrons, 
available due to caesium's low work function. These now $H^{-}$ions then drift away from the caesium electrode and are extracted for acceleration.

The first acceleration stage is a Cockcroft-Walton accelerator. A Cockcroft-Walton is a voltage multiplication ladder network. The Cockcroft-Walton at FNAL produces an accelerating potential difference of $750 \mathrm{kV}$. This potential is limited by discharge between the ladder elements. The Cockcroft-Walton is used to accelerate a steady stream of $\mathrm{H}^{-}$ ions collected from the Caesium electrode to an energy of $750 \mathrm{KeV}$. These $H^{-}$ions are then injected into a pulsed linac, made up of two sections: a drift tube linac and a side coupled linac. The drift tube linac is made up of five tanks, each driven by Tetrodes at $201 \mathrm{MHz}$. The Tetrodes produce electromagnetic waves causing the tanks to resonate a longitudinal mode. The tanks contain post couplers to reduce transverse modes. At the end of the drift linac the $H^{-}$ions have an energy of $116 \mathrm{MeV}$. The beam is then extracted and compressed to match a $805 \mathrm{MHz}$ RF structure. With the new RF structure the $H^{-}$ ions are then fed into the side coupled linac. The side coupled linac was constructed from seven side coupled cavities driven by klystrons. At the end of the linac the beam reaches $400 \mathrm{MeV}$ and is ready to be injected into the Booster.

The Booster is a synchrotron. It was constructed from a set of dipole magnets for bending the beam around the circumference, quadropoles for focusing the beam, and RF cavities for accelerating the beam. A single pulse from the linac fills more than one rotation of the Booster. The nature of the $H^{-}$ions was then used to reduce the chances of kicking out protons already within the booster at the injection point. As the $H^{-}$beam joins the proton beam contained within the booster, the opposite charge causes the two beams to merge as they pass through the first dipole. The two beams then pass through a carbon stripping foil. While thin enough not to affect the proton beam, the foil is affective at stripping away the electrons leaving protons, hydrogen atoms and a residuum of $\mathrm{H}^{-}$ions. At the next dipole magnet any $H^{-}$ions that are left are ejected. By the end of Booster acceleration, the final energy at the extraction point is $8 \mathrm{GeV}$. Protons from the Booster were injected into the Main Ring, before passing into the Tevatron itself.

The Main Ring was a Synchrotron which, when operated with the Tevatron and CDF, ran at a peak energy of $150 \mathrm{GeV}$. Protons extracted at $120 \mathrm{GeV}$ from the main ring were directed onto a nickel target. Anti-protons were then collected with a lithium lens and transfered to a debuncher ring at operating at $8 \mathrm{GeV}$. The lithium lens is used to catch as 
many of the anti-protons as possible. It is constructed from a hoop of lithium metal and it driven by a pulsed electric current which induces an solinoidal field. The lithium lens is used in preference to two dipoles to maximise anti-proton acceptance.

Protons collected by the lithium lens are fed into the debuncher which uses RF cavities to rotate the anti-proton phase space, swapping a large energy spread and good time distribution for a wide time distribution and a narrow energy spread. From the debuncher ring the anti-protons were transfered into an accumulator ring also operated at $8 \mathrm{GeV}$. In the accumulator ring stochastic cooling and stacking takes place. After several hours there were enough anti-protons for re-injection into the main ring. Protons and antiprotons were then simultaneously ramped up to $150 \mathrm{GeV}$ for injection into the Tevatron. The steps of acceleration were illustrated in the schematic figure 2.1.

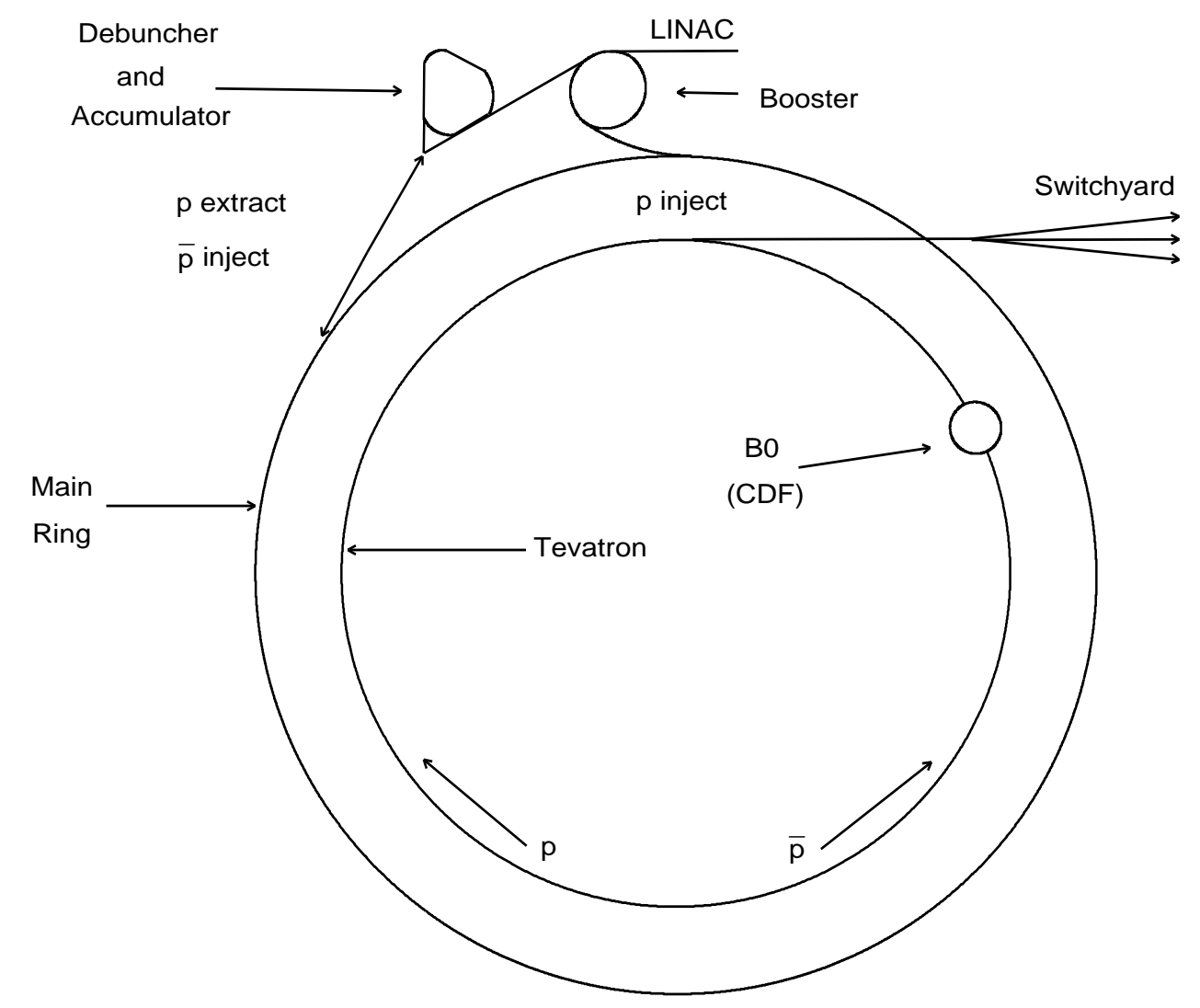

Figure 2.1: The Tevatron with pre-accelerators

The Tevatron, described in [26], was originally constructed as an energy saver: 
formed from superconducting magnets to reduce main ring running costs. At the time of construction it was the first large machine to use superconducting magnets. Since this was a research and development machine in every sense, it was decided that the superconducting structure would be housed inside the existing main ring tunnel. The Tevatron, once completed, received its first $150 \mathrm{GeV}$ protons in 1983. During the Run I period of operation it accelerated proton and anti-proton beams up to an energy of 0.9 $\mathrm{TeV}$ per beam. Without these beams no particle physics studies at CDF would have been possible.

\subsection{The Collider Detector at Fermilab}

\subsubsection{Introduction}

The design of the CDF detector, illustrated in figures 2.2 and 2.3, is similar to that of many high-energy colliding-beam detectors. It was aligned so that the interaction point, between proton and anti-proton beams was directly at the centre. As charged particles produced from the interaction of the two particle beams move away from the interaction point they twist outwards in the detector's magnetic field. These particle trajectories form helical paths, measured by layers of tracking chambers. Data from the tracking chambers were fitted to extract helix parameters for each track. From the track fits, vertex and momentum measurements were made. Outside the tracking chambers and the solenoid used to produce the magnetic field there are two layers of calorimetry. The first layer, made up of lead and scintillator, absorbs all the energy from the electrons and photons, and thus is referred to as the electromagnetic calorimeter. Hadrons continue to shower though into a second layer of calorimetry. The second layer, normally referred to as the hadronic calorimeter, was fabricated from steel and scintillator. Most of the hadronic energy from an interaction is deposited in the hadronic calorimeter. Occasionally some of the final parts of hadronic showers escape and pass through the inner most muon chambers. This is commonly referred to as hadronic punchthrough. The only particles that consistently escape the hadronic calorimeter are muons. These muons provide an essential means by which interesting physics processes can be selected from underlying soft interactions. In order to track the flight of muons outside the hadronic calorimeter, surrounding the central hadronic calorimeter barrel there are a layer of drift chambers. Around the first layer of 
central muon chambers there is the return yoke for the solenoid magnetic field, which also serves as further absorber for any hadronic punch-through. The steel return yoke was fabricated in a box shape, about which there were four flat layers of muon drift cells. To increase further the central muon coverage, muon detectors were added in two large arches, at an inclination of 45 degrees, on either side of the magnet return yoke.

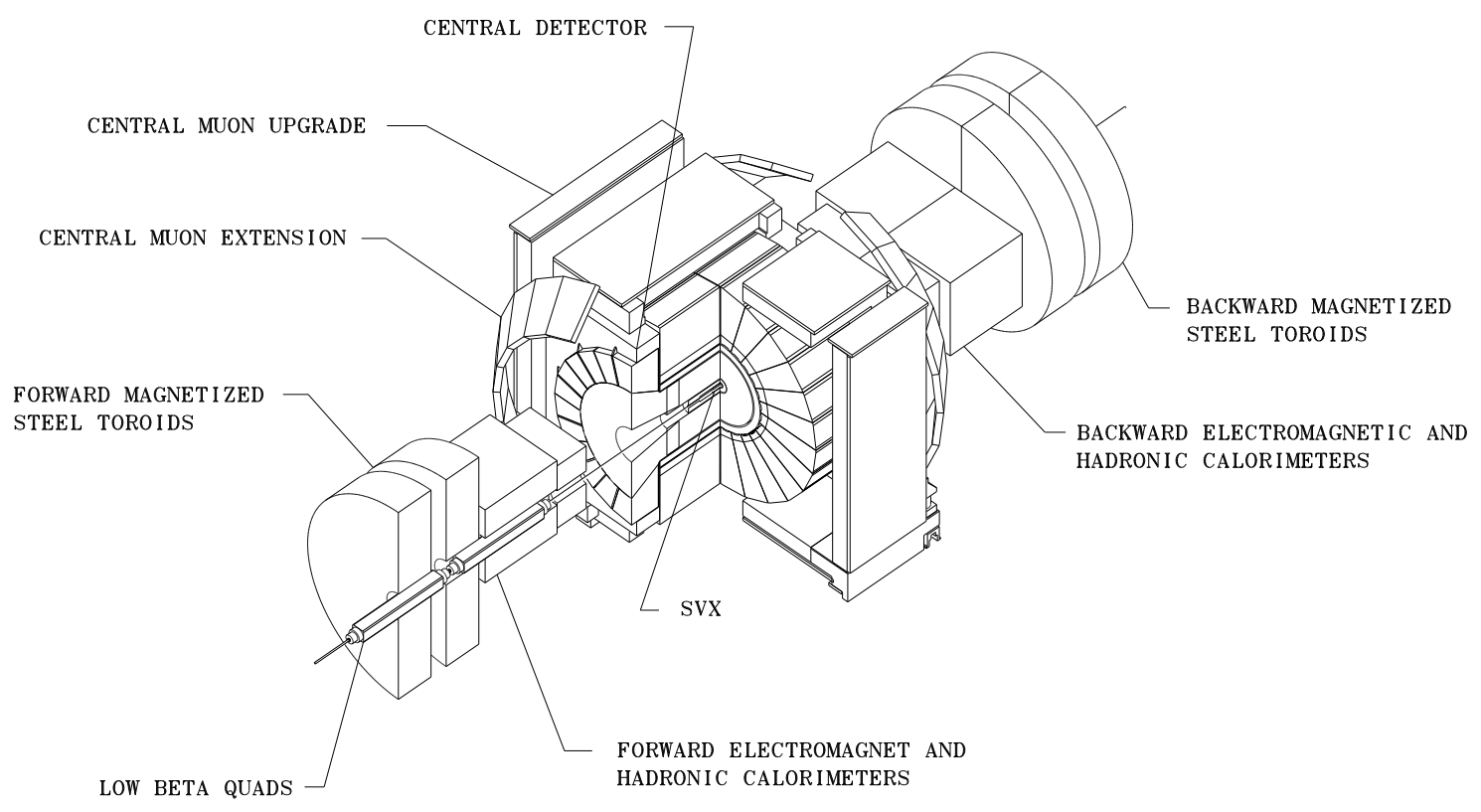

Figure 2.2: The CDF detector during the Run I period of 1992 to 1996

\subsubsection{Tracking}

At the centre of CDF there were tracking systems to measure the momentum and position of charged particles produced in a $p \bar{p}$ collision. All of the tracking systems were mounted inside a $3 \mathrm{~m}$ diameter, $5 \mathrm{~m}$ long super conducting solenoid. The solenoid produces a magnetic field of 1.4 Tesla, with the field lines parallel with the beam axis. Inside the solenoid there were four different tracking detectors: a silicon vertex detector (SVX), a VerTeX time projection chamber (VTX), and a central tracking drift chamber (CTC). All of these tracking systems are drawn in profile inside the CDF detector in figure 2.3.

\subsubsection{Silicon Vertex Detector}

Construction Immediately around the beam pipe the silicon vertex detector (SVX) was attached such that the innermost layer was at a radius of $29 \mathrm{~mm}$ from the centre of the 


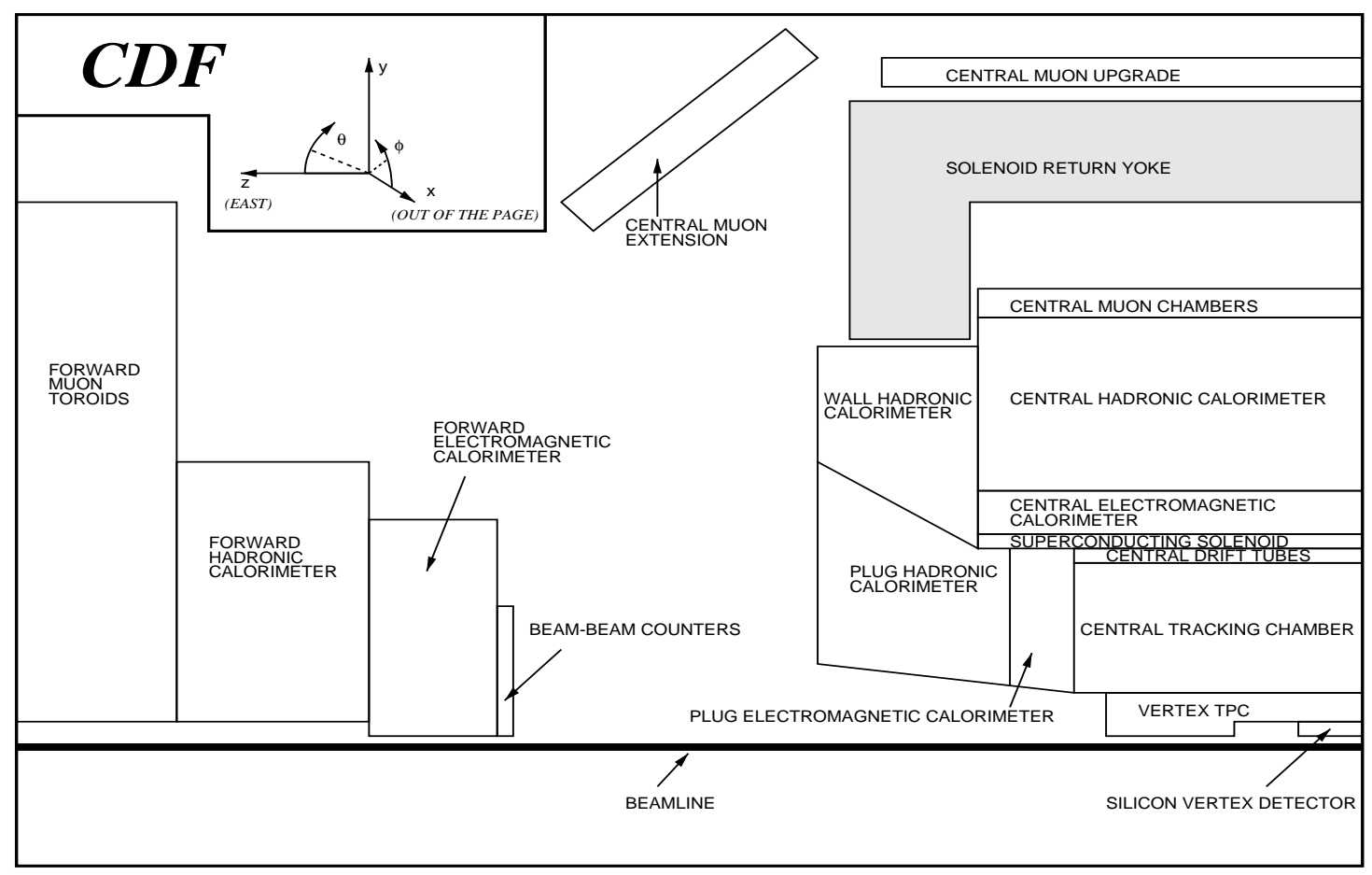

Figure 2.3: The CDF detector during the Run I period of 1992 to 1996. The illustration shows one quarter of the detector. The complete detector was symmetric about $z=0$ and $y=0$.

beam pipe. This device, described in [4] and [7], was constructed to tag long-lived heavyflavour particles produced in $p \bar{p}$ collisions. Such long-lived particles typically travel a few hundred microns before decaying. To measure the displacement of the secondary vertex with respect to the primary vertex, good spatial resolution is essential. Silicon micro strips provide adequate positional measurements to make this possible. The length of the SVX was made up of two barrels, each holding 4 radial layers of silicon strips parallel to the beam axis. Individual layers within a barrel were constructed from 12 ladders. Each ladder was made from three single sided silicon micro strip detectors aligned such that the strips ran parallel to the beam axis. The inner three layers of the SVX were constructed from $60 \mu \mathrm{m}$ pitch micro strips and the the outer layer was constructed from $110 \mu \mathrm{m}$ pitch micro strips. At the outside end of each ladder a small circuit board called an ear was attached. The ear contained the readout chips and supporting electronics and was thermally isolated to prevent heating of the sensors by the electronics. Signals processed by the readout chips 
were relayed together with incoming bus signals on flexible kapton cables. For mechanical support and alignment a second Ear without electronics was attached to the other end of each ladder. Figure 2.4 shows a typical ladder complete with Ears and readout chips.

Once the silicon ladders had been constructed they were attached to a low mass space frame. Multiple scattering is a source of tracking uncertainty and the amount of material used for these support structures was kept to a minimum. Each ladder was mounted on a foam and carbon fibre support which in turn was attached to an aluminium bulkhead machined from one piece of aluminium. Then, when all of the wedges had been assembled and attached to the two barrels the complete device was covered by an electrical conductive skin, to reduce interference from electromagnetic noise sources, and to add extra structural integrity. After assembly the total material included equated to an average of $3 \%$ of a radiation length. One partially assembled barrel is shown in figure 2.5.

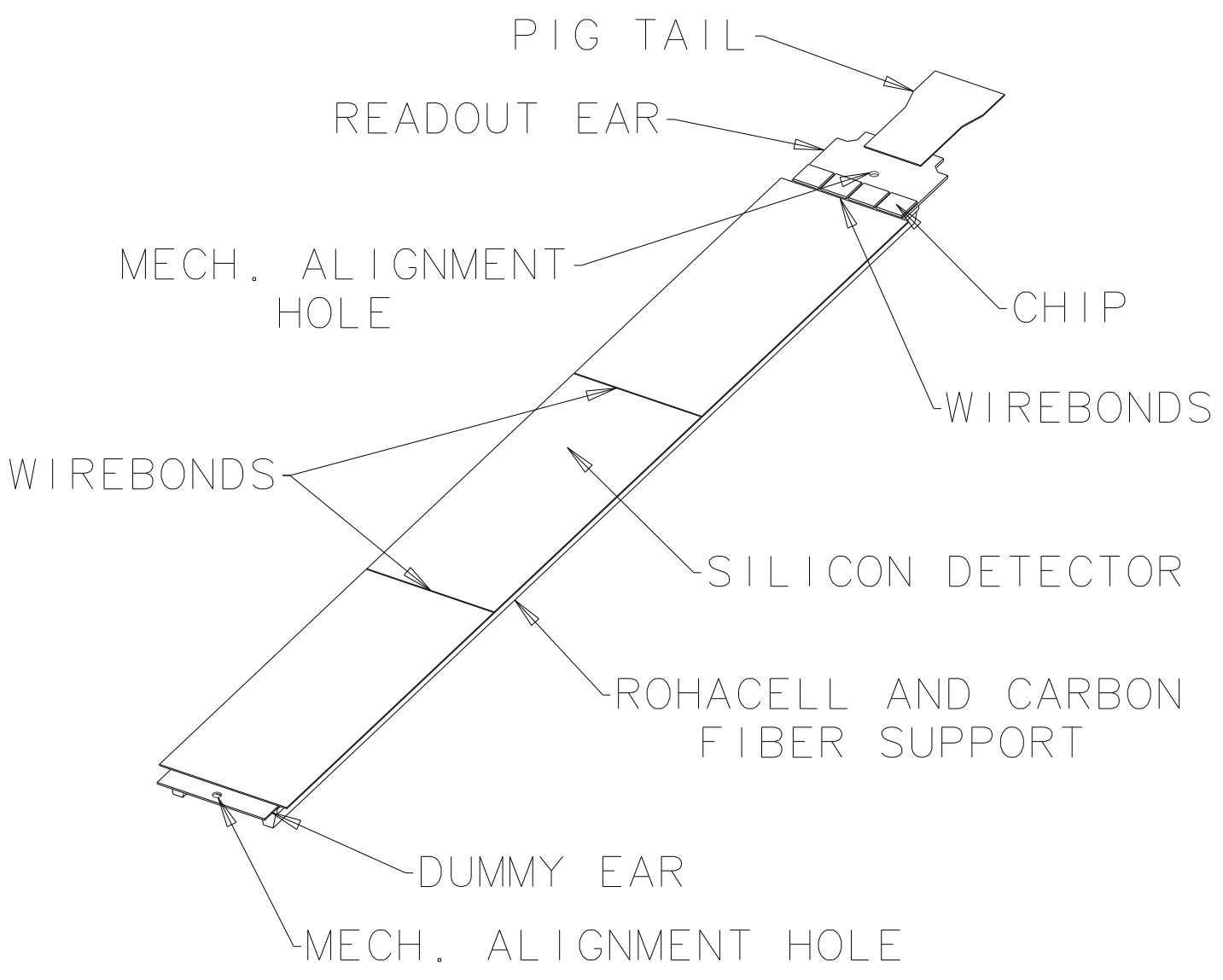

Figure 2.4: A typical Silicon Vertex detector ladder. 


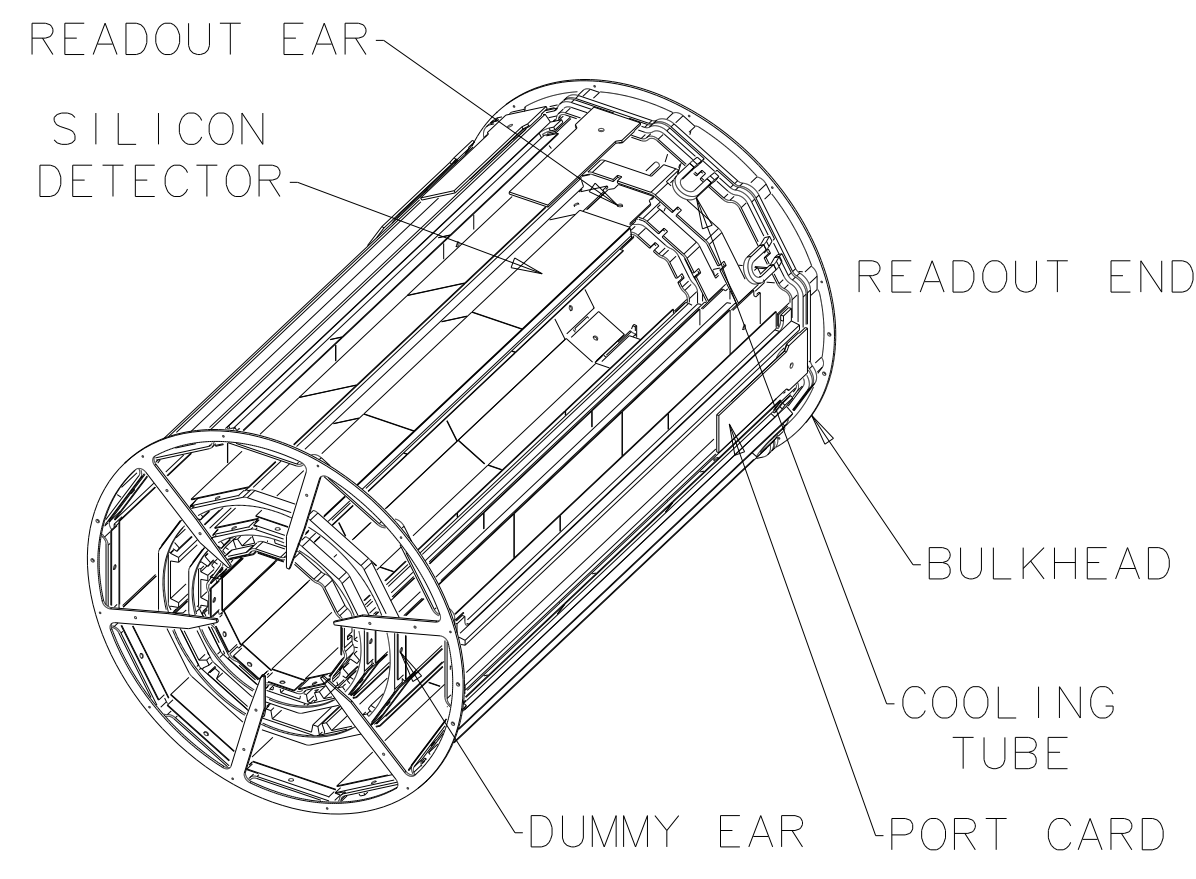

Figure 2.5: A schematic diagram of one of two Silicon Vertex detector barrels showing all four layers of silicon together with aluminium bulk-heads. The two barrels when coupled together formed a $60 \mathrm{~cm}$ active region.

Properties During the period of running from 1992 to 1996 the SVX had to be replaced once due to radiation damage. The original SVX was produced from single sided, DCcoupled microstrip detectors, $8.5 \mathrm{~cm}$ in length. The replacement detector called SVX(') was geometrically the same except for a few small adjustments. The inner layer was modified such that the ladders were tilted by 1 degree to overlap the silicon, and was moved closer to the beam pipe by $1.5 \mathrm{~mm}$. Taking advantage of technological advances, single-sided AC-coupled silicon micro strip sensors were used. To read out the silicon sensors a new readout chip was fabricated, with a more radiation tolerant process. AC coupling implied that that only one integration step was required instead of two, instantly reducing the noise by $\sqrt{2}$ with respect to SVX. An additional benefit of AC coupling was that the leakage current was no longer visible at the pre-amp input and so saturation could be 


\begin{tabular}{|l|c|c|}
\hline \hline Design Parameter & SVX & SVX(') \\
\hline Channels & 46080 & 46080 \\
Coverage Along Z & $51.1 \mathrm{~cm}$ & $51.1 \mathrm{~cm}$ \\
Noise & 2200 electrons & 1300 electrons \\
Gain & $15 \mathrm{mV} / \mathrm{fC}$ & $21 \mathrm{mV} / \mathrm{fC}$ \\
Radiation Limit & $15-20 \mathrm{KRad}$ & $>1 \mathrm{MRad}$ \\
\hline \hline
\end{tabular}

Table 2.1: Comparison of SVX and SVX(')

avoided. Both detectors are summarised in table 2.1 .

\subsubsection{Vertex Time Projection Chamber}

The vertex time projection chamber (VTX), most completely described in [42], was designed to fit inside the central tracking chamber and allow room for a silicon vertex detector. The VTX was $2.8 \mathrm{~m}$ in length and had an outer radius of $25 \mathrm{~cm}$. The time projection chamber was built to measure the position of vertices along the beam axis.

The chamber was divided up into twenty eight $10 \mathrm{~cm}$ modules along the beam line. Each module was subdivided into eight octants in $\phi$. Through the middle of each octant, from one radial wall to the other, sense wires were strung, splitting the module into two drift regions. The disk-shaped modules were assembled to be out of phase with respect to their neighbour by 15 degrees of azimuth. This offset provided limited azimuthal information for tracks passing through two modules. On either side of the centre of the detector the first 9 modules were fabricated with 16 sense wires and an inner radus of $20 \mathrm{~cm}$. The next ten modules, either side of the silicon detector, had an inner radii of $10 \mathrm{~cm}$. A graphic representation of the VTX detector is given in figure 2.6.

Once complete and installed the chamber was filled with argon/ethane in the ratio $50 / 50$. When operated the resultant vertex resolution along the beam axis, combined with the central tracking chamber was $2 \mathrm{~mm}$.

\subsubsection{Central Tracking Chamber}

Travelling radially outwards, the Central Tracking Chamber (CTC), encompassed the vertex time-projection chamber. The CTC, described in [11], was a gas drift chamber 

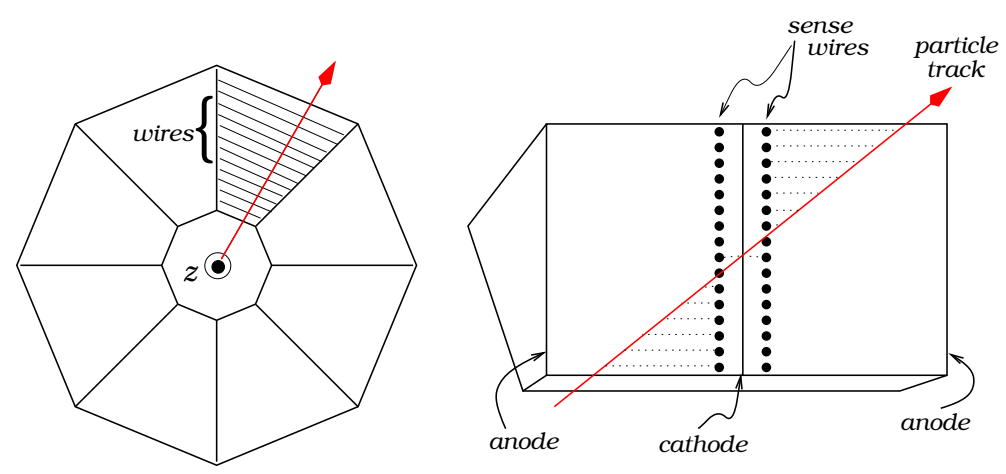

Figure 2.6: The Vertex Time Projection Chamber, showing sense wires and octants [38].

designed to measure both charge particle track positions both with the transverse plane and along the beam line. The chamber was made up of 9 assemblies of wires called superlayers. 5 of these superlayers were strung such that the wires ran axially, that is along the beam axis. The other 4 superlayers were strung in alternate 3 -degree stereo with respect to the axial wires. Axial and stereo superlayers were arranged alternately, radially starting and ending with axial superlayers. Each superlayer was subdivided into drift cells. The drift cells were designed such that the maximum drift length was $40 \mathrm{~mm}$ which was approximately equivalent to a drift time of 800ns. Including the end plate, the chamber was $2760.0 \mathrm{~mm}$ in diameter and $3201.3 \mathrm{~mm}$ long. In terms of tracking coverage, the chamber covers $\phi=0 \rightarrow 2 \pi$ and $|\eta|=0 \rightarrow 1.16$ for an exit radius of $110 \mathrm{~cm}$. The assembled CTC end plate is illustrated in figure 2.7.

In the CTC electric and magnetic fields were perpendicular to each other. In this environment electrons produced when a charged particle ionises the gas mixture, drift at an angle of approximately the Lorentz angle, defined in equation 2.1.

$$
\beta=\tan ^{-1}\left(\frac{v(E, B=0) B}{k E}\right),
$$

where $v(E, B=0)$ is the drift velocity with no magnetic field, $B$ is the magnetic field measured in Tesla, $E$ is the electric field measured in $V m^{-1}$, and $k$ depends on the gas mixture.

The Lorentz angle was accommodated for and the drift relation linearised by setting the wires in each superlayer at an angle. The wires were set such that the electric field was at 45 degrees to the radial vector. The resulting drift direction was approxi- 


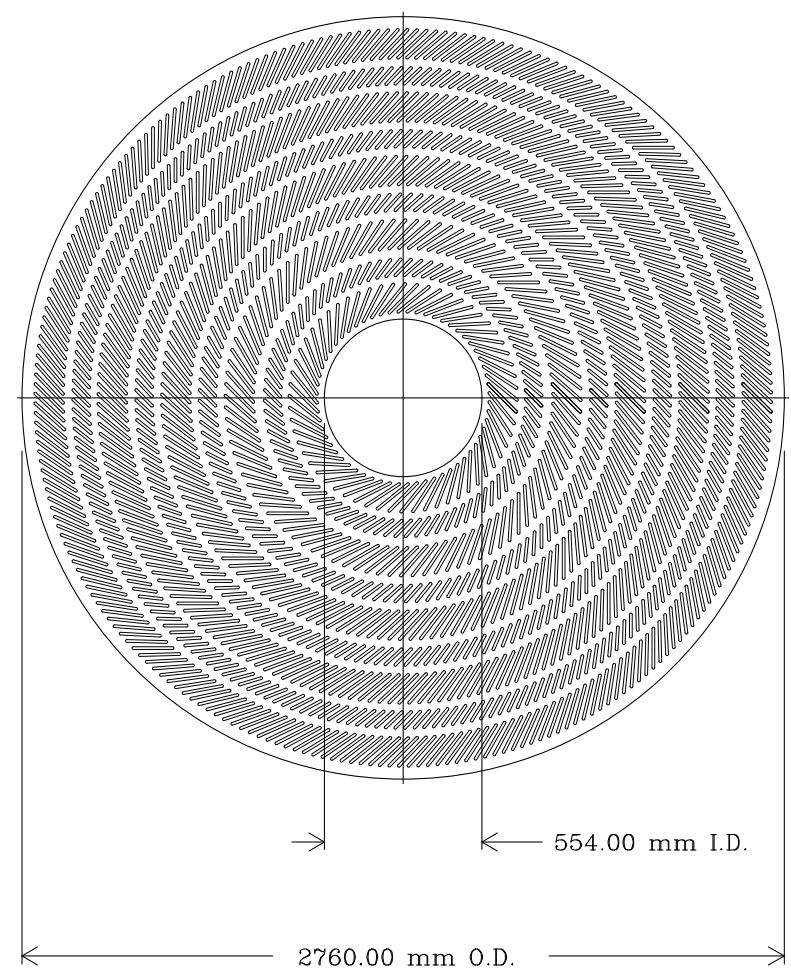

Figure 2.7: The Central Tracking Chamber end plate. Regions superlayer wires were connected are shown. The Axial superlayers are composed of less wires

mately azimuthal. The main advantage of this large incline was that tracks with high momentum i.e. travelling approximately radially outwards, pass close to a sense wire in each superlayer. This is especially useful when there are many tracks which have to be resolved. When fully assembled the detector was filled with a gas mixture of $49.6 \% / 49.6 \% / 0.8 \%$ argon/ethane/ethanol. The transverse momentum resolution was found to be $\delta p_{t} / p_{t}=0.001 \times p_{t}$, and the resolution along the beam axis was found to be approximately $4 \mathrm{~mm}$.

\subsubsection{Calorimetry}

The CDF calorimeter systems were divided up into three sections: the central, plug and forward. Neither the plug nor forward calorimeter systems were used during the analysis contained in this thesis and further information can be found in [16] and [30]. 


\subsubsection{Central Electromagnetic Calorimeter}

The central calorimeter contains both hadronic and electromagnetic segments. As particles produced by a $p \bar{p}$ interaction travel radially outwards, in a pseudo-rapidity range $|\eta|<1.1$, they enter the Central Electromagnetic Calorimeter (CEM). The CEM, described in [9], is a sampling calorimeter made of $1 / 8$ inch lead plates and $5 \mathrm{~mm}$ SCSN-38 polystyrene sheets. The CEM was constructed from two halves split at the centre of the interaction point. Each half was built from 24 wedges around the azimuthal angle $\phi=0$ to $2 \pi$. With the exception of one module, discussed later, all were internally subdivided into 10 pointing tower sections of dimensions $\Delta \phi=\pi / 12$, and $\Delta \eta=0.1$.

Inside each module, between the 8 th lead layer and 9 th scintillator layer, a proportional chamber (CES) was positioned to measure the position and energy of the electromagnetic shower centroid. Each tower was constructed so that the number of radiation lengths from the solenoid to the CES was approximately equal. That is a particle produced at the centre of the interaction region travelling outwards through the CEM would see roughly the same number of radiation lengths exclusive of which tower it entered. This design requirement was implemented by varying the amount of lead in each tower. At tower boundaries further from $|\eta|=0$ some of the lead sheets were truncated and the remaining space was filled with acrylic sheets of the same dimension. The configuration of each tower is tabulated in table 2.2 .

The scintillation light from each tower was collected by acrylic waveshifters. These waveshifters were attached on both sides of each tower. The material chosen for light collection was $3 \mathrm{~mm}$ UVA acrylic doped with $30 \mathrm{ppm}$ Y7. When assembled the waveshifters collected blue scintillation light and shifted it to green light. Each wave shifter was glued to a UVA acrylic light guide rod, carrying the green light to Hamamatsu R580 photo multipliers. A typical module complete with wave shifters and photo multipliers was illustrated in figure 2.8. The complete detector was found to have an average electromagnetic energy resolution $\sigma(E) / E$ of $13.5 \% \sqrt{E \sin (\theta)}[9]$ where $E$ has the units of $\mathrm{GeV}$.

Chimney Tower As previously mentioned 47 of $48 \mathrm{CEM}$ modules were the same. The odd module was constructed to allow a two-phase liquid-helium cooling line to run down to the solenoid magnet. This CEM module was notched making it 12 inches shorter than 


\begin{tabular}{|c|c|c|c|c|}
\hline \hline Tower & Angle & To CES $\left(X_{0}\right)$ & Total Stack $\left(X_{0}\right)$ & Coil $\left(X_{0}\right)$ \\
\hline 0 & 86.3 & 4.9 & 17.9 & 0.86 \\
1 & 79.15 & 5.0 & 18.2 & 0.88 \\
2 & 72.2 & 5.1 & 18.2 & 0.90 \\
3 & 65.65 & 4.8 & 17.8 & 0.95 \\
4 & 59.75 & 5.0 & 18.0 & 1.00 \\
5 & 54.5 & 4.6 & 17.7 & 1.06 \\
6 & 49.85 & 4.9 & 18.1 & 1.13 \\
7 & 45.9 & 4.5 & 17.7 & 1.20 \\
8 & 42.2 & 4.8 & 18.0 & 1.28 \\
9 & 39.9 & 5.0 & 10.0 & 1.34 \\
\hline \hline
\end{tabular}

Table 2.2: Number of radiation lengths for central electromagnetic calorimeter stack up and solenoid coil.

a regular tower. The notched module contained seven standard towers and one combined tower, referred to as the chimney tower. Since the chimney tower was notched to allow for the cryogenic feed, there was room for only one wave shifter to be attached.

Calibration The central electromagnetic calorimeter had three calibration devices [34]: a $C s^{137}$ source, a xenon flash lamp and a LED array. Each module had its own $3 \mathrm{mCi}$ $C s^{137}$ source, which could be driven through a module at a constant speed. The $C s^{137} \mathrm{X}-$ ray source deposited energy in the scintillator sheets, which was used to update test beam calibration maps of the modules. Xe flash lamps were connected to the beginning of each wave shifter by quartz fibres. These were used to measure the degradation of the wave shifters over time. In the third calibration system, a bank of green LEDs was connected to a small piece of wave shifter material positioned just in front of each photomultiplier. Both the Xe and LED flasher calibration systems were monitored by PIN diodes.

\subsubsection{Proportional Strip Chambers}

Within each electromagnetic calorimeter module between the 8th lead layer and 9th scintillator layer a Proportional Strip Chamber (CES) was positioned at approximately 6 radiation lengths from the centre of the detector. This position corresponds to the 


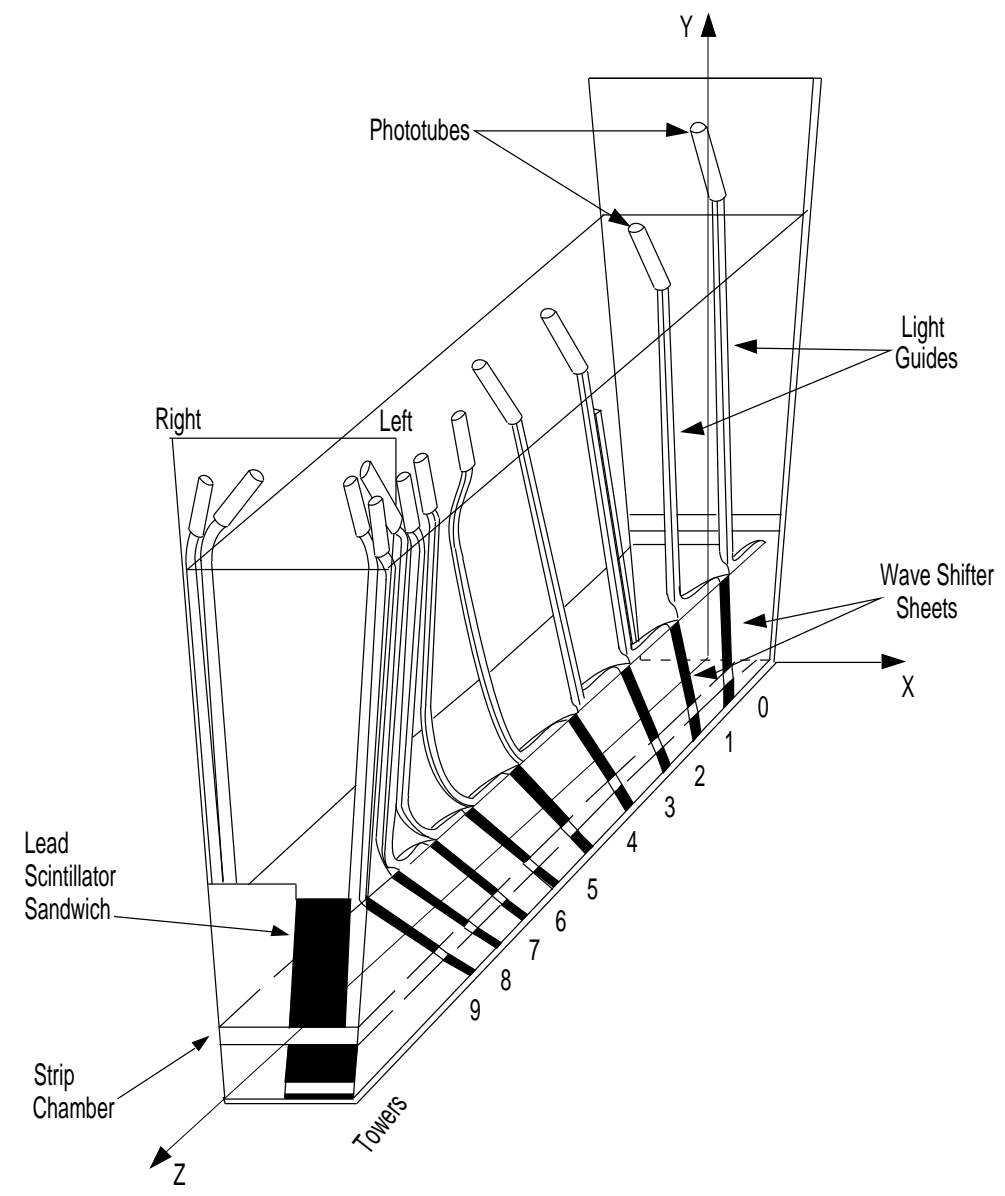

Figure 2.8: A typical Central Electromagnetic Calorimeter module.

electromagnetic shower maximum for a $10 \mathrm{GeV}$ electron. The CES, described in detail within [53], was designed to record the position and development of an electromagnetic shower. CEM towers are large, and therefore could contain more than one electromagnetic shower. The CES was used to distinguish between multiple showers within one tower. It was also used to separate hadronic and electromagnetic showers by comparing the pulse height to the tower energy.

Except for the CES chamber inside the chimney tower each chamber was $233.4 \mathrm{~cm}$ long, $45.0 \mathrm{~cm}$ wide, and was located $185 \mathrm{~cm}$ from the beam line. Inside the chamber there were 64 anode wire channels and 128 cathode strip channels. Apart from the wires located immediately adjacent to the side walls of the strip chamber, each wire channel was made up from two individual wires forming a $14.53 \mathrm{~mm}$ cell. These wires ran parallel to the beam axis and were divided at the interface between the 4th and 5th tower (counting 
from zero). Within the first section starting close to $\eta=0$, there were 69 cathode strips, evenly spaced and of width $16.67 \mathrm{~mm}$. In the second half of the module there were 59 evenly spaced strips of width $20.07 \mathrm{~mm}$. Within the CDF detector as a whole there were 47 CES modules all the same length. In the CEM module containing the chimney tower the CES did not extend into the chimney tower.

When assembled all chambers were filled with 95\%/5\% Argon/Carbon Dioxide. In this environment wire and strip gains were set such that they were correlated to the degree of 6-15\% [9], enabling ambiguities to be removed. Spatial and energy resolution was found to be non-linear due to the single-sample nature of the chamber. The electromagnetic energy resolution was 20 to $30 \%$, whereas the positional was $2-10 \mathrm{~mm}$ depending on the energy [9].

\subsubsection{Central and End Wall Hadronic Calorimeters}

The Central Hadronic Calorimeter (CHA) and the End Wall Calorimeter (WHA), both described in [14], covered a range of $|\eta|<1.3$ and azimuthal of $2 \pi$. Both calorimeters were segmented into $\pi / 12$ azimuthal sections, matching the electromagnetic calorimeter.

The construction material chosen for these sampling calorimeters was steel and scintillator. Each projective module was constructed to fill $\Delta \eta=0.1$, and $\Delta \phi=\pi / 12$. A CHA module had 9 towers. Moving out with respect to $\eta$, the last three towers were shared between the CHA and the WHA. The WHA was subdivided into six towers.

Unlike the central electromagnetic calorimeter, the scintillator was not a continuous sheet, and each layer was made up of individual tower sections. Scintillating layers where fabricated from $1 \mathrm{~cm}$ thick PPMA plastic doped with $8 \%$ naphthalene, $1 \%$ butyl-PBD and $0.01 \% \mathrm{POPOP}^{1}$. Around the edges of each scintillating tile tower section, $0.5 \times 1.0 \mathrm{~cm}$ UVA PMMA strips where attached. These plastic light-guide strips were doped with laser dye number 481 , to shift the scintillation light to $490 \mathrm{~nm}$. Strips of wave shifter were in turn connected to light guides running up both sides of each tower to two Thorn-EMI photo multipliers.

The absorbing steel layers were chosen to be $2.5 \mathrm{~cm}$ thick for the central and $5.0 \mathrm{~cm}$ thick for the end-wall calorimeter. A thicker plate was chosen for the end wall because on average more energy is deposited there. The final energy resolution for the central hadronic

\footnotetext{
${ }^{1}$ 1,4-Di-(2-(5-Phenyloxazolyl))-Benzene
} 
calorimeter was measured with pions to be $\sigma(E) / E=4 /(E \sin (\theta))+33 \% \sqrt{E \sin (\theta)}$.

Calibration Both central hadronic calorimeters were equipped with three calibration systems. Each module had a $3 \mathrm{mCi} C s^{137} \mathrm{X}$-ray source which could be driven through at a constant speed to provide updates to test beam calibration information.

Isolated calibration of photo-multiplier effects was done using a UV-emitting nitrogen laser to produce scintillation light. The scintillation light was initially produced in a separate piece of scintillator called a light distribution box. Light from the distribution box was then wavelength shifted and injected into the front of each photo-multiplier. In total six light distribution boxes were attached to the calorimeter.

\subsubsection{Muon Chambers}

The CDF detector has many sets of muon chambers to detect muons escaping from the hadronic calorimeter. Around the outside of the hadronic calorimeter three central muon chamber systems were attached: the Central Muon Detector (CMU), the Central Muon Upgrade (CMP), and the Central Muon Extension (CMX). The complete coverage map is shown in figure 2.9 .

\subsubsection{Central Muon Detector}

The design of the central muon detector (CMU) was published in [6]. The CMU was constructed from four layers of rectangular drift cells. Each drift cell's active region measured $63.5 \mathrm{~mm}$ wide, $26.8 \mathrm{~mm}$ deep and $2261 \mathrm{~mm}$ long. In the centre of the active region was a single $50 \mu \mathrm{m}$ sense wire. Muons were identified by their ability to reach the chambers after crossing 4.9 absorption lengths of calorimeter material.

The CMU detector was designed to measure position along the beam axis by using charge division, and around the azimuth by measuring drift times. Charge division measurements were performed by attaching an ADC to both ends of each sense wire. The resistance of the sense wire implied a different amount of charge was seen at each end, depending on the longitudinal position of the shower induced by the incident muon. This is the principle of charge division.

To remove the ambiguity from the stacked muon chambers every other chamber's sense wire was offset by $2 \mathrm{~mm}$. Operating the chambers with 50/50 Argon/Ethane in limited 


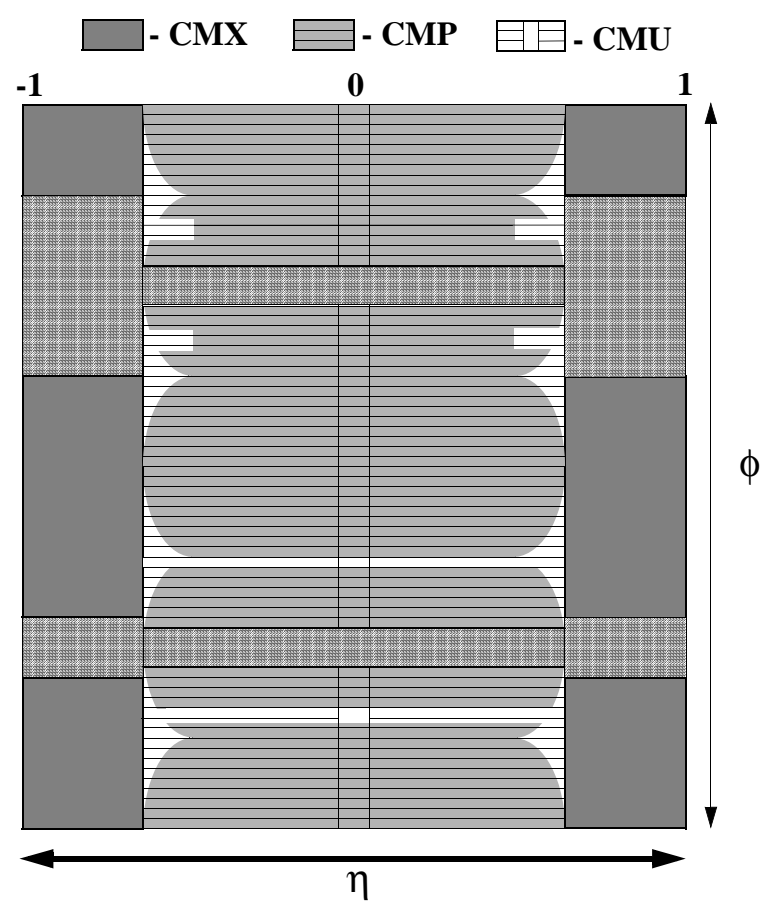

Figure 2.9: A plot summarising central muon coverage for the run period 1992-96. Plain gray patches are un-instrumented regions.

streamer mode produced a positional resolution of: $250 \mu \mathrm{m}$ along the drift direction and $1.2 \mathrm{~mm}$ from charge division.

The muon detector was divided up into 12.6 degree azimuthal sections which were assembled on top of the Central Hadronic Calorimeter Towers. Since CHA towers were divided into 25 degree azimuthal sections this left a 2.6 degree un-instrumented gap. The 12.6 degree azimuthal coverage was spanned by three CMU modules bolted together. Each module was constructed from an array of $4 \times 4$ rectangular drift chambers stretching down the $2261 \mathrm{~mm}$ length of the CHA module. The end of a single module complete with a muon track is illustrated in figure 2.10 .

\subsubsection{Central Muon Upgrade}

Before 1992 CDF had only one central muon detector the CMU. The CMU while efficient did record occasional punch through events. Some hadronic showers continue across the 5.4 pion radiation lengths into the CMU. At the end of the 1989 run, the single muon 


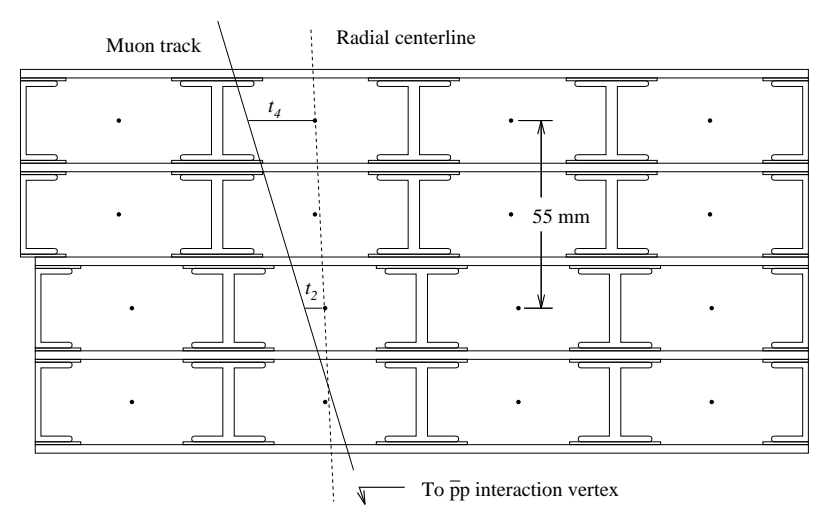

Figure 2.10: The end of a single muon module. Three of these were bolted together and attached to the back of each hadronic calorimeter tower.

trigger was dominated by hadronic punch through. The proposed way to reduce the hadronic punch though in a single high-energy muon trigger was to build the Central Muon Upgrade (CMP).

The CMP [44] was constructed around the magnetic field yoke. Each CMP chamber was built with the same cross-section as the CMU chambers: $63.5 \times 26.8 \mathrm{~mm}$ and $3200 \mathrm{~mm}$ long. These chambers were then attached to the top and bottom of the yoke. Since the yoke does not extend to cover the sides of CDF, steel walls were constructed on to which more CMP chambers could be mounted. For the two possible paths to the CMP the integral material provided eight radiation lengths.

The substructure of the steel wall and yoke CMP systems was the same, each was constructed from four layers of drift cell. Ambiguities were avoided by positioning each layer such that it was staggered with respect to the previous layer. When fully assembled the CMP chambers were filled with 50/50 Argon/Ethane. No charge separation was attempted and therefore only drift information was available, i.e. the chambers provided tracking in the plane perpendicular to the beam axis.

The CMP coverage was constrained by electrical and structural components reducing the coverage over the azimuth to $76 \%$. Within the covered azimuthal range the pseudorapidity range varies due to the mapping from the box structure to a cylinder. The coverage of the CMP detector is summarised in figure 2.9. 


\subsubsection{Central Muon Extension}

In addition to the CMP chamber upgrade the muon coverage was extended out to a pseudo-rapidity range $|\eta|<1$. The extra coverage was introduced by construction of the Central Muon Extension (CMX) detector [44].

The central muon extension was built in the form of four arches, shown in figure 2.2. Each arch contained eight 15 degree wedges, covering the range $0.6<|\eta|<1.0$ and $\Delta \phi=240$ degrees. A 30 degree gap was left at the top, to allow cryogenic fluid access for the solenoid and the main ring accelerator. Another gap was left where the conical section outlined by the arches met the floor. The gap at the floor of 90 degrees was to be covered by a "miniskirt" but remained uninstrumented during the 1992-96 running period. The coverage of these chambers was summarised in figure 2.9.

In terms of substructure, the detector was constructed from eight layers. Each arch was built from layers of rectangular drift cells of dimension $2.5 \times 15 \times 180 \mathrm{~cm}$. The arches were assembled such that successive layers were offset by half of a cell. Since each conical layer was formed from rectangular chambers, there was a resulting stereo angle of 3.6 degrees, allowing some pseudo-rapidity measurements to be made. On either side of the eight layers of drift cells a single layer of scintillator bars was added. These scintillator bars were $180 \mathrm{~cm}$ long, $2.2 \mathrm{~cm}$ thick and trapezoidal in shape: measuring $45 \mathrm{~cm}$ at one end and $27 \mathrm{~cm}$ at the other. Each scintillating bar was connected to a single photo multiplier tube. The two scintillator layers were used to select a timing gate for the drift cells.

When assembled the chambers where filled with 50/50 Argon/Ethane and operated in proportional mode. The cells were used to measure drift time only and had an azimuthal resolution in the plane perpendicular to the beam axis of $270 \mu \mathrm{m}$. Due to the position of the CMX arches the number of radiation lengths to the CMX chambers varied as a function of pseudo-rapidity: the minimum number of radiation lengths being 6 . 


\section{Chapter 3}

\section{Trigger}

\subsection{Introduction}

Every time a $p \bar{p}$ collision takes place a multitude of different particles are produced. Unlike an electron positron machine the beam particles are composite and described by parton distribution functions. Many of the interactions are soft processes, and hence are not useful for the study of heavy quarks. The overall rate of interaction without specific selection tools would swamp the available bandwidth if directly accepted to file storage. The rate at which data can be accepted is dependent on the speed at which it can be written to tape. Typically this was approximately a few $\mathrm{Hz}$ (5-8) during the Run I operational period (1992 to 1996), compared to the interaction rate of $75 \mathrm{kHz}$. To reduce the rate of accepted events a multi-stage trigger was implemented to select interesting physics events and events containing possible sources of new physics.

\subsection{General Description}

\subsubsection{Level 1}

The first two levels of trigger were designed to operate quickly, selecting a wide range of physics events. The decision time for the level 1 trigger, fixed by the speed of analogue sums and associated digitisation, was less than the $3.5 \mu$ s between bunch crossings. Therefore this trigger was always able to accept events and thus had no dead time. This was achieved by using an analogue readout, processing the associated data with dedicated FASTBUS electronics described in [3]. 
Within these stringent timing constraints, sections of the detector were examined globally. For any given trigger a number of different sections could be compared to thresholds. These sections included: summed transverse energy from hadronic and electromagnetic calorimeter elements singly or as a global sum, transverse energy imbalance, existence of stiff tracks but no directional information, muon stubs, beam-beam or beamgas interactions, and hits in small angle silicon counters in combination with beam-beam counters. Selection of interesting physics events was mostly done using calorimeter and muon chamber information exclusively. The resultant acceptance rate from this trigger was measured to be $1 \mathrm{KHz}[1]$ at an instantaneous luminosity of $5 \times 10^{30} \mathrm{~cm}^{-2} \mathrm{~s}^{-1}$.

\subsubsection{Level 2}

If an event passed the level 1 trigger logic then the analogue signals sampled were passed to the level 2 electronics. Level 2 processors resided in a single FASTBUS crate, modified by the addition of extra backplane connections. Information from the detector components was loaded into custom designed processor modules. Once the processor modules had completed their allocated tasks, (filtering muon, track or calorimeter information), the results were combined into a programmable processor card, where the level 2 accept decision was made. The combined trigger decision time was approximately $10 \mu \mathrm{s}$, implying a dead time of $5-10 \%$. During this time topological features were used to determine whether an event should be accepted or not. These features included transverse mass calculations for groups of clusters and some basic electron-pion separation. In summary the decisions were made on: the number of energy clusters and their properties, presence and position of track segments, and their matching with muon stubs, transverse energy sums and energy imbalances over the entire detector, and beam-beam or beam-gas interactions. The final output of accepted events from the level 2 trigger logic was measured to be approximately $12 \mathrm{~Hz}[1]$, at an instantaneous luminosity of $5 \times 10^{30} \mathrm{~cm}^{-2} \mathrm{~s}^{-1}$.

\subsubsection{Level 3}

Once a level 2 acceptance signal had been issued all detector information was read out and digitised taking $3 \mathrm{~ms}$. Information relating to the different components of the detector was combined into a single block of memory by the Event Builder. The buffered event was passed from the Event Builder to the level 3 system, the original configuration of which 
is described in [17]. The level 3 trigger logic was constructed entirely from software, and was upgraded to use 48 SGI RS4000 processors. Each of these processors had two buffers, to eliminate input/output time. Two buffers were present such that when operating, the level 3 trigger could accept an event into one of the processor buffers at the same time as processing filter algorithms on a previously loaded buffer. In the same manner processed events could then be streamed to tape or staging disk, while the other buffer was being considered by the trigger.

Events copied into the level 3 memory were reconstructed by the level 3 farm using an optimised form of the standard reconstruction code, building three dimensional track objects, and checking matching between muon stubs and tracks. (Silicon reconstruction was not included, trimming the processing time.) The resulting system allowed many different event types to be selected via specific filters and streamed to different tape records. Under normal operation the level 3 trigger system had no dead time except during the IA running period when two or more tapes were being changed simultaneously.

\section{$3.3 J / \psi \rightarrow \mu^{+} \mu^{-}$Trigger Path}

\subsubsection{Level 1: Muon}

At CDF there are three types of central muon chambers, described in sections 2.3.4.1, 2.3.4.2 and 2.3.4.3. All of these muon chambers measure drift times to find a single point on a track in the plane transverse to the beam axis, and therefore stacks of the chambers were used to reconstruct track segments. Since the muon chambers are outside the hadronic calorimeter steel there is little magnetic field within the chambers. The track segments reconstructed from muon chamber drift times at CDF, are therefore straight and commonly referred to as stubs. Muon stubs were reconstructed at level 1 between beam crossings and used to form crude transverse momentum constraints. Such a transverse momentum constraint was made on the slope of the stub in the plane transverse to the beam axis. Assuming no multiple scattering between the origin and the muon chambers, together with the absence of magnetic inhomogeneities in the forward region, the slope in the transverse plane could be accurately converted to a transverse momentum. However the calorimeter material and the steel return yoke did introduce multiple scattering and therefore this crude transverse momentum constraint was smeared with respect to 
momentum measurements made within the CTC.

Using the approximate transverse momentum constraint from the muon stubs, most of the dimuon data sample was accepted at level 1 through either dimuon, $3.3 \mathrm{GeV} / \mathrm{c}$ per stub or single muon, $6.0 \mathrm{GeV} / \mathrm{c}$ for one of the two stubs. The $J / \psi \rightarrow \mu^{+} \mu^{-}$level 1 sample was mainly made up of combinations of CMU-CMU or CMU-CMX chambers. Following the acceptance of the event via any one of the muon or dimuon trigger conditions, the Central Fast Tracker, described in section 3.3.2, was exercised to produce two dimensional tracks. Following two dimensional track reconstruction, tracks were matched to calorimeter and muon trigger objects.

\subsubsection{Central Fast Tracker and Associated Matching}

Muon stubs that passed the level 1 dimuon trigger constraints were matched during level 2 trigger processing to two dimensional tracks. Before this matching could take place the Central Fast Tracker (CFT) was run to reconstruct two dimensional tracks within the event.

The CFT found tracks within the CTC, described in section 2.3.2.3, using a fraction of superlayer hits. Reconstruction of a track started with a single prompt hit in superlayer 8. Prompt, implies that the track passed within $4.2 \mathrm{~mm}$ of a sense wire within a given superlayer. Since the tracking was only in two dimensions, reconstruction only involved the axial superlayers. In addition to the prompt hit, two delayed hits were searched for. Delayed hits correspond to drift times for charge carriers that traversed approximately the entire cell. Axial superlayer drift times were digitised and then discriminated within a time window as: prompt, delayed or neither. Once three hits had been found, the slope of a track segment was deduced. The speed of the tracking process was increased by using a lookup table. The values are summarised in table 3.1, used the prompt and delayed track hits to find the transverse momentum and charge within some error. Tracking of a high momentum charged particle with the CFT is illustrated in figure 3.1.

For tracks to be reconstructed by the CFT each axial layer was required to have a pair of delayed and a single prompt hit, with the possibility of one missed hit. This missed hit could be any prompt or delayed hit with the exception of superlayer 8 , where a prompt hit was required for track reconstruction to commence. The prompt hit in superlayer eight was also fundamental in the determination of the exit azimuth: key in matching the track 


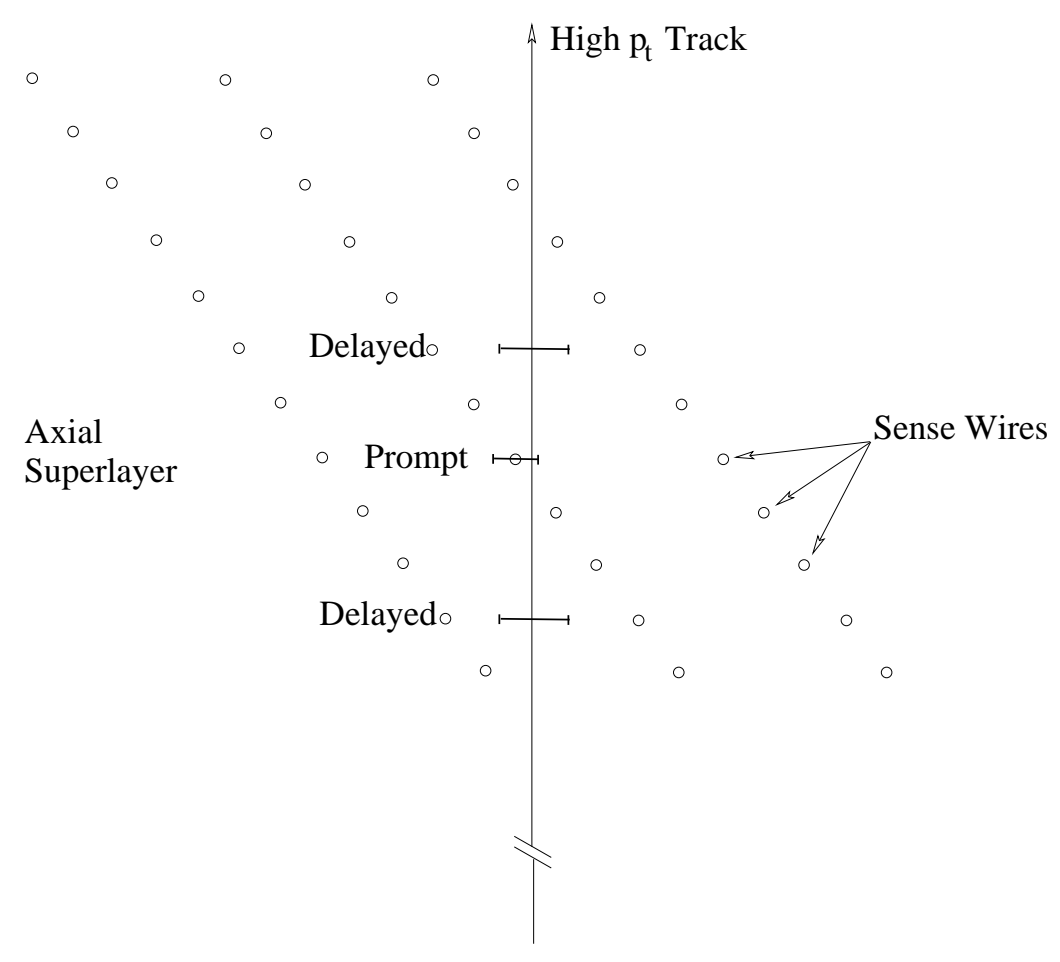

Figure 3.1: A high momentum track crossing one layer of sense wires within an axial CTC superlayer. The error bars roughly correspond to the timing windows within which prompt and delayed hits could be accepted.

to the appropriate calorimeter tower or muon chamber.

\begin{tabular}{|c|c|c|c|c|c|c|c|c|}
\hline \hline Run & \multicolumn{10}{|c|}{$p_{t}$ bin lower limit $\mathrm{GeV} / \mathrm{c}$} \\
\hline IA & 3.0 & 3.7 & 4.8 & 6.0 & 9.2 & 13.0 & 16.7 & 25.0 \\
IB & 2.2 & 2.7 & 3.4 & 4.7 & 7.5 & 12.0 & 18.0 & 27.0 \\
\hline \hline
\end{tabular}

Table 3.1: Summary of CFT $p_{t}$ binning. The table was re-optimised at the beginning of Run IB

The resultant performance of the CFT, using the lookup table values given in table 3.1, was a momentum resolution of approximately $\delta p_{t} / p_{t}=0.03 p_{t}$ where $p_{t}$ was measured in $\mathrm{GeV} / \mathrm{c}$. Besides the momentum resolution the charge determination for high energy tracks 
was not very accurate and thus opposite sign constraints were made at level 3 after three dimensional tracking.

Once a list of two dimensional tracks had been produced they were compared with calorimeter and muon trigger objects. Again to make this process as fast as possible another lookup table was implemented. For any given track the CFT $p_{t}$ and exit azimuth were used to lookup which muon or calorimeter trigger tower the two dimensional track should correspond to. During Run IA and the first part of Run 1B the muon lookup table procedure required a muon within the 15 degree allocated muon volume. This muon volume corresponded to three muon chambers in azimuth. Later in Run IB the acceptance width for the CFT to muon stubs was made wider, to encompass the effects of the higher than expected multiple scattering measured from the Run IA $J / \psi \rightarrow \mu^{+} \mu^{-}$sample.

\subsubsection{Level 2: Muon}

At level 2, dimuon triggers imposed matching between two dimensional tracks and at least one of the two level 1 muon stubs. Early data matching used a window of $\delta \phi<15$ degrees within which to search for stubs, though this was later reduced to 5 degrees to reduce accidental coincidences. Secondly during Run IB the trigger was modified to accept only dimuons that were both matched to CFT tracks. In summary the main level 2 dimuon triggers involved two CMU stubs each matching a CFT track during Run IB, or two CMU stubs where only one had a matching CFT track during Run IA.

\subsubsection{Level 3: $J / \psi \rightarrow \mu^{+} \mu^{-}$}

At level 3 tracks within the CTC and VTX were reconstructed in three dimensions, by running an optimised version of the offline reconstruction software. These tracks were then matched with reconstructed muon stubs. For an event to pass the level $3 \mathrm{~J} / \psi \rightarrow \mu^{+} \mu^{-}$ trigger it had to pass track matching constraints between stubs and helical tracks together with a mass constraint on the reconstructed $J / \psi$, given in table 3.2 .

The level 3 trigger accepted two streams of $J / \psi \rightarrow \mu^{+} \mu^{-}$events denoted A and B. Stream A $J / \psi$ events passed either a single or dimuon level 2 trigger, and then passed the level 3 constraints. Stream B events passed any level 2 trigger and then passed the level 3 constraints for $J / \psi \rightarrow \mu^{+} \mu^{-}$. The difference between B and A was found to be of the order of $1 \%$, explained by fake muons allowing acceptance of most of the potential 


\begin{tabular}{|c|c|c|}
\hline \hline Run & Mass Window & Stub to Track Acceptance \\
\hline \hline IA & 2.8 to $3.4 \mathrm{GeV} / \mathrm{c}^{2}$ & $4 \sigma$ \\
\hline IB & 2.7 to $4.1 \mathrm{GeV} / \mathrm{c}^{2}$ & $3 \sigma$ \\
\hline \hline
\end{tabular}

Table 3.2: Level $3 J / \psi \rightarrow \mu^{+} \mu^{-}$trigger constraints during the Run I operational period.

stream B only events into the stream A dataset [51]. Though the difference was found to be small, the stream B $J / \psi$ data were used to insure all $J / \psi$ candidates were available.

\subsubsection{Trigger Summary}

A summary of the main Run Ib $J / \psi \rightarrow \mu^{+} \mu^{-}$triggers is given in table 3.3. The three triggers all require two CFT tracks within the first bin given in table 3.1. These CFT tracks must also be matched to muon chamber stubs. The TWO-CMU implies two CMU chamber stubs have been matched to two tracks; CMX-CMU one track has been matched to a CMX chamber stub and one to a CMU chamber stub; and TWO-CMX two CMX chamber stubs have been matched to two tracks. All of these triggers require level 1 muon triggering of the given stub type as described in section 3.3.1.

\begin{tabular}{|c|c|}
\hline \hline Trigger Name & Fraction (\%) \\
\hline \hline TWO-CMU-TWO-CFT-2-2 & $58.52 \pm 0.20$ \\
\hline CMX-CMU-TWO-CFT-2-2 & $18.45 \pm 0.11$ \\
\hline TWO-CMX-TWO-CFT-2-2 & $2.06 \pm 0.04$ \\
\hline
\end{tabular}

Table 3.3: A summary of the main $J / \psi$ level 2 triggers during the Run Ib running period taken from [49]. 


\section{Chapter 4}

\section{Analysis}

\subsection{Introduction}

This chapter describes the analysis which was carried out with the Collider Detector at Fermilab (CDF). At the time of writing only a branching ratio limit for the decay $B_{s}^{0} \rightarrow J / \psi \eta$ exists. The limit published by the L3 collaboration [2], is $\mathcal{B}\left(B_{s}^{0} \rightarrow J / \psi \eta\right)<$ $3.8 \times 10^{-3}$ at a $90 \%$ confidence level. To obtain this limit the production fractions were assumed to be $f_{u}=39.5 \pm 4.0 \%$ and $f_{s}=12.0 \pm 3.0 \%$.

In light of the current status of the $B_{s}^{0} \rightarrow J / \psi \eta$ branching ratio, the main objective of this analysis was to find evidence for the $B_{s}^{0} \rightarrow J / \psi \eta$ decay within recorded CDF data. From these data a branching ratio limit was determined. This branching ratio was not measured directly but through a ratio of ratios, to minimise the effect of systematic errors connected with the $J / \psi$ sample. $B^{ \pm} \rightarrow J / \psi K^{ \pm}$was an obvious candidate for the other half of the ratio of ratios since the systematics connected with the $K^{ \pm}$have previously been extensively studied by the CDF collaboration [40][39].

The analysis is described starting from the simulation used to calculate acceptance effects. Then the data analysis used to search for the $B_{s}^{0} \rightarrow J / \psi \eta$ decay and the analysis of $B^{ \pm} \rightarrow J / \psi K^{ \pm}$from data is given in detail. Following the data analysis, studies of calorimeter efficiencies are described. 


\subsection{Simulation}

\subsubsection{Introduction}

The simulation described in the following sections was written to calculate the ratio of acceptances between the two signals $B_{s}^{0} \rightarrow J / \psi \eta$ and $B^{ \pm} \rightarrow J / \psi K^{ \pm}$. These acceptances were calculated by generating

$$
\begin{aligned}
& B^{ \pm} \rightarrow J / \psi \quad K^{ \pm} \\
& \hookrightarrow \mu^{+} \mu^{-}
\end{aligned}
$$

and

$$
\begin{array}{ccc}
B_{s}^{0} \rightarrow \quad J / \psi & \eta \\
& \hookrightarrow \gamma \gamma \\
& \hookrightarrow \quad \mu^{+} \mu^{-}
\end{array}
$$

events. A primary vertex position was generated from a histogram of primary vertices taken from $J / \psi \rightarrow \mu^{+} \mu^{-}$data. Muon daughters from the $J / \psi$ were projected into the detector so that geometrical and trigger constraints could be made. The resulting $B_{s}^{0}$ and $B^{ \pm}$events were then treated according to the geometrical acceptance and detector reconstruction efficiency associated with the $\eta \rightarrow \gamma \gamma$ decay and the $K^{ \pm}$daughter. Finally, from initially equal numbers of $B_{s}^{0}$ and $B^{ \pm}$events the ratio of the acceptances was calculated by multiplying by $f_{s} / f_{d}$.

\subsubsection{B Meson Production}

The $B^{ \pm} \rightarrow J / \psi K^{ \pm}$and $B_{s}^{0} \rightarrow J / \psi \eta$ decays were modelled without any underlying event. ${ }^{1}$ Both $B_{s}^{0}$ and $B^{ \pm}$mesons were generated using a theoretical cross-section distribution together with Peterson fragmentation, producing the selected meson state.

Before the simulation could run the inclusive bottom quark cross-section as a function of $p_{t}$ and rapidity was calculated theoretically. This calculation described in [45] uses next to leading order (NLO) QCD, and was performed using parameters associated with the Tevatron environment: $p \bar{p}$ at $1.8 \mathrm{TeV}$. Results from the NLO QCD calculation were packed into a two dimensional histogram. The NLO QCD calculation package used, provides an interface to allow parton distribution functions (PDFs) for the proton to be chosen. Previously Tevatron b-physics cross-section results have been compared to calculations

\footnotetext{
${ }^{1}$ The effects of the underlying event on the photon reconstruction are discussed later in this chapter.
} 
performed with the MSRD0 [46] PDF. This analysis uses the updated PDF MRST991 [47], which takes into account the latest fits from HERA. A comparison of the MRSD0 and MRST991 PDFs was made with CDF data in figure 4.1.

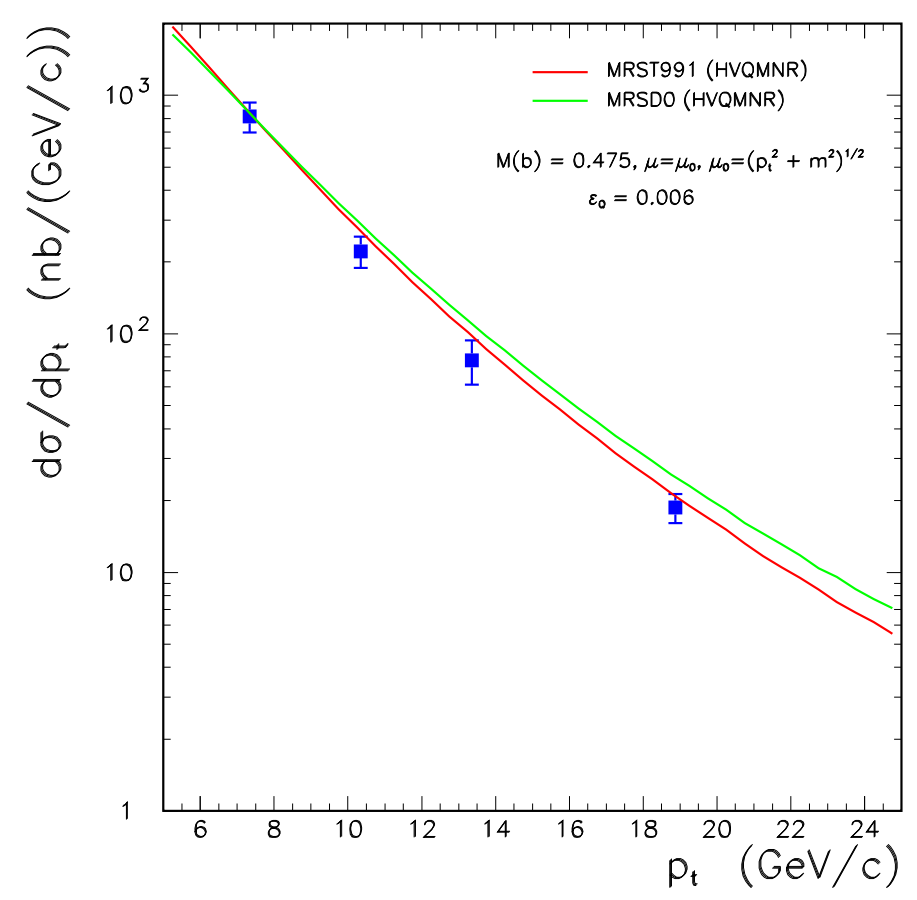

Figure 4.1: Renormalised NLO QCD cross-sections for $B^{ \pm}$production compared with CDF data[18]. B mesons were generated using Peterson fragmentation. The theoretical curves have been renormalised in an attempt to fit the data.

Once the b quark cross-section had been calculated using NLO QCD and MRST991 the output histogram was used to produce b quark events with a generated $p_{t}$ and rapidity. To produce b quark four-vectors, the azimuthal angle $\phi$ of the b quark was selected with a flat distribution between 0 and $2 \pi$. The transverse momentum, rapidity and the angle $\phi$ were then substituted into equation 4.1, to produce a four-vector.

$$
\begin{gathered}
p_{x}=p_{t} \cos (\phi) \\
p_{y}=p_{t} \sin (\phi) \\
p_{z}=\sqrt{\frac{m_{b}^{2}+p_{t}^{2}}{\alpha}} \\
E=\sqrt{m_{b}^{2}+p_{t}^{2}+p_{z}^{2}}
\end{gathered}
$$


where

$$
\alpha=\left(\frac{e^{2 y}+1}{e^{2 y}-1}\right)^{2}-1,
$$

$p_{t}$ is the transverse momentum, $y$ is the rapidity, and $m_{b}$ is the bare mass of the b quark.

As previously mentioned B mesons were generated by using the Peterson fragmentation model.

\subsubsection{Modelling Fragmentation}

Fragmentation describes the process of confinement. In a $p \bar{p}$ collision single heavy quarks may be produced. These heavy quarks quickly become bound inside mesons or baryons. Since the process of confinement is at the scale of the mass of the resultant meson, $\alpha_{s}$ is very close to one. This means that the use of QCD to describe the process with traditional perturbation theory has large errors associated with it. For the purpose of this thesis the Peterson fragmentation model, given in [50], was used to produce a $p_{t}$ spectrum of b quark mesons:

$$
D_{Q}^{H}(z)=\frac{N}{z\left(1-\frac{1}{z}-\frac{\epsilon_{Q}}{1-z}\right)^{2}}
$$

where:

$$
z=\frac{\left(E+p_{/ /}\right)_{Q \bar{q}}}{E+p_{Q}}
$$

$z$ is the fraction of the momentum that the final hadron has in the direction of the original heavy quark. This can be simply expressed as: $z=p_{\|}^{H} / p_{\|}^{Q}$. The behaviour of this function for an arbitrary normalisation is illustrated in figure 4.2.

The Peterson fragmentation model is a phenomenological model, i.e. measured to fit the data with no knowledge of the internal physics. It describes the amount of momentum parallel to the $\mathrm{b}$ quark direction that the resultant hadron inherits from the $\mathrm{b}$ quark. The model is only valid for heavy quarks charm and bottom, and the value of $\epsilon_{Q}$ is normally chosen via a fit to the data. A detailed discussion of different phenomenological models and attempts to use perturbative QCD can be found in [25].

\subsection{3 $\quad B^{+}$and $B_{s}^{0}$ Meson Decays}

$\mathrm{B}$ mesons were produced using the methods discussed in section 4.2.2. Following fragmentation of the generated b quark into either a $B^{+}$or $B_{s}^{0}$ meson, daughters for the specific decay were produced. The energy of the first daughter in the rest frame of the decaying 


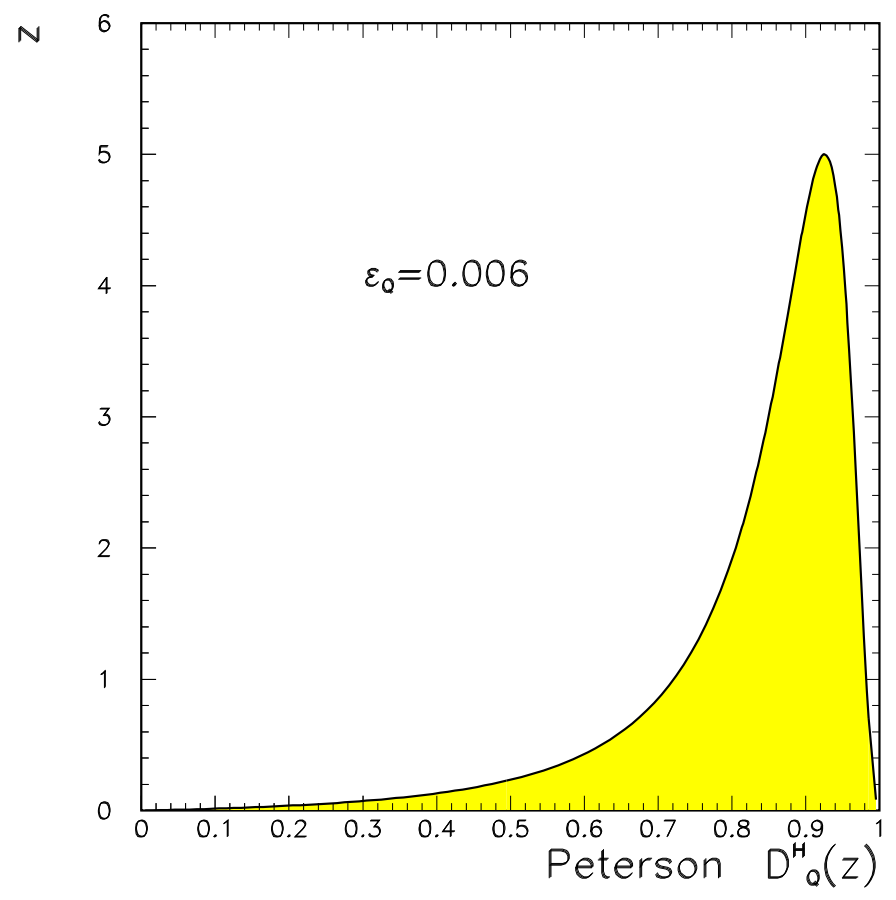

Figure 4.2: The Peterson fragmentation function for $\epsilon_{Q}=0.006$, the value used for $b$ quark decays at CDF.

meson, was calculated from equation 4.2. Then using energy conservation the energy of the other daughter particle was found, and hence both momenta.

$$
E_{1}=\frac{M^{2}+m_{1}^{2}-m_{2}^{2}}{2 M}
$$

where $E_{1}$ is the energy of the first daughter, $m_{1}$ and $m_{2}$ refer to the masses of the daughter particles, and $\mathrm{M}$ is the mass of the decaying particle.

To use the momenta and energy to generate four-vectors, $\cot (\theta)$ and $\phi$, were picked using flat distributions between $(0,1)$ and $(0,2 \pi)$. These values were then used to calculate the components of the first daughter. From this the second daughter was then inferred from momentum conservation. Both daughters were boosted back into the lab frame using a Lorentz transformation. 


\subsubsection{Constraints on $J / \psi \rightarrow \mu^{+} \mu^{-}$}

The Monte Carlo methods described in sections 4.2.2 and 4.2.3, were used to generate two samples of $10^{8}$ mesons decaying into each respective decay channel. During both Monte Carlo runs, a primary vertex position was generated using methods discussed in section A.1. Then all the tracks were projected into the CDF geometry. By procedures described in section A.2, the $\mu^{ \pm}$daughters of both decay channels were required to be in either the CMU or CMX detectors. These central muon chambers were selected in preference to the forward chambers because the tracking resolution in the central region was good enough to allow separation of prompt charm $J / \psi$ 's from b daughters. Muon pairs found to be within the geometric limits of the CMU and CMX detectors were required to pass both level 1 and level 2 dimuon triggers: previously discussed in chapter 3 . The details of implementing these trigger acceptances are given in sections A.3 and A.4. Specifically the muon pairs were required to pass one of the triggers described in section 3.3.5.

\subsubsection{Constraints on $K^{ \pm}$}

$B^{ \pm} \rightarrow J / \psi K^{ \pm}$decays were produced with the methods described in sections 4.2.2 and 4.2.3. These events were then required to pass the $J / \psi \rightarrow \mu^{+} \mu^{-}$geometrical and trigger constraints described in section 4.2.4. Each Monte Carlo event was then analysed using the same strategy as later applied to CDF data.

The analysis of $B^{ \pm} \rightarrow J / \psi K^{ \pm}$within both Monte Carlo and data followed the prescription in [39]. Within this previous work kaons were required to have a transverse momentum greater than $1.25 \mathrm{GeV} / \mathrm{c}$. The exit radius from the CTC tracking volume was required to be $110 \mathrm{~cm}$ or more, to ensure good reconstruction efficiencies. Then the track reconstruction efficiency was applied using figure 4.8. Constraints made on the generated kaon are summarised in table 4.1.

\begin{tabular}{|c|c|}
\hline \hline Description & Constraint \\
\hline Kaon $p_{t}$ & $\geq 1.25 \mathrm{GeV} / \mathrm{c}$ \\
Kaon CTC Exit Radius & $\geq 110 \mathrm{~cm}$ \\
\hline \hline
\end{tabular}

Table 4.1: Constraints made on the generated $K^{ \pm}$particle from the decay $B^{ \pm} \rightarrow J / \psi K^{ \pm}$. 
The constraints given in table 4.1 were made on an event by event basis. The effect of the vertex $\chi^{2}$ cut made on the data sample and the CTC tracking efficiency were applied by multiplying the final number of events reconstructed by the respective efficiency factors. A discussion of these values is given in section 4.4.2.

\subsubsection{Constraints on $\eta \rightarrow \gamma \gamma$}

$B_{s}^{0}$ mesons were generated with the same methods as $B^{ \pm}$mesons. $B_{s}^{0} \rightarrow J / \psi \eta$, $J / \psi \rightarrow \mu^{+} \mu^{-}, \eta \rightarrow \gamma \gamma$ decays were produced. $J / \psi$ muon daughters were required to pass the trigger constraints described in section 4.2.4. Photon daughters, from surviving $B_{s}^{0} \rightarrow J / \psi \eta$ events, were projected into the calorimetry geometry. For photons within the CEM, the generated $E_{t}$ was required to be greater than $0.8 \mathrm{GeV}$. The choice of this constraint is discussed at length in appendix C. Then using the generated primary vertex position both photons were required to be within towers (0-8): corresponding to \pm 1 unit of pseudorapidity from the centre of the detector. Both photons were required not to be within the chimney tower since the tower has poor energy resolution and no CES chamber within it. Both photons were required to be within the CES volume at the CES depth, because the CES does not stretch the entire length of the CEM. Then the Monte Carlo photon daughters were required to be within two wire and two strip cells of the CES half chamber boundaries, ensuring that CES clustering might function.

For photon pairs passing all geometric constraints the CES reconstruction process was simulated. The simulation of the CES reconstruction was done by using the energy of each photon to calculate the probability of its reconstruction. Once the probability had been calculated the CES detector efficiency was applied using a random number generator. The calculation of the CES reconstruction efficiency is described in section 4.4.3. The detector efficiency had to be applied on an event by event basis because it is a function of $E_{t}$ for low energy photons.

The mass difference $M(J / \psi \eta)-M\left(B_{s}^{0}\right) \quad$ Using the CDF detector the measured width of the $B_{s}^{0}$ mass from the decay $B_{s}^{0} \rightarrow J / \psi \eta$ is expected to be heavily dependent on the central electromagnetic calorimeter resolution. Using this assumption the width $\Gamma\left(B_{s}^{0}\right)$ was reconstructed from Monte Carlo. Photon daughters from the generated decays were smeared according to the measured resolution $\sigma(E) / E$ of $13.5 \% / \sqrt{E}[9]$. Then the $B_{s}^{0}$ mass 

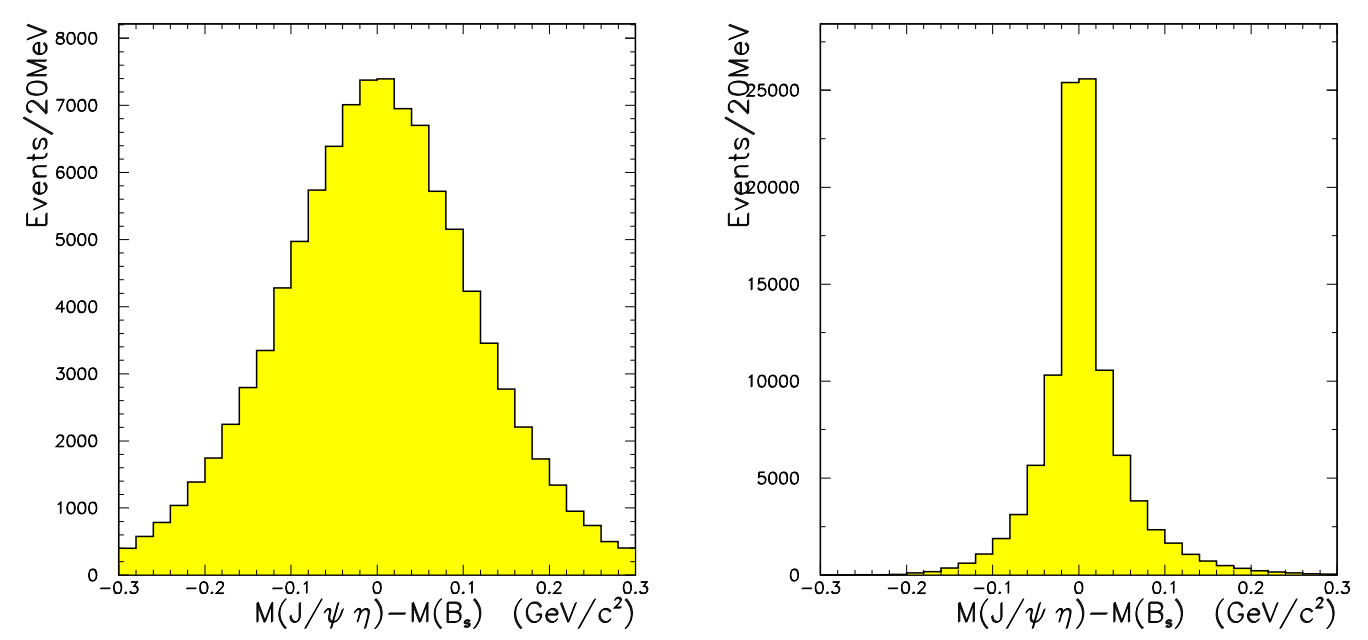

Figure 4.3: A comparison of $M(J / \psi \eta)-M\left(B_{s}^{0}\right)$ assuming the width is only dependent on the electromagnetic calorimeter resolution. Left and right plots correspond to the corrected (equation 4.3) and uncorrected mass distributions.

was recalculated using the smeared photons. During the analysis of data the smearing was corrected for by pulling the mass to that of the $\eta$. This was deemed acceptable since the kinematic mass width $\Gamma(\eta)=1.18 \pm 0.11 \mathrm{keV}$ is small in comparision to the experimentally measured one. The correction for the effects of energy resolution was done by altering the combined photon four-vector by the amount their invariant mass deviated from the world average $\eta$ mass. This can be simply expressed as written in equation 4.3.

$$
\overrightarrow{B_{s}^{0}}=\overrightarrow{J / \psi}+\frac{m_{\eta}^{P D G}}{m_{\eta}^{\text {meas }}}\left(\overrightarrow{\gamma_{1}}+\overrightarrow{\gamma_{2}}\right)
$$

where $\overrightarrow{B_{s}^{0}}, \vec{J} / \psi, \vec{\gamma}$ refer to the four-vector quantities describing the respective particles, $m_{\eta}^{P D G}$ is the world average $\eta$ mass, and $m_{\eta}^{\text {meas }}$ is the measured $\eta$ mass.

From the generated Monte Carlo decays the $B_{s}^{0}$ mass was reconstructed before and after applying equation 4.3. The comparison between the uncorrected and corrected $B_{s}^{0}$ mass distributions is shown in figure 4.3 . 


\subsubsection{Calculating the expected number of $B_{s}^{0} \rightarrow J / \psi \eta$ events}

The number of $B_{s}^{0} \rightarrow J / \psi \eta$ events was predicted by using the $B^{ \pm} \rightarrow J / \psi \eta$ and $B_{s}^{0} \rightarrow J / \psi \eta$ Monte Carlo samples. Each of these samples was subject to all trigger, geometrical and detector-related reconstruction efficiencies. The resulting number of events from $10^{8}$ meson decays was used together with equation 4.4 to calculate a Run I prediction.

$$
N^{o}(J / \psi \eta) \approx \frac{f_{s}}{f_{u}} \frac{\mathcal{B}\left(B_{s}^{0} \rightarrow J / \psi \eta\right) \mathcal{B}(\eta \rightarrow \gamma \gamma)}{\mathcal{B}\left(B^{+} \rightarrow J / \psi K^{+}\right)} \frac{M C(J / \psi \eta)}{M C\left(J / \psi K^{+}\right)} N^{o}\left(J / \psi K^{+}\right)
$$

where $f_{s} / f_{u}$ is the ratio of the fragmentation fractions, $\mathcal{B}\left(B_{s}^{0} \rightarrow J / \psi \eta\right)$ is an input branching ratio, $\mathcal{B}(\eta \rightarrow \gamma \gamma)$ and $\mathcal{B}\left(B^{+} \rightarrow J / \psi K^{+}\right)$are measured branching ratios, $M C(J / \psi \eta)$ is the number of simulation events passing simple constraints, and $N^{o}\left(J / \psi K^{+}\right)$ is the number of $B^{+} \rightarrow J / \psi K^{+}$observed from analysis of the run I CDF data.

Approximating $\mathcal{B}\left(B_{s}^{0} \rightarrow J / \psi \eta\right)$ From equation 4.4 values of all variables are known with associated errors except the branching ratio $\mathcal{B}\left(B_{s}^{0} \rightarrow J / \psi \eta\right)$ which is the subject of this thesis. In order that a prediction of the number of $B_{s}^{0} \rightarrow J / \psi \eta$ events could be made, the branching ratio was estimated by using $B_{s}^{0} \rightarrow J / \psi \phi$.

The decay $B_{s}^{0} \rightarrow J / \psi \phi$ is described by the same Feynmann diagram as $B_{s}^{0} \rightarrow J / \psi \eta$, illustrated in figure 4.4. Loosely speaking the $\eta$ meson only differs from the $\phi$ meson by quark content and spin. The quark model for the daughter particles is summarised in table 4.2 .

\begin{tabular}{|c|c|c|c|c|}
\hline \hline Particle & Quark Content & Spin & Parity & Charge Conjugation \\
\hline$\eta$ & $\frac{1}{2}(u \bar{u}+d \bar{d})-\frac{1}{\sqrt{2}} s \bar{s}$ & 0 & odd & even \\
$J / \psi$ & $c \bar{c}$ & 1 & odd & odd \\
$\phi$ & $s \bar{s}$ & 1 & odd & odd \\
\hline \hline
\end{tabular}

Table 4.2: Meson Particle Properties from the Particle Data Book [33].

Incorporating the reduced $s \bar{s}$ content and reduction in final spin states. The branching ratio $\mathcal{B}\left(B_{s}^{0} \rightarrow J / \psi \eta\right)$ was approximated as:

$$
\mathcal{B}\left(B_{s}^{0} \rightarrow J / \psi \eta\right) \approx \frac{1}{3 \sqrt{2}} \mathcal{B}\left(B_{s}^{0} \rightarrow J / \psi \phi\right)
$$




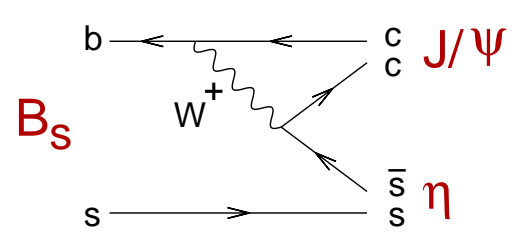

Figure 4.4: Decay of $B_{s}^{0} \rightarrow J / \psi \eta$ or $B_{s}^{0} \rightarrow J / \psi \phi$

Substituting $\mathcal{B}\left(B_{s}^{0} \rightarrow J / \psi \phi\right)$ from the $\mathrm{PDG}[33]$ into equation 4.5 the approximation predicts $\mathcal{B}\left(B_{s}^{0} \rightarrow J / \psi \eta\right) \simeq(2.2 \pm 0.8) \times 10^{-4}$ where the given error does not contain the theoretical uncertainties, but was calculated from the $\mathcal{B}\left(B_{s}^{0} \rightarrow J / \psi \phi\right)$ measurement error.

\subsection{Analysis of CDF data}

\subsubsection{Introduction}

The CDF detector collected a large sample of $J / \psi \rightarrow \mu^{+} \mu^{-}$decays. These $J / \psi$ particles were an inclusive sample i.e. all possible decays resulting in a $J / \psi$ daughter passing trigger constraints were contained. The same sample was used for both the repeated $B^{ \pm} \rightarrow J / \psi K^{ \pm}$analysis and the $B_{s}^{0} \rightarrow J / \psi \eta$ analysis.

Each decay channel was investigated with the constraints also applied to the generated Monte Carlo samples. A final number of $B^{ \pm} \rightarrow J / \psi K^{ \pm}$decays was found and a limit on the number of $B_{s}^{0} \rightarrow J / \psi \eta$ events was calculated.

\subsubsection{Selecting a $J / \psi \rightarrow \mu^{+} \mu^{-}$sample}

During the run I data taking period of $1992-1995,110 \mathrm{pb}^{-1}$ of $J / \psi \rightarrow \mu^{+} \mu^{-}$data was accumulated. These data were collected through the stream B level 3 trigger described in chapter 3. In the search for both decays all possible muon pairs were considered within each event. Matching between helical tracks and muon stubs was used to remove unwanted particles and noise from the muon chambers, reducing the background under the $J / \psi$ signal.

In each $J / \psi$ event muon stubs were formed inside two of the stacked CMU, CMX, and CMP drift chambers, previously discussed in section 2.3.4. These muon stubs were described within a local wedge coordinate system where the $y$-axis pointed radially outwards, the $z$-axis was aligned along the global $z$ of the beam line and the $x$-axis pointed 
according to a right handed coordinate system. Within this coordinate system $x=0$ was defined to be in middle of a 15 degree detector wedge, and $y=0$ was set as the inner surface of the muon detector in question. For a set of hits in a wedge, two lines were fitted with a polynomial of the form $f(y)=a y+c$ : one in the $x y$ plane, $\left(x=S_{x y} \times y+I_{x y}\right)$ and one in the $z y$ plane, $\left(z=S_{z y} \times y+I_{z y}\right)$.

The accuracy of the match between helical track and muon chamber stub was measured by projecting the candidate muon track from the CTC into the muon chambers. In a muon chamber the extrapolation of a helical track is straight and in the same manner as the muon stub, made up of two components. Taking the two components from the muon chamber fit, the difference between the intercepts and the gradients were calculated. From these values and the correlation coefficient, the $\chi^{2}$ terms of equations 4.6 and 4.7 were derived in [29]. Within this derivation, matching between muon stubs and helical tracks was assumed to be dominated by multiple scattering in the calorimeter and steel of the magnetic field yoke.

$$
\begin{aligned}
& \chi_{x}^{2}=\left(\begin{array}{ll}
\delta I_{x y} & \delta S_{x y}
\end{array}\right)\left(\begin{array}{cc}
\sigma_{I x}^{2} & \gamma_{x} \\
\gamma_{x} & \sigma_{S x}^{2}
\end{array}\right)^{-1}\left(\begin{array}{c}
\delta I_{x y} \\
\delta S_{x y}
\end{array}\right) \\
& \chi_{z}^{2}=\left(\begin{array}{ll}
\delta I_{z y} & \delta S_{z y}
\end{array}\right)\left(\begin{array}{cc}
\sigma_{I z}^{2} & \gamma_{z} \\
\gamma_{z} & \sigma_{S z}^{2}
\end{array}\right)^{-1}\left(\begin{array}{c}
\delta I_{z y} \\
\delta S_{z y}
\end{array}\right)
\end{aligned}
$$

where $\delta I$ corresponds to the difference between the two intercepts, $\delta S$ refers to the difference between the two slopes, and $\gamma$ contains the correlation information, defined such that: $\gamma /\left(\sigma_{I} \sigma_{S}\right)$ is the correlation coefficient.

The muon matching constraints between the chambers and the helical tracks were set to be the same for all chamber types: CMU, CMX and CMP. In the local $x$ direction the equation 4.6 was required to be less than 9 , where equation 4.6 is a $\chi^{2}$ with two degrees of freedom. In contrast the value of equation 4.7 was required to be less than 12 for CMU and CMX matches. (The CMP does not provide detailed information of muon stub position with respect to the $z$-axis.) The $\chi^{2}$ constraint along the $z$-axis was increased with respect to the $x y$ plane to take into account the effect of the poor central tracking resolution in that direction.

Muon pairs passing the $\chi^{2}$ matching constraints between track and stub were required to be opposite charge. The primary vertex was then defined in the plane perpendicular to 


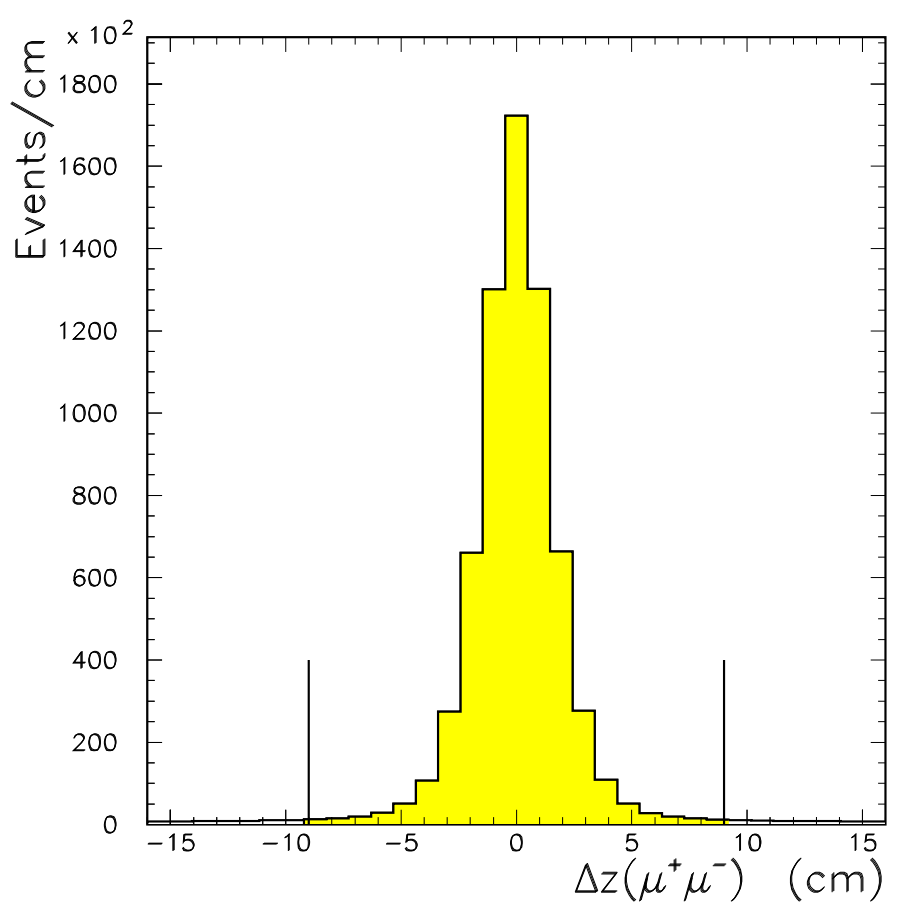

Figure 4.5: The distribution of muon pair primary vertices with respect to each other, where $\Delta z_{0}=z 1_{0}-z 2_{0}$. The selected region is marked with vertical lines and shaded yellow.

the beam line by the beam position measurement and the mean position of the two muons along the z-axis. $\mu^{+} \mu^{-}$pairs were selected with $\Delta z_{0}<9 \mathrm{~cm}$, where $z_{0}$ is the distance of closest approach to the z-axis illustrated in appendix $\mathrm{E}$. The distribution of $\Delta z_{0}$ and the selected range is illustrated in figure 4.5.

The tracking resolution along the $z$-axis was fixed at $\sigma_{z}=0.3 \mathrm{~cm}$. This number was found from the observed resolution of VTX and was fixed because the measurements given on an event by event basis from the fitting software was found to be unreliable. The poor vertex resolution along the beam axis encompasses the decay time of a $\mathrm{B}$ meson and therefore all lifetime information was only calculated in the transverse plane.

Muons passing the track and stub matching requirements, were used to form a $J / \psi$ candidate mass. The mass distribution is plotted in figure 4.6. A $75 \mathrm{MeV}$ mass window either side of the world average $J / \psi$ mass was used to exclude most of the side bands. Finally to ensure good secondary vertex resolution in the plane perpendicular to the $z$ - 

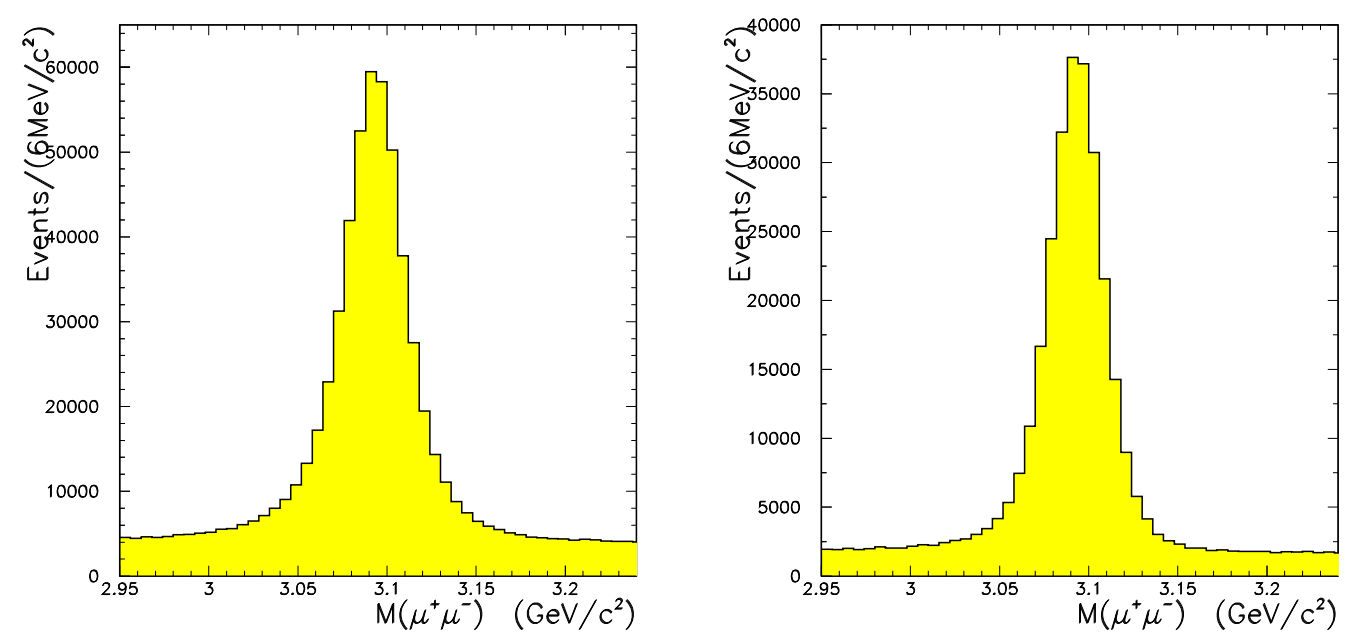

Figure 4.6: The invariant mass distribution for two opposite sign muons both passing matching constraints between track and muon stub. Left without SVX requirements, Right requiring three or more SVX hits for each muon daughter.

axis both muon tracks were required to be attached to three or more layers of the SVX detector. The resultant distribution for the primary vertex along the $z$-axis is illustrated in figure A.1.

\begin{tabular}{|c|c|}
\hline \hline Description & Constraint \\
\hline Stub to track $\chi_{x}^{2}$ & $<9$ \\
Stub to track $\chi_{z}^{2}$ & $<12$ \\
Muon pair $\Delta z_{0}$ & $<9 \mathrm{~cm}$ \\
$\left|m\left(\mu^{+} \mu^{-}\right)-m(J / \psi)\right|$ & $<75 \mathrm{MeV}$ \\
\hline \hline
\end{tabular}

Table 4.3: $J / \psi$ selection criterion made on the $J / \psi$ stream B sample

\subsubsection{Finding $B^{ \pm} \rightarrow J / \psi K^{ \pm}$}

The search for $B^{ \pm} \rightarrow J / \psi K^{ \pm}$decays started with the $J / \psi$ sample described in section 4.3.2 and then followed the recipe used in [18]. During the $B^{ \pm} \rightarrow J / \psi K^{ \pm}$analysis outlined within [18], care was taken to study all of the uncertainties involved. For the purpose of the analysis contained in this thesis the systematic errors previously measured from the 
study of $B^{ \pm} \rightarrow J / \psi K^{ \pm}$were used to calculate final uncertainties.

Starting from muon pairs passing $J / \psi$ selection criterion, discussed in section 4.3.2, all tracks which had not been identified as muons were considered to be either $K^{+}$or $K^{-}$mesons. Each candidate kaon track was required to be reconstructed from 2 or more axial layers with 4 or more used hits, and 2 or more stereo layers with 2 or more used hits. These minimal track quality constraints removed poorly reconstructed tracks. $K^{ \pm}$ candidates were accepted if they had a CTC exit radius of $110 \mathrm{~cm}$ or more. Kaon candidates passing the track quality constraints were required to have transverse momentum of at least $1.25 \mathrm{GeV} / \mathrm{c}$. This $p_{t}$ threshold ensured all kaons were within the plateau region of the CTC reconstruction efficiency curve discussed in section 4.4.2, and helped to reduce combinatorial backgrounds.

For each selected pairs of muon tracks, the kaon candidate was combined to form a $B^{ \pm}$candidate. The $B^{ \pm}$was required to have a transverse momentum above $6.0 \mathrm{GeV} / \mathrm{c}$, reducing backgrounds. (Low transverse energy $B^{ \pm}$mesons cannot be reconstructed accurately because there is insufficient energy to boost the $\mu^{+} \mu^{-}$, and $K^{ \pm}$such that they can all be accepted.) The two muon tracks and the kaon candidate were then constrained to come from the same point. This was done using a vertex $\chi^{2}$ from the differences between the mean impact parameter and the two components in the plane perpendicular to the z-axis. The two-dimensional vertex $\chi^{2}$ has $(N-2)$ degrees of freedom where $N$ is the number of tracks in the vertex. Vertices were required to form a $\chi^{2}$ less than 10 , with a corresponding probability of $99.8 \%$, and have a proper lifetime $\tau_{\text {true }}$, such that $c \tau_{\text {true }}>100 \mu \mathrm{m}$. The true proper time is related to the displacement of the secondary vertex, as stated in equation 4.8 .

$$
\lambda^{B} \equiv c \tau_{\text {true }}=L_{x y}^{B} \frac{m_{B}}{p_{t}^{B}},
$$

where $m_{B}$ is the mass of the $\mathrm{B}$ meson, $p_{t}^{B}$ is the transverse momentum of the $\mathrm{B}$ meson and $L_{x y}^{B}$, is the displacement of the secondary vertex from the primary vertex within the transverse plane, described fully in appendix E.

\subsubsection{Search for $B_{s}^{0} \rightarrow J / \psi \eta$}

Starting again from muon pairs passing requirements given in section 4.3.2, photon candidate towers were selected by searching in a 1.6 radian cone around the $J / \psi$ four- 


\begin{tabular}{|c|c|}
\hline \hline Description & Constraint \\
\hline Kaon $p_{t}$ & $\geq 1.25 \mathrm{GeV} / \mathrm{c}$ \\
Kaon CTC Exit Radius & $\geq 110 \mathrm{~cm}$ \\
$K^{ \pm} \mu^{+} \mu^{-}$vertex $\chi^{2}$ & $<10$ \\
$p_{t}\left(B^{ \pm}\right)$ & $>6.0 \mathrm{GeV}$ \\
$c \tau_{\text {true }}$ & $>100 \mu \mathrm{m}$ \\
\hline \hline
\end{tabular}

Table 4.4: A summary of the constraints made on the kaon candidate and the reconstructed $B^{ \pm}$meson.

vector. Each candidate tower was required to have no charged tracks entering or present at the CES depth, a total electromagnetic energy above $0.8 \mathrm{GeV}$, and a ratio of hadronic over electromagnetic energy less than 0.05 .

Calorimeter wedges containing one or more photon candidate towers were selected for CES reconstruction. The reconstruction process is described in appendix B. This resulted in a fitted shower centroid within the CES chamber, at the depth of approximately six radiation lengths. For a photon pair to be considered both photons were required to have reconstructed centroids within the CES. The position of the electromagnetic shower within the CES was used together with the $J / \psi \rightarrow \mu^{+} \mu^{-}$vertex, and the energy measurement in the central calorimeter to form photon four-vectors. After a list of photon four-vectors had been accumulated, each possible pair was combined with the $J / \psi$ four-vector forming candidate $B_{s}^{0}$ mesons. These candidates were retained if the two photons were found to be in a different wedge or in two different towers, and reconstructed within $120 \mathrm{MeV}$ of the world average $\eta$ meson mass. The chimney tower was excluded, and tower 9 was excluded. Tower 9 was not used because it has poor electromagnetic and hadronic energy resolution, whereas the chimney tower was ignored because the CES chamber in that wedge does not extend into the chimney tower. $B_{s}^{0}$ meson candidates passing these constraints were required to have a $p_{t}$ above $6.0 \mathrm{GeV} / \mathrm{c}$, and an approximate lifetime $\tau_{\text {approx }}$ such that $c \tau_{\text {approx }}$ was greater than $100 \mu \mathrm{m}$. Again the lifetime was expressed in terms of the secondary vertex displacement:

$$
\lambda^{B} \approx c \tau_{\text {approx }}=\frac{\lambda^{J / \psi}}{f\left(p_{t}^{J / \psi}\right)}=\frac{L_{x y}^{J / \psi}}{(\beta \gamma)_{t}^{J / \psi}} \frac{1}{f\left(p_{t}^{J / \psi}\right)}=L_{x y}^{J / \psi} \frac{M_{J / \psi}}{p_{t}^{J / \psi}} \frac{1}{f\left(p_{t}^{J / \psi}\right)}
$$


where $L_{x y}^{J / \psi}$ is the displacement of the dimuon vertex from the primary vertex, also defined in the Glossary, $M$ refers to the mass of the meson, and $f\left(p_{t}^{J / \psi}\right)$ is a correction function derived from Monte Carlo, such that $f\left(p_{t}^{J / \psi}\right) \approx \lambda_{J / \psi} / \lambda_{B}$. The effect of using this approximation, will be discussed later in chapter 5 .

Modelling $f\left(p_{t}^{J / \psi}\right)$ The function $f\left(p_{t}^{J / \psi}\right)$ was obtained by using the Monte Carlo methods described in section 4.2. All of the constraints from sections 4.2.4 and 4.2.6 were applied. The resultant $B_{s}^{0}$ and $J / \psi$ transverse momenta distributions were used to calculate $\lambda^{J / \psi}$ on an event by event basis. To do this the mean lifetime of the $B_{s}^{0}$ and $B^{+}$ mesons was used to generate a random value of $\lambda^{B_{s}^{0}}$ and $\lambda^{B^{+}}$. From the value of $\lambda^{B_{s}^{0}}$ the value of $\lambda^{J / \psi}$ was calculated by:

$$
\begin{aligned}
L_{x y} & =\lambda^{B_{s}^{0}} \frac{p_{t}^{B_{s}^{0}}}{M_{B_{s}^{0}}} \\
\lambda^{J / \psi} & =L_{x y} \frac{M_{J / \psi}}{p_{t}^{J / \psi}}
\end{aligned}
$$

The behaviour of $\lambda_{J / \psi} / \lambda_{B}$ with respect to $p_{t}^{J / \psi}$ was histogrammed in figure 4.7. From this distribution a mean and standard deviation were calculated from each vertical row of bins, also illustrated in figure 4.7. Then a polynomial function was used to fit the behaviour of the curve. This polynomial fit was then implemented within the analysis to allow a constraint to be made on $\lambda^{B_{s}^{0}}$. Although the lower error limits of the higher energy $J / \psi$ decays violate the kinematic limit, the effect is small and therefore was ignored.

\begin{tabular}{|c|c|}
\hline \hline Description & Constraint \\
\hline Angle between $\gamma \& J / \psi$ & $<1.2$ radian \\
$E(\gamma)$ & $>0.8 \mathrm{GeV}$ \\
Hadronic/Electromagnetic & $<0.05$ \\
$|m(\gamma \gamma)-m(\eta)|$ & $<120 \mathrm{MeV}$ \\
$p_{t}\left(B_{s}^{0}\right)$ & $>6.0 \mathrm{GeV}$ \\
$c \tau_{\text {approx }}$ & $>100 \mu \mathrm{m}$ \\
\hline \hline
\end{tabular}

Table 4.5: A summary of the constraints made on the $\eta$ candidate and the $B_{s}^{0}$ candidate. 

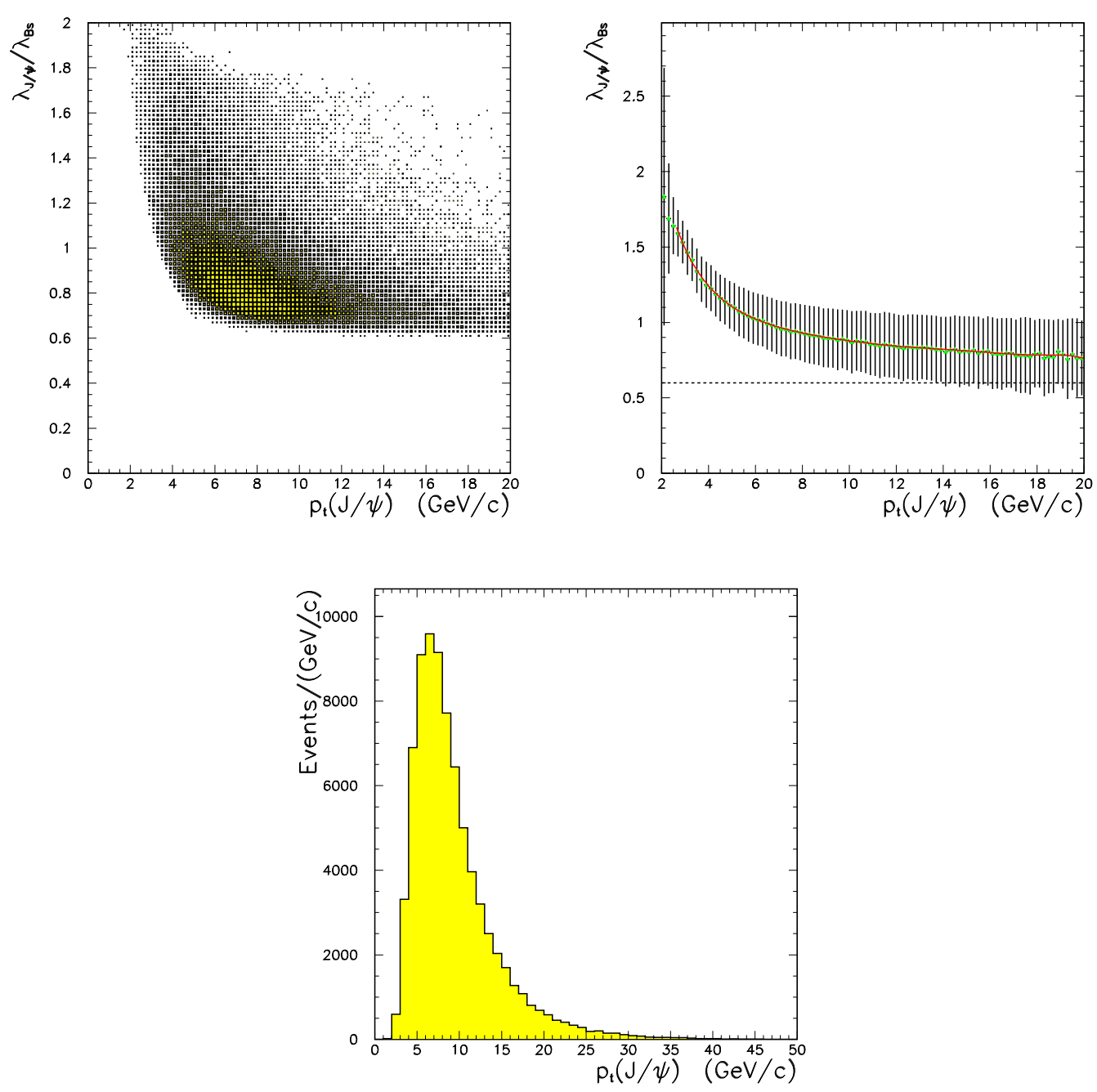

Figure 4.7: Left The distribution of $f\left(p_{t}^{J / \psi}\right)$ values with respect to $p_{t}^{J / \psi}$, for $J / \psi$ of $B_{s}^{0} \rightarrow J / \psi \eta$ decays passing all constraints. Right Mean behaviour of $f\left(p_{t}^{J / \psi}\right)$ with respect to $p_{t}^{J / \psi}$. The error bars shown were calculated from the standard deviation of each vertical bin slice. The dotted line shows the kinematic limit. Bottom The $p_{t}^{J / \psi}$ spectrum of these events.

\subsection{Efficiencies and Acceptances}

\subsubsection{Introduction}

A complete calculation of the branching ratio $\mathcal{B}\left(B_{s}^{0} \rightarrow J / \psi \eta\right)$ required all $K^{ \pm}$and $\eta \rightarrow \gamma \gamma$ acceptance and detector-related efficiencies to be incorporated into the simulation 
described in section 4.2. The detector effects were split into two pieces: geometrical acceptances which were modelled with the simulation directly and the chamber reconstruction efficiencies which were studied using data. This section describes the calculation of the detector efficiency factors.

\subsection{2 $\quad K^{ \pm}$Reconstruction Efficiency}

As previously mentioned in sections 4.2.5 and 4.3.3, the analysis of $B^{ \pm} \rightarrow J / \psi K^{ \pm}$within Monte Carlo and data was based on the analysis described in [39]. The $K^{ \pm}$was chosen from any track, exiting the CTC at a radius greater than $110 \mathrm{~cm}$, and was found to form a vertex with the $J / \psi$ muons. The $110 \mathrm{~cm}$ exit radius was modelled by projecting the $K^{ \pm}$four-vector from the simple Monte Carlo described in section 4.2, into the detector geometry. Then the exit radius constraint was added and the number of Monte Carlo decays passing the constraint calculated. The CTC tracking efficiency was applied by multiplying the final number of events by the efficiency factor illustrated in figure 4.8. Finally the effect of the $\mu^{+}, \mu^{-}, K^{ \pm}$two-dimensional vertex $\chi^{2}$ constraint was included. This is a $\chi^{2}$ with one degree of freedom, the effect of which is discussed in section 4.3.3. Assuming the tracks produced from the $B^{ \pm} \rightarrow J / \psi K^{ \pm}$decay do follow the $\chi^{2}$ relation, then the vertex constraint given in table 4.4 will exclude $0.2 \%$ of the signal. Therefore within the Monte Carlo the $\chi^{2}$ constraint was modelled by excluding $0.2 \%$ of events.

\subsubsection{CES Reconstruction Efficiency}

\subsubsection{Introduction}

The CES reconstruction efficiency is the probability of a photon incident on the CES chamber being reconstructed by the CES. The efficiency was calculated by using conversion electron pairs. Conversion pairs were selected from the electron sample described in [20]. Conversion partners of identified electrons were identified as positrons or electrons by the quality of the conversion vertex. The partner was used in preference to the identified electron because unlike an identified electron track conversion partners are not subject to any calorimetry selection cuts.

The procedure for calculating the CES efficiency for low energy photons has been refined by the CDF collaboration and is described in [13], [12] and [58]. Proper calculation of the efficiency is especially important towards lower energies since the $E_{t}$ 


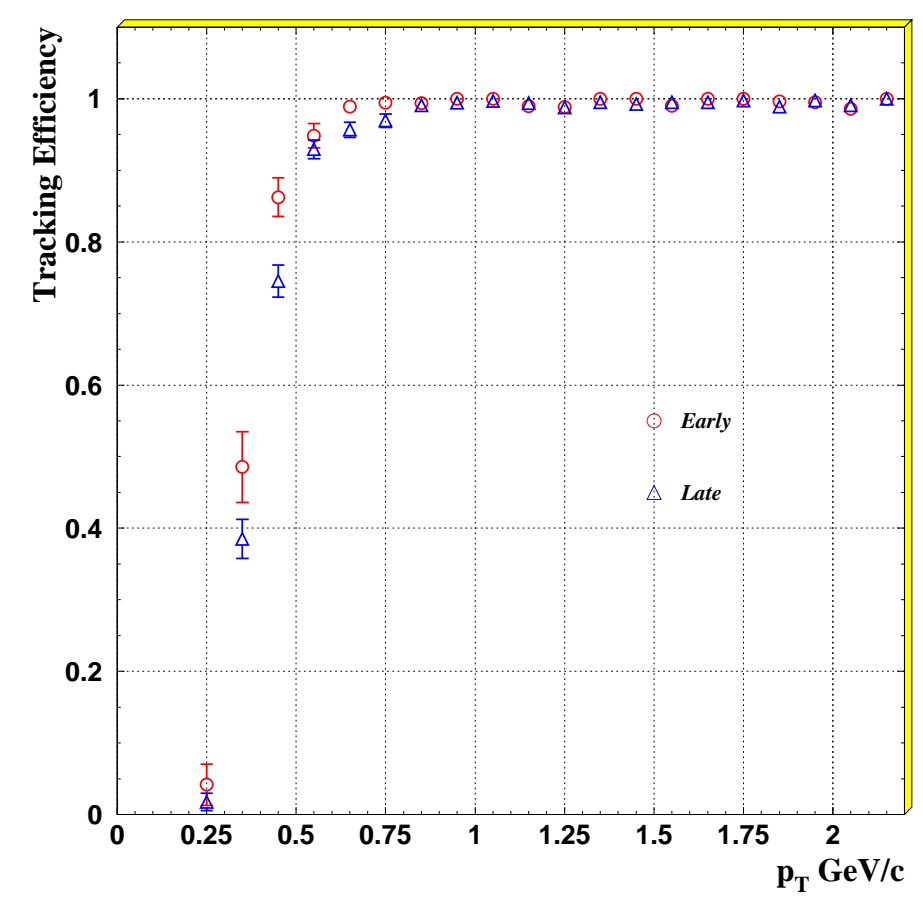

Figure 4.8: CTC Tracking efficiency vs $p_{t}$ for early and late runs, taken from [40]. Combining all runs the single track efficiency for transverse momenta above $0.4 \mathrm{GeV} / \mathrm{c}$ and exit radii greater than $110 \mathrm{~cm}$, was found to be $92.8 \pm 2.0 \%$.

of a photon from a $B_{s}$ daughter $\eta \rightarrow \gamma \gamma$ decay peaks towards low energies, as illustrated in figure C.1. Furthermore unlike previous photon analyses two daughter photons are involved. Therefore the accuracy to which the efficiency curve is known has a stronger influence on the final simulated acceptance.

The rest of this section describes the CES efficiency study used and the improvements made to existing strategies.

\subsubsection{Conversion Pair Selection}

Conversion pairs were selected by scanning the electron sample described in [20] for pairs of tracks which came from $\gamma \rightarrow e^{+} e^{-}$pair production. Each identified electron in the event was compared with every other non-muon track in the event. The muon candidate tracks were excluded by requiring that the track did not have an associated muon stub from reconstruction. Track pairs were assumed to be a conversion pair if: the separation 
at the tangent point was less than $0.2 \mathrm{~cm}$ and the difference $\Delta \cot (\theta)$ was less than 0.3 . The separation at the tangent point is illustrated in figure 4.9 and the difference $\Delta \cot (\theta)$ is:

$$
\Delta \cot (\theta)=\left|\cot \left(\theta_{1}\right)-\cot \left(\theta_{2}\right)\right|
$$

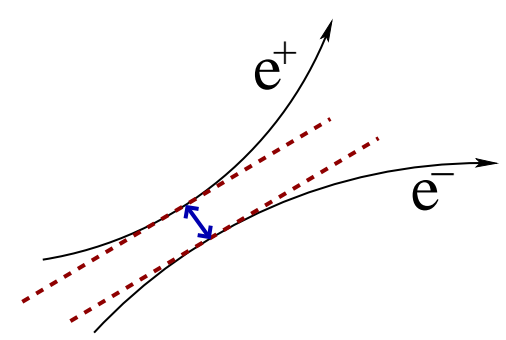

Figure 4.9: Definition of the tangent point between two tracks.

Not all conversion partners are suitable for efficiency studies. Photon pair production occurs when a photon interacts with the material of the beam pipe, inner detector and the inner wall of the CTC. Electrons that are produced by interactions with the beam pipe or the inner detector may well go on to interact with the inner wall of the CTC can, producing a bremsstrahlung photon $e \rightarrow e \gamma$. In this case the ionisation in the CES chamber would be higher than expected from the CTC momentum measurement of the electron. This systematic uncertainty was removed by only selecting conversion partners that were produced by an interaction with the outer part of the inner detector and the inner wall of the CTC can. The radius of conversion for conversion pairs and the selected sample is illustrated in figure 4.10.

\begin{tabular}{|c|c|}
\hline \hline Description & Constraint \\
\hline Separation at tangent & $<0.2 \mathrm{~cm}$ \\
$\Delta \cot (\theta)$ & $<0.3$ \\
Radius of Conversion $(r)$ & $22.0<r<30.0 \mathrm{~cm}$ \\
\hline \hline
\end{tabular}

Table 4.6: Selection criterion for conversion pairs

\subsubsection{Conversion Pair Constraints}

The following requirements made on the conversion pair are similar to those made during the analysis described in [19]. These constraints were made to ensure the validity of the 


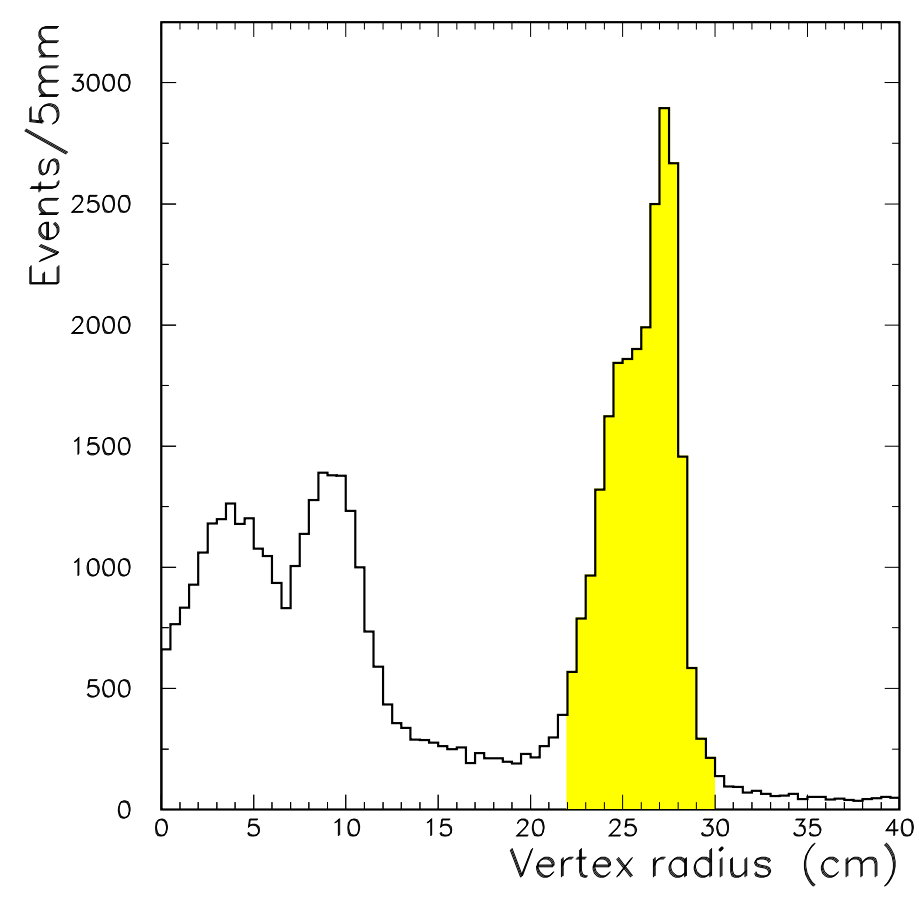

Figure 4.10: A histogram of the radial displacement of $e^{+} e^{-}$conversion vertices from the origin. Conversion electrons produced by interaction with the CTC can and the outermost layer of the inner detector are shown in yellow.

assumed electron status for the identified electron leg, and reduce the possibility of other photon or track objects being present within the local calorimeter volume occupied by the conversion partner. The effect of each constraint that follows is illustrated in a series of plots contained within appendix D.

Identified Electron Cuts The identified electron leg was required to have a $p_{t}>$ $6 \mathrm{GeV} / \mathrm{c}$ and pass electromagnetic shower centroid fitting constraints in the CES chamber. These CES requirements were that both the $\chi^{2}$ 's for the strip channel fit and wire channel fit was required to be less than 15 . These $\chi^{2}$ 's had $(N-1)$ degrees of freedom where $N$ is the number of strips or wire channels involved in the fit. Although seven channels were used for this fit, many events contain some channels equal to zero. Assuming all channels to be non-zero these constraints, when combined, have a probability of $96 \%$. Within the calorimeter tower where the identified electron leg was recorded the ratio of hadronic 
over electromagnetic energy was limited to less than 0.04 excluding hadronic showers. This electromagnetic shower was then required to be properly matched to a helical track. For this to be the case the fitted position of the electromagnetic shower in the CES cluster with respect to strip and wire views had to agree such that $z_{f i t}-z_{\text {hel }}<3 \mathrm{~cm}$ and $x_{f i t}-x_{h e l}<1.5 \mathrm{~cm}$, where the subscripts $f i t$ and hel refer to the fitted and extrapolated positions in the local coordinate system of a calorimeter wedge.

\begin{tabular}{|c|c|}
\hline \hline Description & Constraint \\
\hline$p_{t}$ & $>6 \mathrm{GeV} / \mathrm{c}$ \\
$\chi^{2}$ for CES wire fit & $<15$ \\
$\chi^{2}$ for CES strip fit & $<15$ \\
Had/Em & $<0.04$ \\
$z_{f i t}-z_{h e l}$ & $<3 \mathrm{~cm}$ \\
$x_{f i t}-x_{h e l}$ & $<1.5 \mathrm{~cm}$ \\
\hline \hline
\end{tabular}

Table 4.7: Constraints applied to the identified electron leg of a conversion pair

Plots illustrating the behaviour of all of these cuts can be found in appendix D.

Conversion Partner Electron Cuts Partners of identified electrons passing the quality criterion, were then filtered to remove any other sources of electromagnetic showers. A base line requirement for these conversion partners was made on their transverse momentum, set just above the value at which tracks fail to reach the calorimeter, a transverse momentum cut of $p_{t}>500 \mathrm{MeV} / \mathrm{c}$. The conversion partner was required to be inside CEM tower 0 to 8 . Again the chimney tower was avoided because the CES chamber in that calorimeter module does not extend into the chimney tower. To ensure good energy separation the conversion partner and the identified electron leg were required to be separated by at least one wedge. Minimising the possibility of another electromagnetic shower within the tower, no tracks except the conversion partner were allowed within the wedge. Finally to reduce the possibility of hard photons within the local CEM region, the summed energy recorded within the conversion partner wedge was required to be less than $0.5 \mathrm{GeV}$ after subtraction of the energy from the conversion partner.

Plots illustrating the behaviour of all of these cuts can be found in appendix D. For 


\begin{tabular}{|c|c|}
\hline \hline Description & Constraint \\
\hline$p_{t}$ & $>500 \mathrm{MeV} / \mathrm{c}$ \\
$\left(\sum E M\right)-E M_{\text {partner }}$ & $<0.5 \mathrm{GeV}$ \\
\hline \hline
\end{tabular}

Table 4.8: Cuts applied to the un-biased conversion partner, where $\left(\sum E M\right)$ implies the total electromagnetic energy within the CEM module.

CES clustering to be effective the conversion electron was required to be within two cells of the boundary: previously described in section 4.2.6.

\subsubsection{Calculating the CES Reconstruction Efficiency}

If the conversion pair that passed all of the constraints outlined in section 4.4.3.3, then the unbiased conversion partner was considered to be a photon candidate tower. All such candidate towers in each event were passed to the photon reconstruction code. The ratio of the transverse energy $\left(E_{t}\right)$ spectrum for these conversion partners, before and after requiring CES reconstruction is shown in figure 4.11. The errors for each point were assumed to be binomial. The form of the binomial error [22] is:

$$
\sigma=\sqrt{N p(1-p)}
$$

where $N$ is the number of events within the sample and $p$ is the probability of success in a single observation. For the efficiency histogram figure $4.11, p=n / N$ where $n$ is the number of observed events after the constraint has been made and $N$ is the number of events in the original sample.

In figure 4.11 there is a smooth rising edge corresponding to low energy electrons. Since the two photons produced from a $B_{s}^{0} \rightarrow J / \psi \eta$ decay are both likely to be low energy i.e. within the turn on region of figure 4.11, a closer look at the accuracy of the efficiency calculation was taken.

Sample Differences In terms of their behaviour within the CEM, there are three main differences between photons produced from $B_{s}^{0} \rightarrow J / \psi \eta$ decays and conversion electrons: the production vertex, charge i.e. helical nature, and the longitudinal shower profile.

Photons from $B_{s}^{0} \rightarrow J / \psi \eta$ decays are accepted with a distribution indicated in figure A.1. Conversion electrons however have an equal probability of being produced 


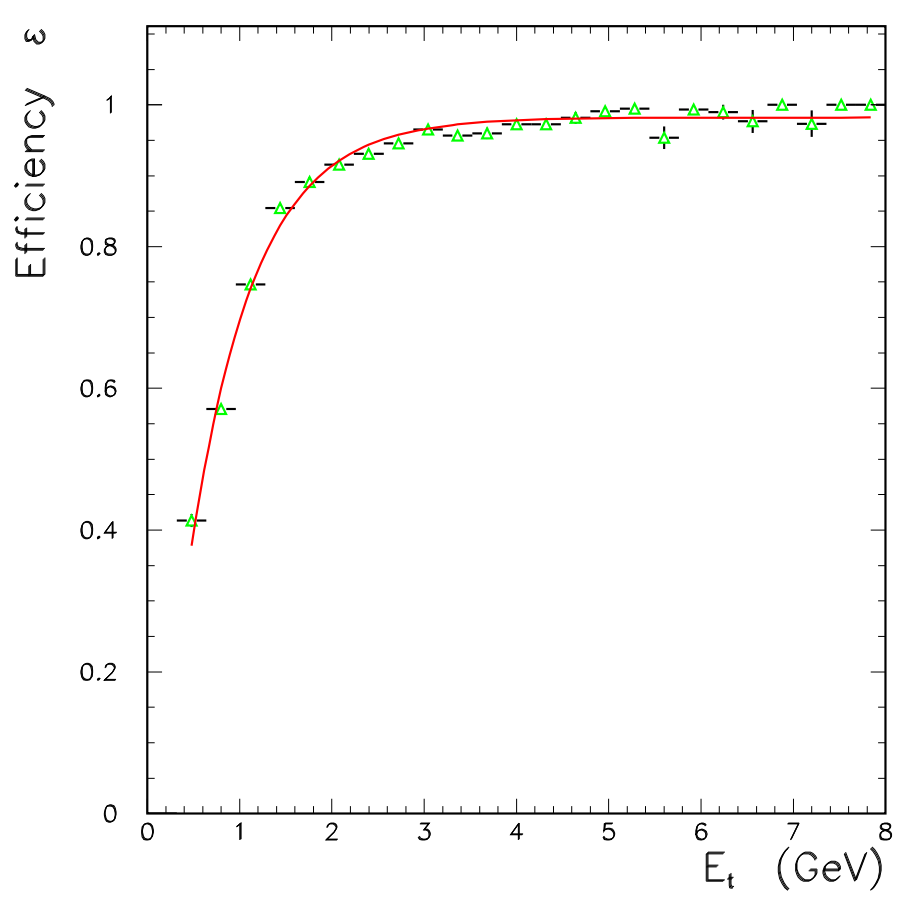

Figure 4.11: The efficiency of finding a CES cluster within a tower into which a conversion electron partner track projected.

anywhere along the surface of the inner detector or CTC inner can, as illustrated in figure 4.12 .

Relative to the $J / \psi$ vertex distribution the conversion vertex distribution is heavily weighted towards the edges of the detector. Since the CEM is a pointing calorimeter there is an increased chance that the conversion electrons, having entered a CEM tower will leave the tower before reaching the CES. Beyond this the conversion electrons on average will see less calorimeter material if perpendicular to the surface of the 8th tower for example. The first concern was removed by requiring that the projected position at the CES be inside the tower which was first entered.

As previously mentioned the angle in the $r-z$ plane is not the same on average for conversion electrons and photons. This effects the amount of material which is traversed but is compounded by the helical nature of the electrons. Once a low energy electron track is produced it bends in the 1.4 Tesla axial field so that it enters a CEM tower at steeper angle compared to a straight track. This effect is magnified by the radial displacement of 


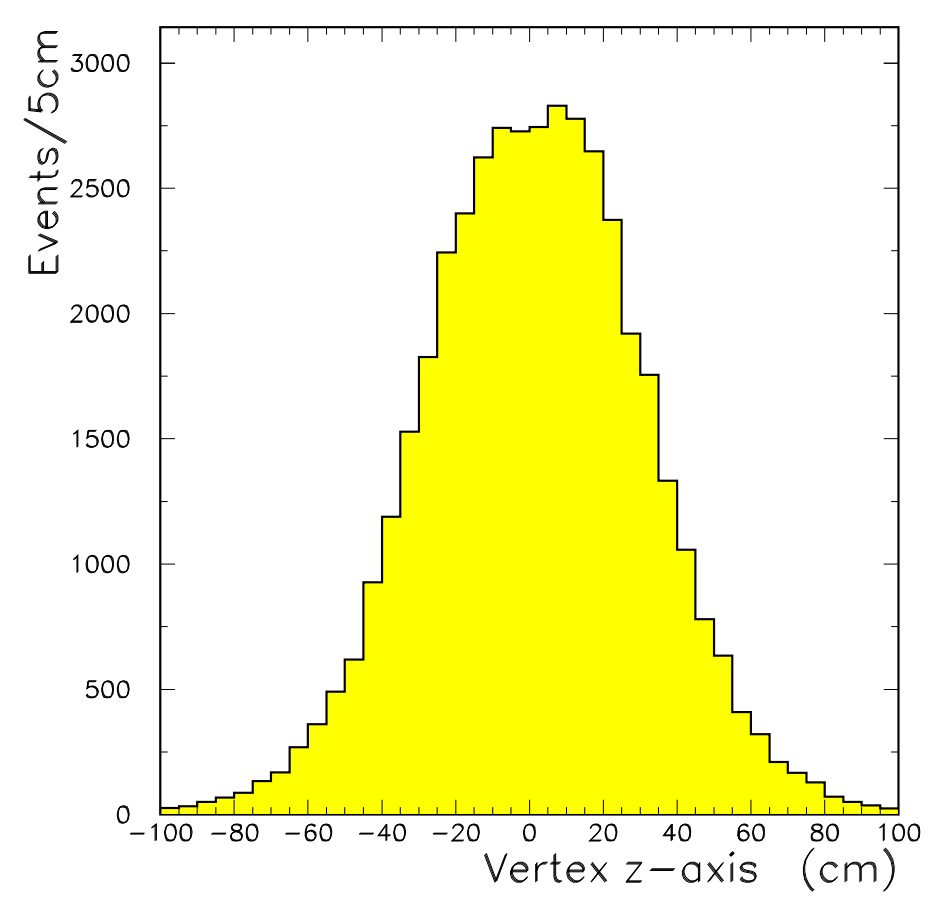

Figure 4.12: The distribution of $e^{+} e^{-}$conversion vertices along the beam axis where each conversion pair passed all of the constraints described in section 4.4.3.3

the conversion vertex, shown in figures 4.10.

If all conversion electron pairs travelled in straight lines from the origin to a CEM tower they would traverse the number of radiation lengths given in table 2.2. This however does not happen. A complete picture of the divergence from this model was produced by calculating the CEM path length for each electron flight and hence the number of radiation lengths seen before the CES, illustrated in figure 4.13. From these results it is clear that a CES reconstruction efficiency plot should be made from predicted energy deposited in the CES and not $E_{t}$.

Corrections All of the conversion electron sample dependencies together with those of photons produced from $B_{s}^{0} \rightarrow J / \psi \eta$ were removed by using the predicted energy deposit instead of $E_{t}$. This means that the conversion electron predicted energy deposit was histogrammed before and after requiring photon reconstruction. The ratio of these two plots was then used to decide if a photon generated within the simulation should be 


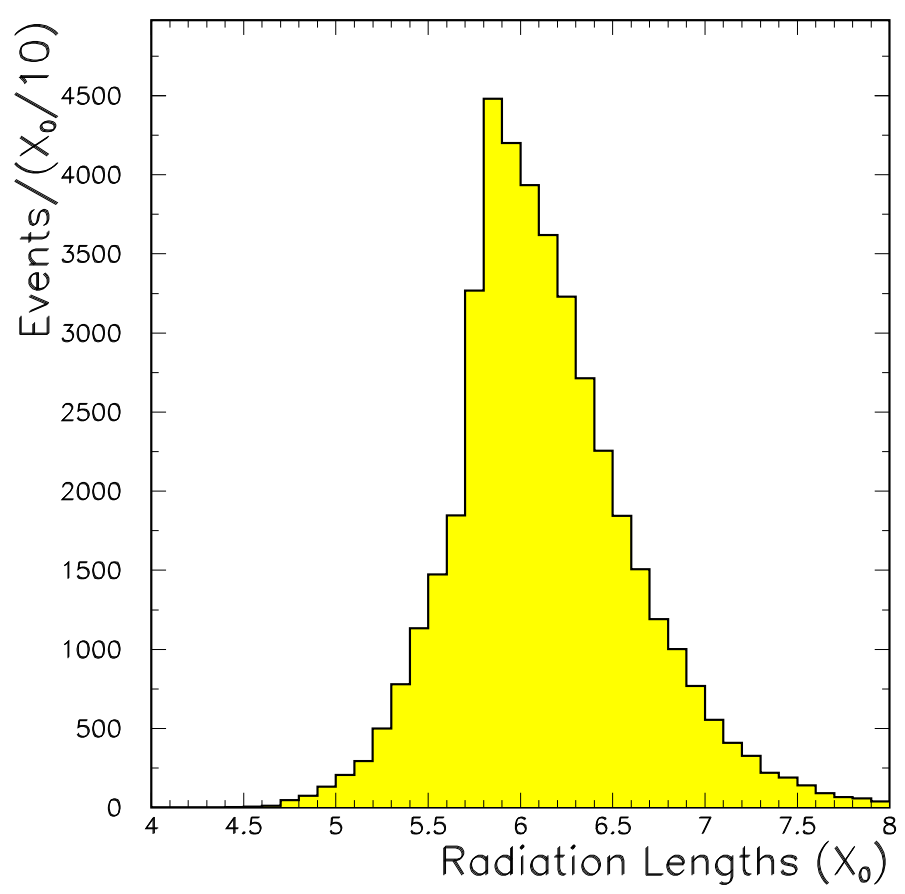

Figure 4.13: The distribution of radiation lengths seen by a conversion partner electron crossing the solenoid and electromagnetic calorimeter material in front of the CES chamber

reconstructed based on its predicted CES deposit. This predicted CES energy deposit was calculated on an event by event basis using equation 4.11 . This equation was taken from [35] and is a parameterisation of the more well known form of the longitudinal shower progression equation given in [33]. The form given in equation 4.11 was used to allow simple incorporation of the electron test beam fits.

$$
\frac{d E}{d x}=\frac{E_{0} A b(b x)^{b x_{M A X}} e^{-b x}}{\Gamma\left(b x_{M A X}+1\right)}
$$

where

$$
\begin{gathered}
x_{M A X}=\ln \left(\frac{E_{0}}{E_{c}}\right)+C \\
b=p_{1}+p_{2} \ln \left(E_{0} / E_{c}\right)+p_{3}\left[\ln \left(E_{0} / E_{c}\right)\right]^{2}
\end{gathered}
$$

$E_{0}$ is the initial energy of the electron, positron, or photon, $x$ is the number of radiation lengths travelled, $x_{M A X}$ is the position of the shower maximum, $d E / d x$ is the energy 
dissipated per unit radiation length, $E_{c}$ is the critical energy taken as $9.59 \mathrm{MeV}$, and $A$, $p_{1}, p_{2}, p_{3}$ are fit parameters. The values for these fit parameters are given in table 4.9 and were taken from fits to the 1990 electron test beam data [35]. The value of $A$ for $E_{0}<5.0 \mathrm{GeV}$ was chosen to ensure continuity between the two curves. Errors on the value of $A$ for $E_{0}<5.0 \mathrm{GeV}$ were calculated by fixing the continuity between the two curves at the lower and upper limits of the $E_{0}>5.0 \mathrm{GeV}$ fit parameters. The value of the constant $C$, which is quoted as part of $x_{M A X}$, depends on the type of the incident particle. The value of $C_{e}$, (the constant value for an incident electron), was taken as -0.5 from the testbeam fits whereas the value of $C_{\gamma}$, (the constant value for an incident photon), was taken as expected from other fits [35], [27]. The uncertainty connected with this constant is discussed within chapter 5 .

\begin{tabular}{|c|c|c|}
\hline \hline Fit parameter & Value $\left(E_{0}<5.0 \mathrm{GeV}\right)$ & Value $\left(0.1<E_{0}<5.0 \mathrm{GeV}\right)$ \\
\hline$A$ & $3.86 \pm 0.02$ & $4.04_{-0.01}^{+0.045}$ \\
$p_{1}$ & $-0.796 \pm 0.004$ & 0.4 \\
$p_{2}$ & $0.3193 \pm 0.0005$ & -0.063 \\
$p_{3}$ & $-0.01812 \pm 0.00007$ & 0.012 \\
\hline \hline
\end{tabular}

Table 4.9: A table of longitudinal shower fit parameters from [35].

For every event the conversion partner was projected into the calorimeter, its path length was calculated and therefore according to the tower entered the corresponding number of radiation lengths. The $p_{t}$ measured in the CTC was used to calculate the value of $E_{0}$ and hence $a$. Using these values the value of $d E / d x$ was calculated at the CES depth. By histogramming $d E / d x$ before and after the photon reconstruction requirement figure 4.15 was produced.

Adding the CES Efficiency to the Monte Carlo Once the CES reconstruction efficiency had been calculated with respect to the predicted CES energy deposit $d E / d x$, the fit shown in figure 4.15 was used within the simulation described in section 4.2. The efficiency curve was fitted with an exponential. The fit values for the exponential are given in table 4.10 .

On an event-by-event basis the path length from the solenoid to the CES was calculated 


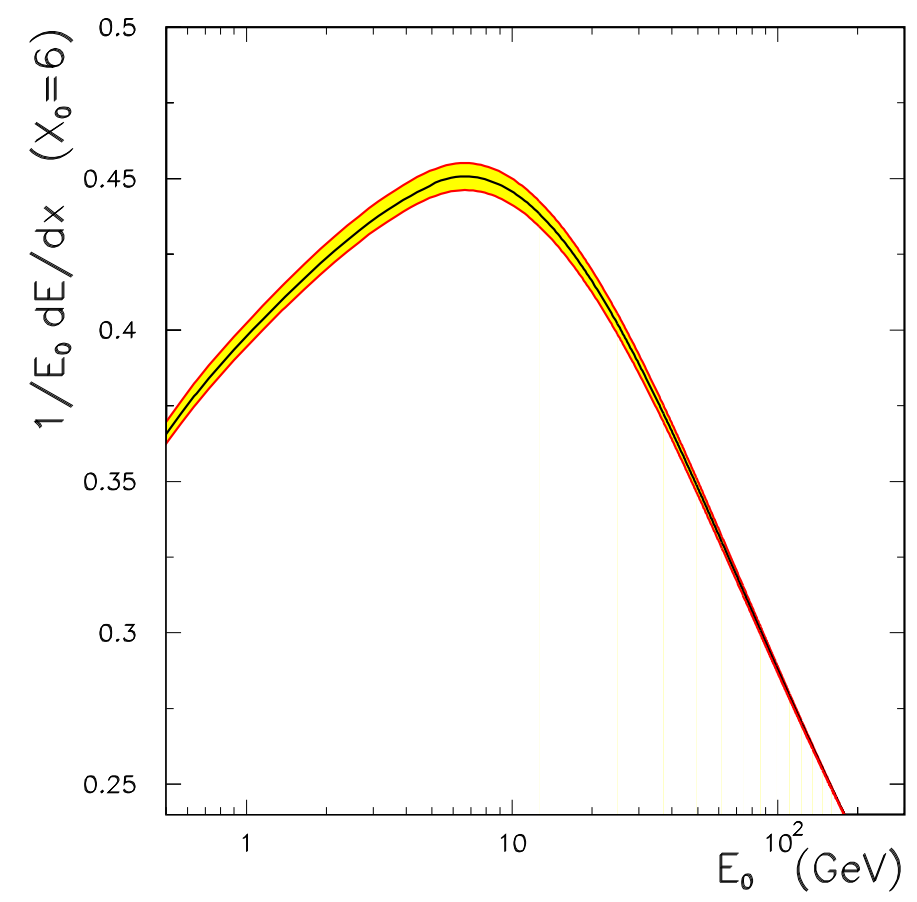

Figure 4.14: Longitudinal shower progression using fit parameters given in table 4.9 and assuming the CES to be at a constant depth of six radiation lengths.

\begin{tabular}{|c|c|}
\hline \hline Fit parameter & Value \\
\hline$p_{1}$ & $2.45 \pm 0.14$ \\
$p_{2}$ & $0.027 \pm 0.025$ \\
$p_{3}$ & $1.00 \pm 0.015$ \\
\hline \hline
\end{tabular}

Table 4.10: CES efficiency curve fit parameters, where $y=p_{3}\left(1-\exp \left(p_{2}-p_{1} x\right)\right)$

for each generated photon. According to the specific tower this path-length was converted into a number of radiation lengths. Then using the energy of the generated particle and the calculated depth to the CES the probability of reconstruction was calculated. Then using a random number generator each photon was/was not selected as passing the reconstruction.

Photons and electrons have different longitudinal shower profiles [33]. The value of $d E / d x$ was calculated for each electron using $C=C_{e}$ were $C_{e}=-0.5$. From [35], the value of $C_{\gamma}$ was chosen such that $C_{\gamma}-C_{e}=0.6$. 


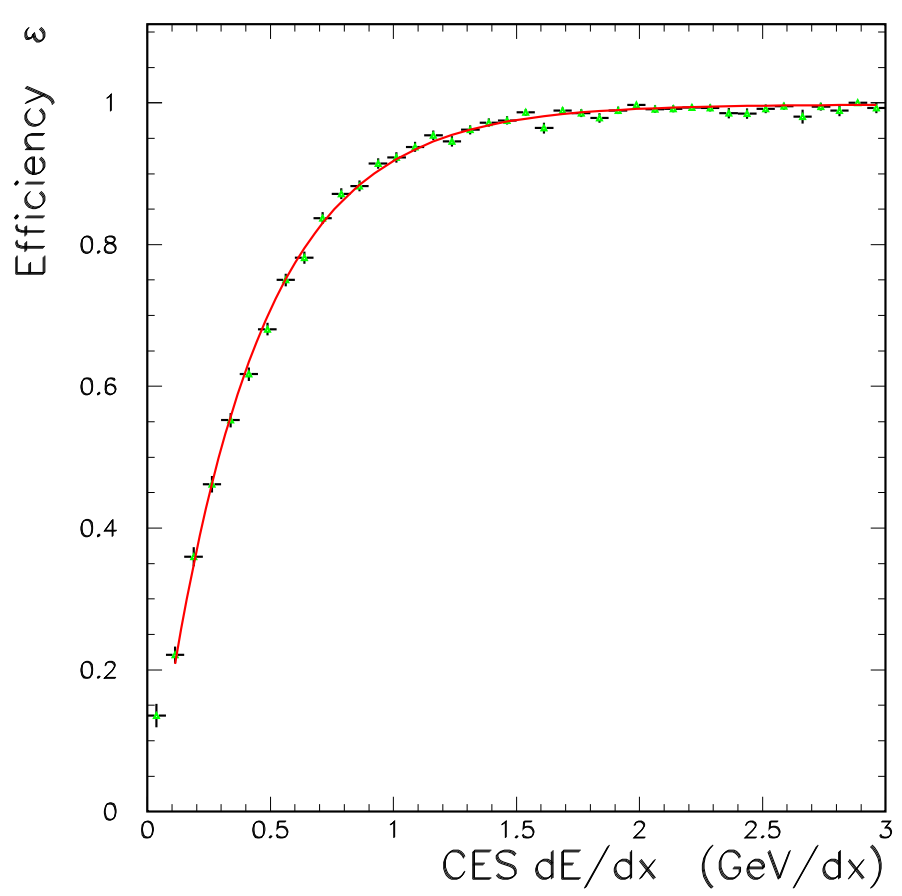

Figure 4.15: The reconstruction efficiency for an electromagnetic shower within the CES with respect to the predicted energy measurement within the CES. The error bars shown were calculated from the binomial error given in equation 4.10 .

\subsection{Detailed Simulation}

When a tower contains two photon like objects, or rather two clusters in the CES, there is a chance that when searching for photon daughters of an $\eta$ meson, that the wrong CES cluster could be chosen. Beyond this problem the CES clustering could fail if there is an ambiguity, or if one of the cluster views is superimposed. To tackle both of these problems a detailed detector simulation, containing correctly modelled backgrounds was used. Since there are currently no Monte Carlo programs that completely model CDF events the simulation was constructed from existing data together with Monte Carlo particles. The decay $B^{ \pm} \rightarrow J / \psi K^{ \pm}$has approximately the same background as $B_{s}^{0} \rightarrow J / \psi \eta$. Isolating $B^{ \pm} \rightarrow J / \psi K^{ \pm}$events the $B^{ \pm}$energy was corrected to form a $B_{s}^{0}$ invariant mass. Then a $\eta$ meson was added in place of the $K^{ \pm}$, from which two daughter photons were generated. The photons were projected into the calorimetry and the output data was re-analysed, 
with the $B_{s}^{0} \rightarrow J / \psi \eta$ data analysis constraints.

\subsection{1 $\quad B^{ \pm} \rightarrow J / \psi K^{ \pm}$event selection}

$B^{ \pm} \rightarrow J / \psi K^{ \pm}$events were first selected with the constraints described in section 4.3.3. As much background as possible was removed by lowering the two-dimensional $\mu^{+} \mu^{-}$, $K^{ \pm}$, vertex $\chi^{2}$ constraint to 3.0 , corresponding to a probability of $92 \%$. Then the events within $60 \mathrm{MeV} / \mathrm{c}^{2}$ of the world average $B^{ \pm}$mass were saved to an output file. The event selection is illustrated in figure 4.16 .

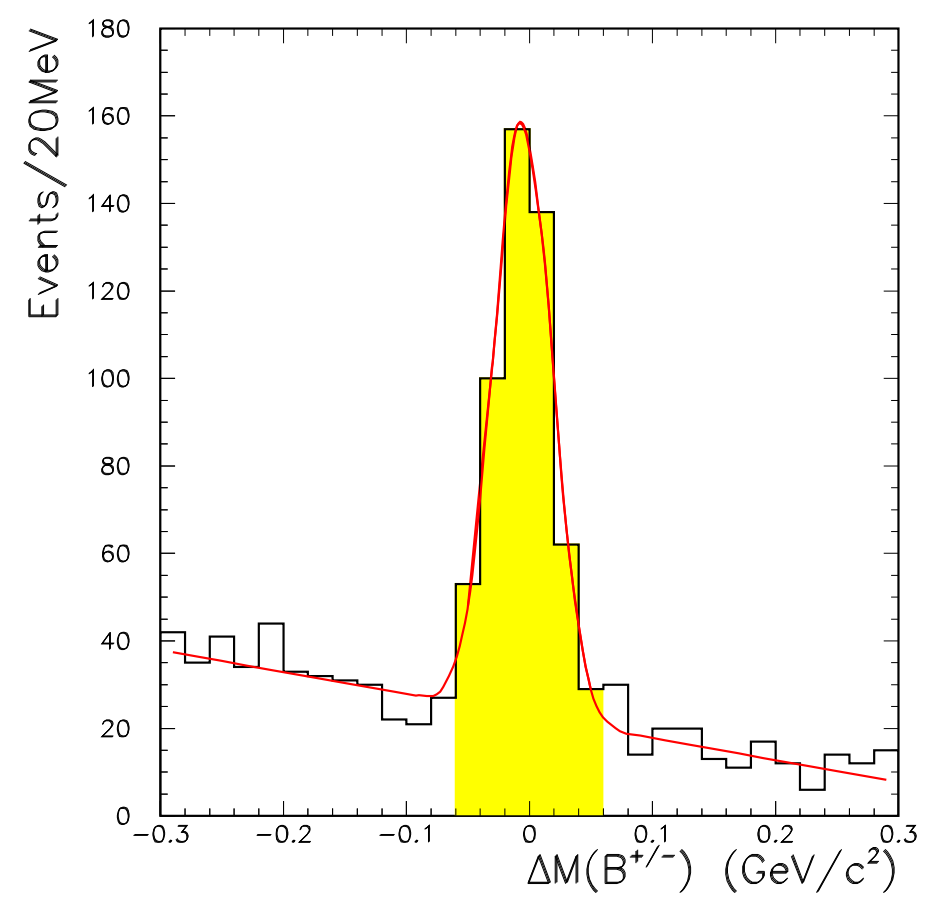

Figure 4.16: Selected $B^{ \pm} \rightarrow J / \psi K^{ \pm}$events, where: $\Delta M\left(B^{ \pm}\right)=m_{B^{ \pm}}^{P G}-m_{B^{ \pm}}^{\text {meas }}$

\subsubsection{Modelling photons in the calorimeters}

Once a high quality $B^{ \pm}$sample had been obtained a better model of photons within the CEM and its CES was sought. This model was required to correctly describe the detector response of low energy photons (below $5 \mathrm{GeV}$ ). In previous simulation work described in section 4.2.6 photon detector simulation only included energy smearing by the CEM energy resolution. In order for photons to be correctly modelled within the 
CEM and its CES chamber, photon objects were needed with their associated CES charge per channel. The CDF collaboration has previously modelled electromagnetic showers within the CEM with QFL[36]. QFL models electromagnetic shower profiles within the CES chamber by selecting electron testbeam shower profiles and then adding them to the detector information. The lowest energy showers available within QFL are $5 \mathrm{GeV}$, more energetic than most photons produced from $B_{s}^{0} \rightarrow J / \psi \eta$ decays. Following the QFL principle but needing lower energy electromagnetic showers, two sources of electron shower profiles were considered: $\pi^{0}$ photons coming from a dimuon vertex and conversion partner electrons. $\pi^{0}$ photons were not used, since most of the candidate events were such that the electromagnetic showers in the CES clusters were too close to separate. Therefore $\pi^{0}$ photons were abandoned and conversion partners were chosen as the next best solution.

Selecting low energy electromagnetic showers Conversion electrons were selected with the constraints described in section 4.4.3. Then from this sample of conversion partners, unbiased by electron cuts, further cuts were made to ensure high quality CES shower profiles. Each conversion partner electron was required to point at a CEM tower, behind which there was only one reconstructed CES cluster. To prevent some loss of charge the CES cluster was only kept if its centre was three wire and strip cells from the half chamber boundaries. Before selected showers could be used within a detector simulation, all detector non-homogeneities had to be removed. These non-homogeneities are described in [56]. To remove the wire pulse height effects gain curves documented in [56] were applied on a channel-by-channel basis. These curves are only valid within the local coordinate range $|x|<20 \mathrm{~cm}$. Therefore the CES centroid was required to be in this range. To ensure no electromagnetic energy leaked into the conversion partner's CEM tower, the sum energy recorded by the neighbouring eight CEM towers was constrained to be $<0.5 \mathrm{GeV}$. All CES profiles that fulfilled these selection criterion were then saved to disk along with the predicted CES energy deposit, the CEM energy, and the fitted position of the shower centroid. Just before saving these data to disk the wire pulse height was renormalised and the strip to wire gain maps were applied to remove localised detectorrelated effects. Data that were written to disk were then scanned for multiple instances of showers within one CES chamber view. These events were occasionally present where the recorded cluster centroid was close to the CEM tower boundary of a neighbouring tower 
containing a CES cluster. Under these conditions, when writing $3+1+3$ channels to disk, channels from the neighbouring tower would have also been included. Once events containing multiple CES clusters were removed the data were sorted into order according to their corresponding predicted CES pulse height.

\begin{tabular}{|c|c|}
\hline \hline Description & Constraint \\
\hline CES $|x|$ & $<20 \mathrm{~cm}$ \\
8 CEM tower sum & $<0.5 \mathrm{GeV}$ \\
\hline \hline
\end{tabular}

Table 4.11: Constraints applied to the conversion partner to isolate a sample of low energy CES shower profiles.

\subsubsection{Generating $B_{s}^{0} \rightarrow J / \psi \eta$ events}

Events passing selection criterion described in section 4.5.1, were re-read. The energy of the $B^{ \pm}$was corrected so that the invariant mass was that of a $B_{s}^{0}$ meson. The four-vector of the real $J / \psi$ particle was boosted into the rest frame of the $B_{s}^{0}$. Then in the rest frame of the $B_{s}^{0}$ meson the $\eta$ meson was found by simple momentum conservation. In the rest frame of the $\eta$ particle the photons were produced back to back, boosted back into the laboratory frame and smeared according to the CEM energy resolution. The kinematic limit of both photons produced transverse to the direction of the $\eta$ meson was used to test if the event would pass the $E_{t}$ cut before the photon production axis was generated. Events that passed the kinematic limit were regenerated with different decay photon decay axes until they were excepted by the $E_{t}$ cut. (Since the number of $B^{ \pm}$events recorded was low each event was used if at all possible to keep the final statistics as high as possible.)

Generated photons were then projected into the detector, where they were required to satisfy the geometrical constraints previously described in section 4.2.6. Photons failing the geometrical constraints were regenerated until they were accepted or it was obvious that the kinematics of the event would not allow acceptance. The energy of each accepted photon together with its trajectory was used to calculate the number of radiation lengths to the CES and hence the predicted CES energy deposit. Knowing the predicted CES energy deposit, the CES channel values were re-weighted for local detector effects and then added to the contents of the corresponding chamber. 


\subsubsection{CES purity and rate of ambiguity}

The CES reconstruction efficiency as a function of the predicted energy deposit was calculated as described within section 4.4.3. This is not the only factor involved in the overall CES reconstruction efficiency.

Ambiguity If the both photons from the decay of an $\eta$ fall within the same half of the CES and their energy is reasonably close, (i.e. within the level of the strip or wire noise), there is a chance of an ambiguity. In this example, assuming no other energy in the CES there would be two CES wire clusters and two CES strip clusters. Resulting in the problem illustrated in figure 4.17.

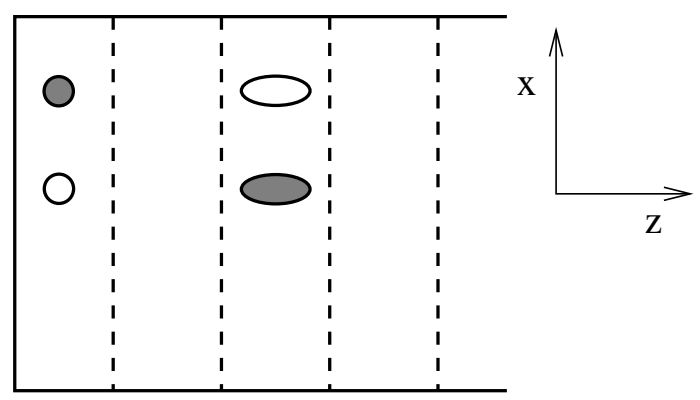

Figure 4.17: A figure illustrating a possible CES ambiguity scenario. The real photon pair shower cross-section at the CES depth is shown in grey.

With the procedure described in section 4.5.3 events were generated to test the rate of ambiguities. These events were constrained to have both photons within the same half of the CES chamber. Then events were looked for where either the reconstructed $x$ or $z$ within the chamber was swapped with the other cluster. The results were plotted by constructing the histograms shown in figure 4.18. The off-diagonal or 'ghost' position is simply the position represented by swapping one of the two coordinates for that of the other cluster in the CES chamber as indicated in figure 4.17. 

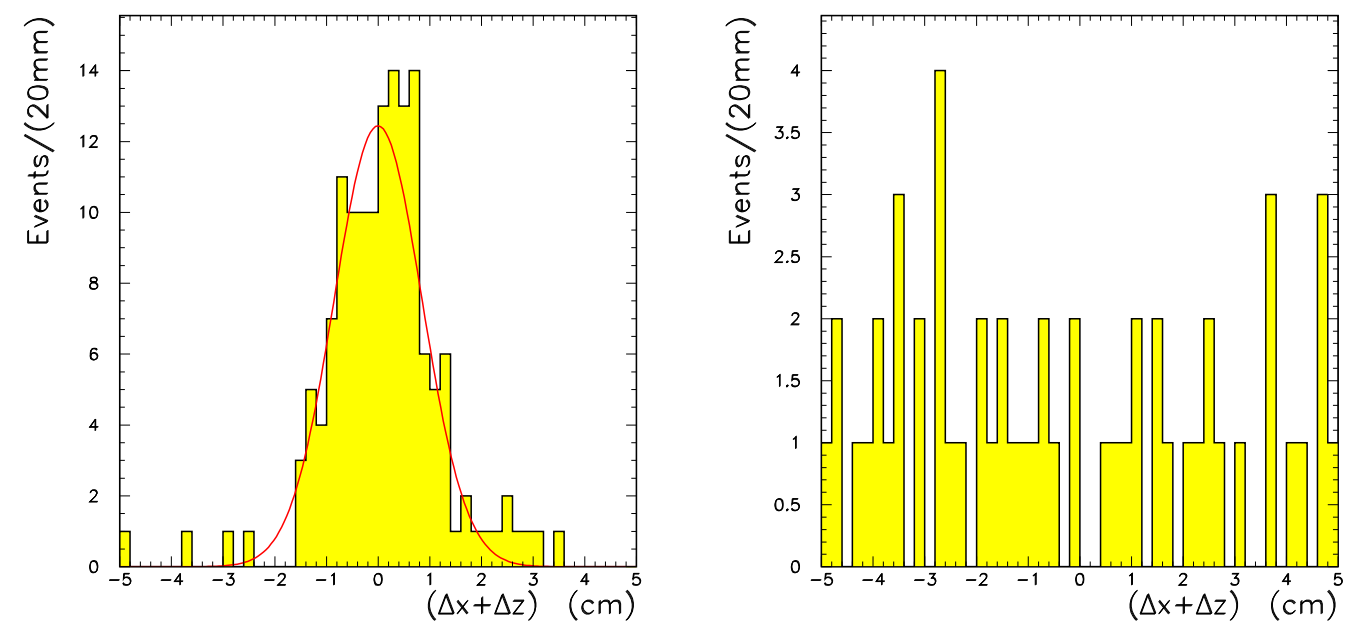

Figure 4.18: Left A histogram of the fitted shower centroid position from CES clustering minus the original position of the particle. Right A histogram of the fitted shower centroid position from CES clustering minus the off diagonal 'ghost' cluster position. 


\section{Chapter 5}

\section{Statistical and Systematic Errors}

\subsection{Introduction}

A measurement is only as good as its error limits. Within this chapter all sources of systematic uncertainties encountered during the analysis are discussed. The chapter is organised into errors from quantities outside the analysis, errors within the simulation methods employed and errors from the data analysis.

The uncertainties connected with measuring a branching ratio for the decay $B_{s}^{0} \rightarrow$ $J / \psi \eta$ can be grouped together into errors internal to this analysis and external i.e. those for which results from other analyses were used. Errors contained in the ratio equation 6.1, are systematic, external to this analysis and are discussed in section 5.2.

Within this analysis errors enter into two final numbers, discussed in chapter 6 , the predicted number of $B_{s}^{0} \rightarrow J / \psi \eta$ events and the $\mathcal{B}\left(B_{s}^{0} \rightarrow J / \psi \eta\right)$ limit. The only difference between the two is that one contains the theoretical uncertainty of a $\mathcal{B}\left(B_{s}^{0} \rightarrow J / \psi \eta\right)$ estimate and the other contains statistical errors from a number of events.

\subsection{External Errors}

Before discussing any of the systematic errors connected with the data analysis ending in the predicted number of events or the confidence limit, the errors from outside the analysis but incorporated into the ratio in equation 6.1, are tabulated in table 5.1.

From table 5.1 it is clear that the uncertainty on the quantity $f_{s} / f_{u}$ is the dominant factor. 


\begin{tabular}{|c|c|}
\hline \hline Quantity & Value \\
\hline$f_{s} / f_{u}$ & $0.215 \pm 0.071[55]$ \\
$\mathcal{B}\left(B^{+} \rightarrow J / \psi K^{+}\right)$ & $(1.0 \pm 0.1) \times 10^{-3}[33]$ \\
$\mathcal{B}(\eta \rightarrow \gamma \gamma)$ & $0.3933 \pm 0.0025[33]$ \\
\hline$\frac{f_{u}}{f_{s}} \frac{\mathcal{B}\left(B^{+} \rightarrow J / \psi K^{+}\right)}{\mathcal{B}(\eta \rightarrow \gamma \gamma)}$ & $0.012 \pm 0.004$ \\
\hline \hline
\end{tabular}

Table 5.1: Errors from parameters not measured during this analysis, but incorporated to form the confidence limit

\subsection{Monte Carlo Ratio}

\subsubsection{Introduction}

The Monte Carlo ratio is given in equation 4.4 as the fraction:

$$
\frac{M C(J / \psi \eta)}{M C\left(J / \psi K^{+}\right)}
$$

This ratio, as discussed in chapter 4, incorporates theoretical production models, trigger acceptances, detector acceptances, and detector tracking efficiencies. With the exception of the tracking efficiency within the CTC all detector related effects are dependent on momentum or energy of the daughter particles. Therefore each one was implemented on a generated event by event basis. Each systematic error was explored by regenerating both Monte Carlo samples and recalculating the ratio for the systematic under examination.

\subsubsection{B Meson Production}

B mesons were produced within the simulation using the methods described in section 4.2.2. The theoretical uncertainty is commonly expressed by variation of the scale $\mu$ and b quark mass $m_{b}$. Following the prescription outlined in [18], for which input values are summarised in table 5.2, both Monte Carlo samples were regenerated for the three limits. Taking trigger acceptances, detector acceptance and efficiency effects into account the Monte Carlo ratio was calculated, as described in chapter 4. The upper and lower error systematic error limits are therefore given by difference between the central and outer limits. These values are summarised in table 5.3. 


\begin{tabular}{|c|c|c|c|}
\hline \hline & Lower & Upper & Central \\
\hline$m_{b}$ & $4.5 \mathrm{GeV} / \mathrm{c}^{2}$ & $5.0 \mathrm{GeV} / \mathrm{c}^{2}$ & $4.75 \mathrm{GeV} / \mathrm{c}^{2}$ \\
$\mu$ & $\mu_{0} / 2$ & $2 \mu_{0}$ & $\mu_{0}$ \\
$\epsilon_{Q}$ & 0.004 & 0.008 & 0.006 \\
\hline \hline
\end{tabular}

Table 5.2: Input value ranges used during B meson production: where $m_{b}$ is the b quark mass, $\mu$ is the renormalisation and factorisation scale and $\epsilon_{Q}$ is the tuning parameter for Peterson fragmentation.

\begin{tabular}{|c|c|c|}
\hline \hline$M C(J / \psi \eta) / M C\left(J / \psi K^{+}\right)$ & Lower Variation & Upper Variation \\
\hline 0.2178 & $0.9 \%$ & $-0.9 \%$ \\
\hline \hline
\end{tabular}

Table 5.3: Systematic errors within B meson production

\subsubsection{Modelling $J / \psi \rightarrow \mu^{+} \mu^{-}$}

\subsubsection{Primary Vertex Generation}

Each event generated by the simulation was given a primary vertex position based on the distribution observed from $J / \psi \rightarrow \mu^{+} \mu^{-}$data. The distribution was formed by histogramming the primary vertex for muon pairs. Each muon pair satisfied $\chi^{2}$ matching constraints between track and stub and was attached to three or more SVX hits. Further description is given in section A.1.

The distribution is determined by tracking efficiencies within the SVX is not a regular shape, and an attempt to fit the distribution with a curve failed. Therefore the distribution was used directly to generate primary vertices. The error connected with this primary vertex distribution was simply calculated from $1 / \sqrt{N}$ where $N$ is the number of entries in each bin. To calculate the effect of limited accuracy within the primary vertex position the two Monte Carlo samples were generated with upper and lower values for all bins. The results are given in table 5.4.

\begin{tabular}{|c|c|c|}
\hline \hline$M C(J / \psi \eta) / M C\left(J / \psi K^{+}\right)$ & Lower Variation & Upper Variation \\
\hline 0.2178 & $0.006 \%$ & $-0.01 \%$ \\
\hline \hline
\end{tabular}

Table 5.4: Systematic errors within primary vertex generation. 


\subsubsection{2 $J / \psi \rightarrow \mu^{+} \mu^{-}$Trigger}

After each event was generated the muon daughters from the $J / \psi$ decay were required to pass the level 1 and 2 triggers. The trigger acceptance curves are illustrated in figures A.6 and A.7. As mentioned in sections A.3 and A.4, the error limits of these curves were obtained by assuming all the fit parameters were completely correlated. The error limits are a result of varying the all of the fit parameters by one sigma of their error. The fitting errors for the two trigger curves were assumed to be uncorrelated so the Monte Carlo ratio was generated for the upper and lower limits for both trigger curves. The results are given in tables 5.5 and 5.6.

\begin{tabular}{|c|c|c|}
\hline \hline$M C(J / \psi \eta) / M C\left(J / \psi K^{+}\right)$ & Lower Variation & Upper Variation \\
\hline 0.2178 & $0.12 \%$ & $-0.028 \%$ \\
\hline \hline
\end{tabular}

Table 5.5: Systematic errors within the level $1 J / \psi \rightarrow \mu^{+} \mu^{-}$trigger acceptance curve fits.

\begin{tabular}{|c|c|c|}
\hline \hline$M C(J / \psi \eta) / M C\left(J / \psi K^{+}\right)$ & Lower Variation & Upper Variation \\
\hline 0.2178 & $-0.068 \%$ & $-0.028 \%$ \\
\hline \hline
\end{tabular}

Table 5.6: Systematic errors within the level $2 J / \psi \rightarrow \mu^{+} \mu^{-}$trigger acceptance curve fits.

\subsection{4 $K^{ \pm}$Reconstruction Efficiency}

The $K^{ \pm}$reconstruction efficiency is unlike all other corrections applied to the Monte Carlo ratio. It is not dependent on $p_{t}$ for tracks with $p_{t}$ above $1.25 \mathrm{GeV} / \mathrm{c}$. This is illustrated in figure 4.8. From [39] the systematic uncertainty for the reconstruction efficiency is $\pm 3.1 \%$.

In addition to the kaon reconstruction efficiency uncertainty there is a chance of decay in flight. Using a simulation it is found in [8], that $8 \%$ of the kaons decay in flight though $50 \%$ were successfully reconstructed. Therefore this systematic error is taken as $\pm 4 \%$. 


\subsubsection{CES Reconstruction Efficiency}

During the simulation each generated photon pair was required to be inside the calorimeter, within the CES, and be reconstructed by the CES. The reconstruction efficiency was applied as previously described in section 4.4.3 and includes two uses of the longitudinal shower progression and a fit to the conversion electron data. Taking a conservative approach, the uncertainties from the longitudinal fit and the final fit to the conversion electron $\mathrm{dE} / \mathrm{dx}$ curve were assumed to be fully correlated. Therefore under this assumption, three curves were fed into the simulation: the upper, lower and central $\mathrm{dE} / \mathrm{dx}$ curves. Then within the simulation the photon $\mathrm{dE} / \mathrm{dx}$ was calculated with the upper, lower and central fit parameters for longitudinal shower progression. Further than this the uncertainty on the value of $C_{\gamma}-C_{e}$, (where $C$ is defined in equation 4.11), was added: again using the corresponding upper, lower and central values. The systematic error limits for $C_{\gamma}-C_{e}$ were taken as the difference between 0.6 given in [35] and 0.5 given in [27], i.e. $0.6 \pm 0.1$. A summary of the systematic error attached to the usage of the CES reconstruction efficiency within the simulation is given in table 5.7 .

\begin{tabular}{|c|c|c|}
\hline \hline$M C(J / \psi \eta) / M C\left(J / \psi K^{+}\right)$ & Lower Variation & Upper Variation \\
\hline 0.2178 & $-1.73 \%$ & $0.59 \%$ \\
\hline \hline
\end{tabular}

Table 5.7: Systematic errors from the longitudinal shower fit parameters and CES efficiency fit to conversion electron data.

\subsection{Data Analysis}

\subsection{1 $\quad B^{ \pm} \rightarrow J / \psi K^{ \pm}$}

From figure 6.2 the reconstructed $B^{ \pm}$mass distribution was fitted with a Gaussian convoluted with a first order polynomial or straight line. The uncertainty on the number of events was calculated from the uncertainty on the amplitude of the Gaussian fit. Therefore the number of $B^{ \pm} \rightarrow J / \psi K^{ \pm}$events observed was $490 \pm 23$. 

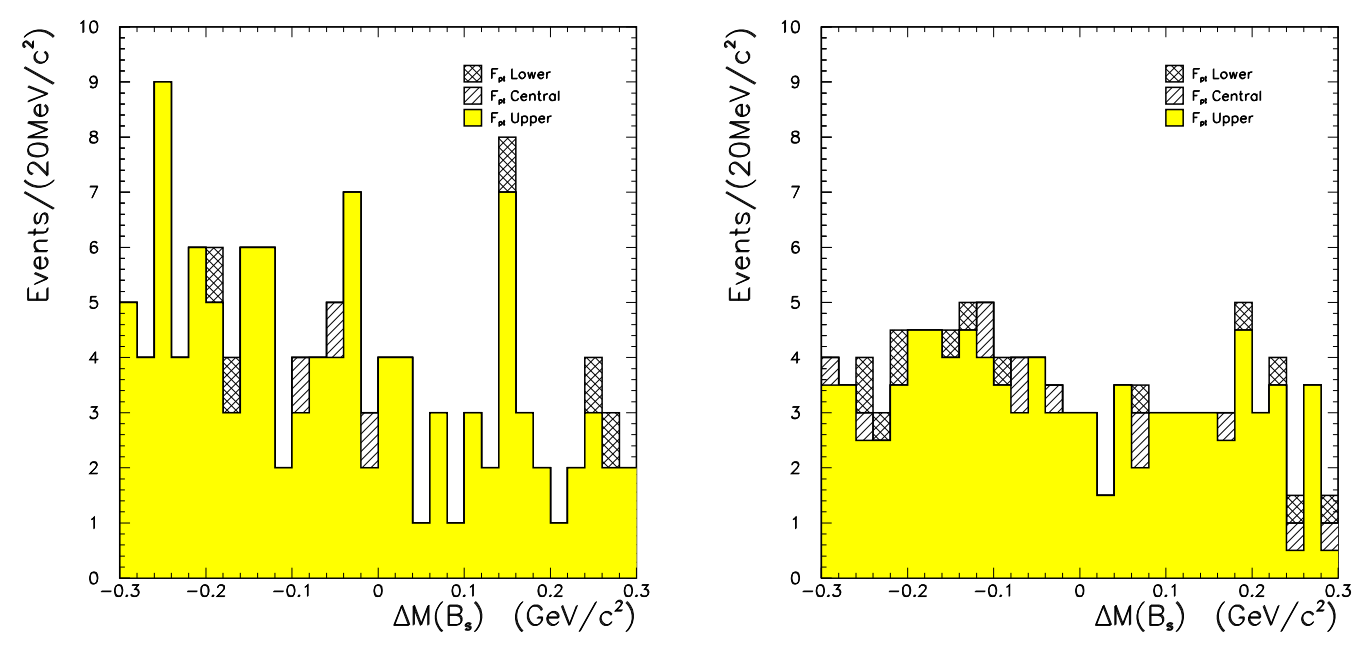

Figure 5.1: Mass distributions for $B_{s}^{0} \rightarrow J / \psi \eta$ candidates: Left $M(\gamma \gamma)$ within $120 \mathrm{MeV}$ of the world average $M(\eta)$, and Right $120 \mathrm{MeV}$ either side of the $M(\gamma \gamma)$ central window. Both plots show the mass distributions with the three limits of $f\left(p_{t}^{J / \psi}\right)$ fit parameters.

\subsubsection{Lifetime Constraints}

The value of $f\left(p_{t}^{J / \psi}\right)$, described in section 4.3.4, was calculated by fitting the distribution illustrated in figure 4.7. Similar polynomial fits were made of the upper and lower error bounds as a function of $p_{t}^{J / \psi}$. To propagate these errors into a $90 \%$ confidence limit, the errors were approximated as Gaussian. Then the value at $90 \%(1.28 \sigma)$ from the mean was used as to calculate $\lambda^{B_{s}^{0}}$ on an event by event basis. The results of applying the $\lambda^{B_{s}^{0}}$ constraint with the three values of $f\left(p_{t}^{J / \psi}\right)$ fit parameters are illustrated in figure 5.1. From the lower, central and upper $1.28 \sigma$ errors the $90 \%$ confidence limit on the number of $B_{s}^{0} \rightarrow$ $J / \psi \eta$ events was calculated. A summary of the uncertainty from $f\left(p_{t}^{J / \psi}\right)$ propagating into the final result is given in table 5.8.

\begin{tabular}{|c|c|c|}
\hline \hline$N\left(B_{s}^{0} \rightarrow J / \psi \eta\right)$ at $90 \%$ C.L. & Lower Variation & Upper Variation \\
\hline 25.9 & $0.4 \%$ & $-5.0 \%$ \\
\hline \hline
\end{tabular}

Table 5.8: Systematic errors from the uncertainty in the $f\left(p_{t}^{J / \psi}\right)$ distribution. 


\subsection{Summary}

All sources of uncertainty are summarised in table 5.9. From this table it is clear that the dominant error influencing the final branching ratio result comes from the external input and in particular $f_{s} / f_{u}$. In comparision to the other sources of uncertainty it is four times greater than any other error involved. The magnitude of this uncertainty suggests that the ratio of the number of $B_{s}^{0} \rightarrow J / \psi \eta$ and $B^{ \pm} \rightarrow J / \psi K^{+}$events is a useful quantity to quote.

Next in the order of influence is the statistical error connected with the number of $B^{ \pm} \rightarrow J / \psi K^{ \pm}$events observed. As more data are taken during Run II this uncertainty will decrease. The uncertainty connected with the CES resconstruction efficiency is close to this statistical error in magnitude. This systematic error enters from uncertainties in the fit to conversion data. The magnitude of this error is internally controlled by the number of conversion electron partners passing the constraints outlined in section 4.4.3. Therefore if the analysis is performed on a larger sample of data at Run II, (including increased material in the silicon volume), this uncertainty will decrease also. This leaves two non-reducable systematic uncertainties: the decay of the $K^{ \pm}$in flight and the lifetime approximations along with other smaller and less significant errors.

Some errors effect the results at a rate below which the simulation is sensitive due to the limited number of monte carlo events generated. These are the uncertainties connected with the level 2 trigger and the primary vertex generation. The maximum error was used as an estimate. Any error in calculating these uncertainties is small enough not to affect the final result. 


\begin{tabular}{|c|c|c|c|}
\hline Source & Reference & Error & Type \\
\hline External & Section 5.2 & $33 \%$ & Systematic \\
\hline B Meson Production $^{\dagger}$ & Section 5.3 .2 & $0.9 \%$ & Systematic \\
\hline Primary Vertex Generation ${ }^{\dagger}$ & Section 5.3.3.1 & $0.01 \%$ & Systematic \\
\hline Level $1 J / \psi$ Trigger $^{\dagger}$ & Section 5.3.3.2 & $0.12 \%$ & Systematic \\
\hline Level $2 J / \psi$ Trigger $^{\dagger}$ & Section 5.3.3.2 & $0.06 \%$ & Systematic \\
\hline$K^{ \pm}$Reconstruction Efficiency & Section 5.3 .4 & $3.1 \%$ & Systematic \\
\hline$K^{ \pm}$Decay In Flight & Section 5.3.4 & $4 \%$ & Systematic \\
\hline CES Reconstruction Efficiency ${ }^{\dagger}$ & Section 5.3 .5 & $1.73 \%$ & Systematic \\
\hline $\mathrm{N}\left(B^{ \pm} \rightarrow J / \psi K^{ \pm}\right)$ & Section 5.4 .1 & $7.96 \%$ & Statistical \\
\hline Lifetime Approximation ${ }^{\ddagger}$ & Section 5.4 .2 & $5 \%$ & Systematic \\
\hline
\end{tabular}

Table 5.9: A summary table of all uncertainties. ( $\dagger$ All of these errors were calculated by varying the associated parameter within the Monte Carlo. $\ddagger$ This error was propagated into the final result by simply picking the highest upper $90 \%$ C.L. i.e. lower limit.) 


\section{Chapter 6}

\section{Results}

\subsection{Introduction}

The results of this thesis are divided up into a repeated analysis to find $B^{ \pm} \rightarrow J / \psi K^{ \pm}$ events, a prediction of the number of $B_{s}^{0} \rightarrow J / \psi \eta$ events within Run I and a final branching ratio $90 \%$ confidence limit for $\mathcal{B}\left(B_{s}^{0} \rightarrow J / \psi \eta\right)$.

\section{$6.2 \quad B^{ \pm} \rightarrow J / \psi K^{ \pm}$}

Following the analysis previously described in section 4.3.3 the CDF data were searched for $B^{ \pm} \rightarrow J / \psi K^{ \pm}$decays. These decays were fully reconstructed in a similar manner to the Monte Carlo described within 4.2.

The proper time distribution for events passing all constraints, except the proper time requirement, was plotted in figure 6.1. The final mass distribution for $B^{ \pm}$events passing all cuts is illustrated in figure 6.2. A Gaussian and a first-order polynomial was fitted to the observed signal to find $490 \pm 23$ events above background.

\subsection{Branching Ratio $\mathcal{B}\left(B_{s}^{0} \rightarrow J / \psi \eta\right)$}

\subsubsection{Events Predicted}

From the number of $B^{ \pm} \rightarrow J / \psi K^{ \pm}$events the predicted number of $B_{s}^{0} \rightarrow J / \psi \eta$ events was calculated by using equation 4.4. From this calculation there should be $2.14 \pm 1.07$ observable $B_{s}^{0} \rightarrow J / \psi \eta$ events in the CDF Run I data. 


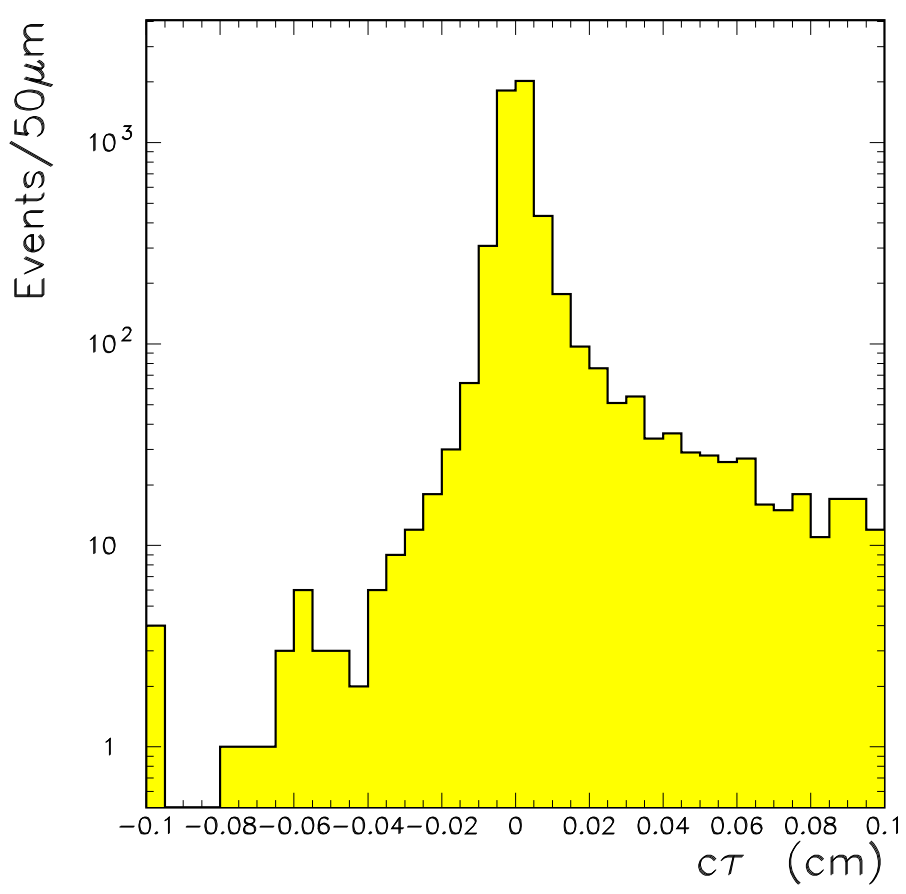

Figure 6.1: The distribution of $c t_{\text {true }}$ for the fully reconstructed $B^{ \pm} \rightarrow J / \psi K^{ \pm}$sample. The data shown passed all of the constraints except the requirement made on $c t_{\text {true }}$ and are within $60 \mathrm{MeV}$ of the world average $B^{ \pm}$mass.

\subsubsection{Events Observed}

The results of the analysis to find $B_{s}^{0} \rightarrow J / \psi \eta$ are shown in figure 6.3. There are more events than the monte carlo prediction shown and no discernible peak. Since it is not possible to detect the decay with CDF Run 1 data a $90 \%$ confidence limit was formed.

\subsubsection{0\% Confidence Limit}

Calculating a Branching Ratio Confidence Limit To calculate a $90 \%$ confidence limit for the branching ratio $B_{s}^{0} \rightarrow J / \psi \eta$ the equation 4.4 was rearranged to form:

$$
\mathcal{B}\left(B_{s}^{0} \rightarrow J / \psi \eta\right)=\frac{f_{u}}{f_{s}} \frac{\mathcal{B}\left(B^{+} \rightarrow J / \psi K^{+}\right)}{\mathcal{B}(\eta \rightarrow \gamma \gamma)} \frac{M C\left(J / \psi K^{+}\right)}{M C(J / \psi \eta)} \frac{N(J / \psi \eta)}{N\left(J / \psi K^{+}\right)}
$$

where $N\left(J / \psi K^{+}\right)$and $N(J / \psi \eta)$ are the number of events observed above background.

The analysis was run to form a $B_{s}^{0}$ mass spectrum for photon pairs within a $120 \mathrm{MeV}$ 


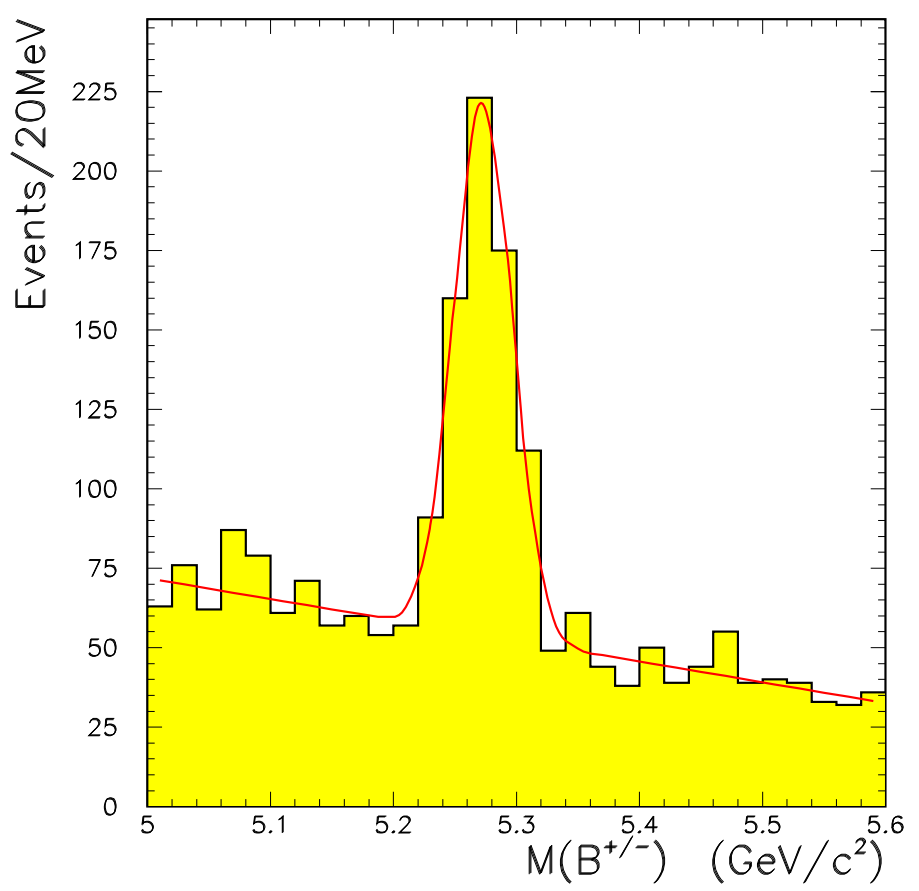

Figure 6.2: The reconstructed $B^{ \pm}$mass distribution from fully reconstructed $B^{ \pm} \rightarrow$ $J / \psi K^{ \pm}$events. From the fit shown there are $490 \pm 23$ events above background.

window of the $\eta$ mass and within the two $120 \mathrm{MeV}$ windows either side of the central window. The $M(\gamma \gamma)$ side bands were used to predict the background. Given a background rate above 10 events, Gaussian statistics can be used. For Gaussian errors the measured value can be related to the $90 \%$ confidence limit by:

$$
x_{\text {meas }}+1.28 \sqrt{x_{\text {true }}}=x_{\text {true }},
$$

where $x_{\text {meas }}$ is the measured number of events within the central $120 \mathrm{MeV}$ window of the $\eta$ mass and $x_{\text {true }}$ is the true value as illustrated in figure 6.4.

Adding in a background

$$
x_{\text {meas }}+b g d+1.28 \sqrt{x_{\text {true }}+b g d}=x_{\text {true }}+b g d,
$$

where $b g d$ refers to the number of events observed using the mean side band region.

Then rearranging the limits can be found by using the general quadratic solution where

$$
a x_{\text {true }}^{2}+b x_{\text {true }}+c=0
$$




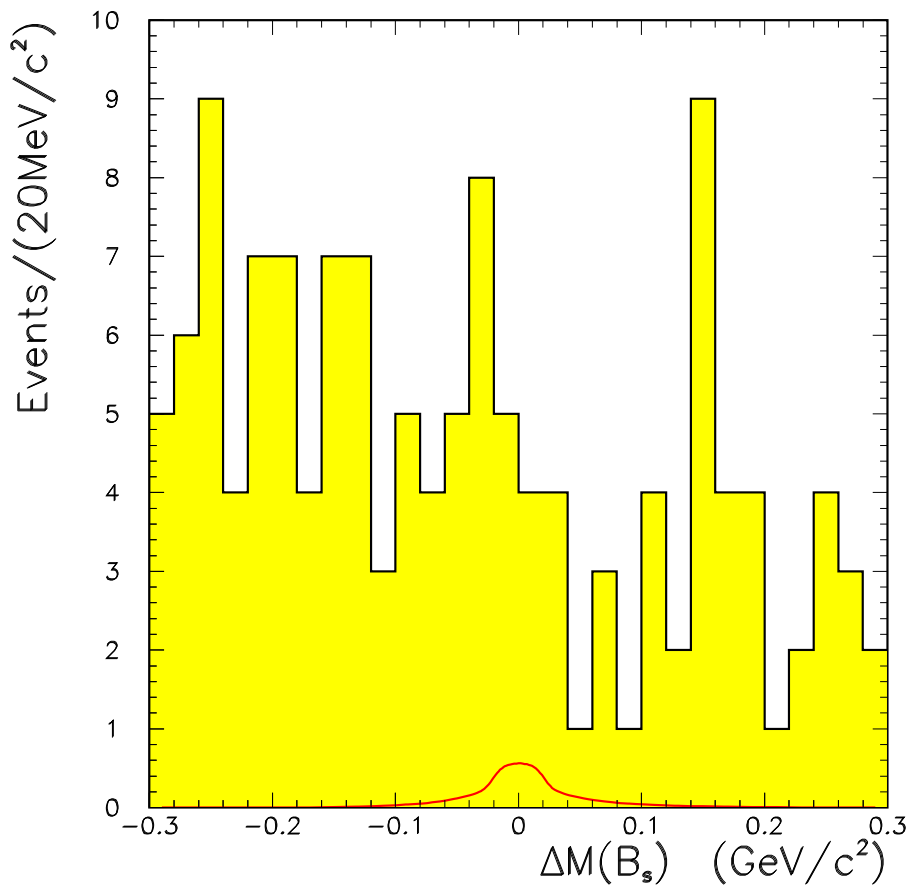

Figure 6.3: The reconstructed $B_{s}^{0}$ mass distribution from $B_{s}^{0} \rightarrow J / \psi \eta$ candidates. The curve is the Monte Carlo distribution renomalised for the predicted number of $B_{s}^{0} \rightarrow J / \psi \eta$ events.

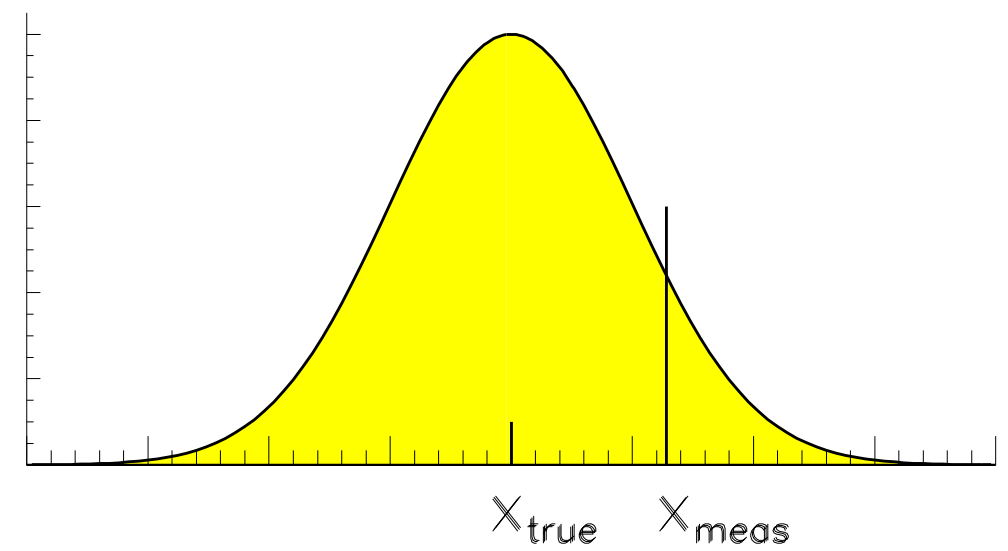

Figure 6.4: An illustration of a 90\% Gaussian confidence limit.

and

$$
a=1.0 \quad b=-2 x_{\text {meas }}-(1.28)^{2} \quad c=x_{\text {meas }}^{2}-(1.28)^{2} b g d
$$


Having found a number of events coinciding to a $90 \%$ C.L. this number was substituted into equation 6.1 as $N(J / \psi \eta)$, and therefore an upper $90 \%$ confidence limit on the branching ratio $\mathcal{B}\left(B_{s}^{0} \rightarrow J / \psi \eta\right)$ was calculated.

Adding in the Errors All uncertainties given in table 5.9, were combined into a total error. This error corresponds to one $\sigma$. Therefore to combine the errors with the branching ratio limit, the total error value at a $90 \%$ confidence limit is $1.28 \sigma$, where $\sigma$ is the total systematic error. The limit was updated to incorporate these errors by adding the error at $90 \%$ confidence. The final value calculated from $\mathrm{CDF}$ data is $\mathcal{B}\left(B_{s}^{0} \rightarrow J / \psi \eta\right)<6.3 \times$ $10^{-3}$ at $90 \%$ C.L.

\subsubsection{Number Ratio $N\left(J / \psi K^{+}\right) / N(J / \psi \eta)$}

Since the branching ratio limit is dominated by the uncertainty connected with $f_{s} / f_{u}$ the number ratio provides a more accurate and easily updatable form of the final result. The ratio of the number of $B^{ \pm} \rightarrow J / \psi K^{ \pm}$events observed, to the $90 \%$ confidence limit of $B_{s}^{0} \rightarrow J / \psi \eta$ events with and without the detector related effects is given in table 6.1.

\begin{tabular}{|c|c|}
\hline \hline$N\left(J / \psi K^{+}\right) / N(J / \psi \eta)$ & 11.5 at $90 \%$ C.L. \\
\hline Corrected $N\left(J / \psi K^{+}\right) / N(J / \psi \eta)$ & 2.50 at 90\% C.L. \\
\hline \hline
\end{tabular}

Table 6.1: A table of number ratios $N\left(J / \psi K^{+}\right) / N(J / \psi \eta)$ with and without the detector related acceptance factors. (Corrected $N\left(J / \psi K^{+}\right) / N(J / \psi \eta) \Longrightarrow$ $\left.M C(J / \psi \eta) / M C\left(J / \psi K^{+}\right) \times N\left(J / \psi K^{+}\right) / N(J / \psi \eta)\right)$

The raw number ratio contains the ratio of detector acceptance factors connected with the CDF Run I detector. This is only useful for approximate CDF Run II predictions. The corrected number ratio can be used by any experiment provided the ratio of acceptance factors are known and can be multiplied back in. 


\section{Chapter 7}

\section{Summary, Conclusions and Prospects}

The number of $B_{s}^{0} \rightarrow J / \psi \eta$ events within $110 \mathrm{pb}^{-1}$ of $p \bar{p}$ at $\sqrt{s}=1.8 \mathrm{TeV}$ was predicted to be $2.1 \pm 1$ in section 6.3.1. Many improvements to the modelling of photon reconstruction within the Collider Detector at Fermilab (CDF) were made. The characteristics of conversion partners and $B_{s}^{0} \rightarrow J / \psi \eta$ decays within the CDF detector were extensively studied in section 4.4.3. The longitudinal shower parameterisation from [35] was used to convert to a calorimeter path independent energy value: removing many differences between $B_{s}^{0} \rightarrow J / \psi \eta$ and conversion partner CES showers. A detailed simulation based on $B^{ \pm} \rightarrow J / \psi K^{ \pm}$data was written to study the effects of ambiguities within the CES clustering. No ambiguities were observed as discussed in section 4.5.3.

From the integrated luminosity of $110 \mathrm{pb}^{-1} 490 \pm 23 B^{ \pm} \rightarrow J / \psi K^{ \pm}$events were isolated, as described in section 6.2. Using the same $J / \psi \rightarrow \mu^{+} \mu^{-}$sample and requiring the same set of constraints on daughter $\mu^{ \pm}$the branching ratio limit $\mathcal{B}\left(B_{s}^{0} \rightarrow J / \psi \eta\right)<6.3 \times$ $10^{-3} 90 \%$ C.L. was set. The dominant systematic error influencing this limit was found to come from the ratio $f_{s} / f_{u}$ as illustrated in section 5.5. The measured branching ratio limit is close to the previous limit of $\mathcal{B}\left(B_{s}^{0} \rightarrow J / \psi \eta\right)<3.8 \times 10^{-3}$ at $90 \%$ C.L.[2], and with future increases in luminosity there is real hope of finding $B_{s}^{0} \rightarrow J / \psi \eta$.

From the data analysed and at a $90 \%$ confidence limit, for every $12 B^{ \pm} \rightarrow J / \psi K^{ \pm}$ events reconstructed during Run I, $1 B_{s}^{0} \rightarrow J / \psi \eta$ event will have been reconstructed and passed all of the constraints used within this thesis as stated in section 6.3.4. Furthermore 
also at a $90 \%$ confidence limit, for every three $B^{ \pm} \rightarrow J / \psi K^{ \pm}$decays there should be one $B_{s}^{0} \rightarrow J / \psi \eta$ event produced by $p \bar{p}$ collisions.

Until the LHC era is well underway, CDF will continue to accumulate the world's most accurate $B_{s}$ data. From the current Run IIA, $2 \mathrm{fb}^{-1}$ of integrated luminosity is expected to be collected. From this large data sample 50,000 $B^{ \pm} \rightarrow J / \psi K^{ \pm}$events are predicted to be observed [5]. Using the values given in chapters 4 and 6 , together with equation 4.4 there should be $218 \pm 100$ observable events within the Run IIA sample. With increased data a ratio of $B_{s}^{0} \rightarrow J / \psi \eta$ and $B_{s}^{0} \rightarrow J / \psi \phi$ may be viable. If this proves to be possible the large systematic error connected with $f_{s} / f_{u}$ would disappear and an accurate measurement of the branching ratio would be possible. 


\section{Appendix A}

\section{Modelling Muon Acceptances}

This Appendix describes details of the $B \rightarrow J / \psi X$ simulation program previously described in section 4.2, with attention placed on the acceptance and reconstruction efficiencies of the muons daughters from the decay. The appendix follows in logical order: discussing the generation of a primary vertex, the geometrical acceptance of CDFs muon chambers and the level 1 and 2 trigger efficiencies.

\section{A.1 Primary Vertex Generation}

The CDF detector, described in chapter 2, has three sets of central muon chambers. In the case of triggering on a $J / \psi \rightarrow \mu^{+} \mu^{-}$decay, the CMU and CMX muon detectors are the most important: previously discussed in section 4.2.4. An overview of the $J / \psi \rightarrow \mu^{+} \mu^{-}$ trigger process is given in chapter 3 .

To properly model a $B \rightarrow J / \psi X$ decay, the decay must be associated with a primary vertex position along the beam axis. For the purposes of muon acceptance, the displacement of the secondary vertex from the primary vertex and the primary vertex distribution with respect to the radial direction, can be neglected. Therefore the $J / \psi \rightarrow \mu^{+} \mu^{-}$vertex was taken as always being at $R=0$ and displaced by some $\Delta z$ from $z=0$, where $R=0$ and $z=0$ were taken as the centre of the detector.

To correctly model the detector effects the primary vertex distribution from $J / \psi \rightarrow$ $\mu^{+} \mu^{-}$data was used to produce a histogram from which Monte Carlo vertices were produced. The $J / \psi \rightarrow \mu^{+} \mu^{-}$vertex distribution along the z-axis is the product of two separate effects acting on a initial Gaussian distribution. These two effects are the 
composite trigger acceptances for the central muon triggers and the acceptance within the silicon detector. The acceptance within the silicon detector enters from the requirement that both muons have three or more silicon hits associated with them: a requirement for good secondary vertex resolution. The distributions of the primary vertex measured from Run I data, after basic $J / \psi$ muon constraints given in table 4.3 , with and without the SVX constraint, are illustrated in figure A.1.
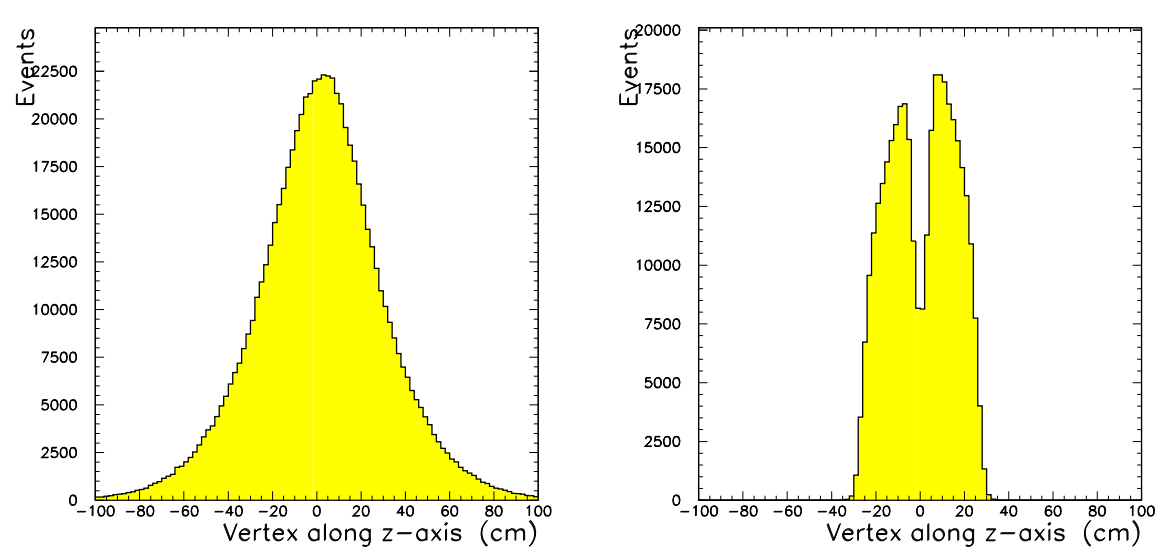

Figure A.1: Primary vertex distribution after basic muon constraints: left without SVX $\left({ }^{\prime}\right)$ constraints, right with three or more hits required on each muon leg.

Using simulation methods described in section 4.2 together with the $J / \psi \rightarrow \mu^{+} \mu^{-}$ trigger constraints, the acceptance of the level 1 and level 2 triggers was measured using a flat primary vertex distribution along the z-axis. The results of this study are given in figure A.2.

Having found the SVX acceptance for $J / \psi \rightarrow \mu^{+} \mu^{-}$the distribution can be used to generate a vertex position along the z-axis. Following the production of $B \rightarrow J / \psi X$ at a given point along the beam axis, the geometric acceptance of the muons chambers was applied, using methods described in section A.2

\section{A.2 CMU and CMX Geometrical Acceptance}

The CMU chambers are parallel to the beam line whereas the centre of the CMX chambers are at a $\theta$ of $48.25^{\circ}$, (measured at the centre of the detector). Both chambers are rectangular in the $r-z$ plane, but for the purposes of this model are assumed to be 

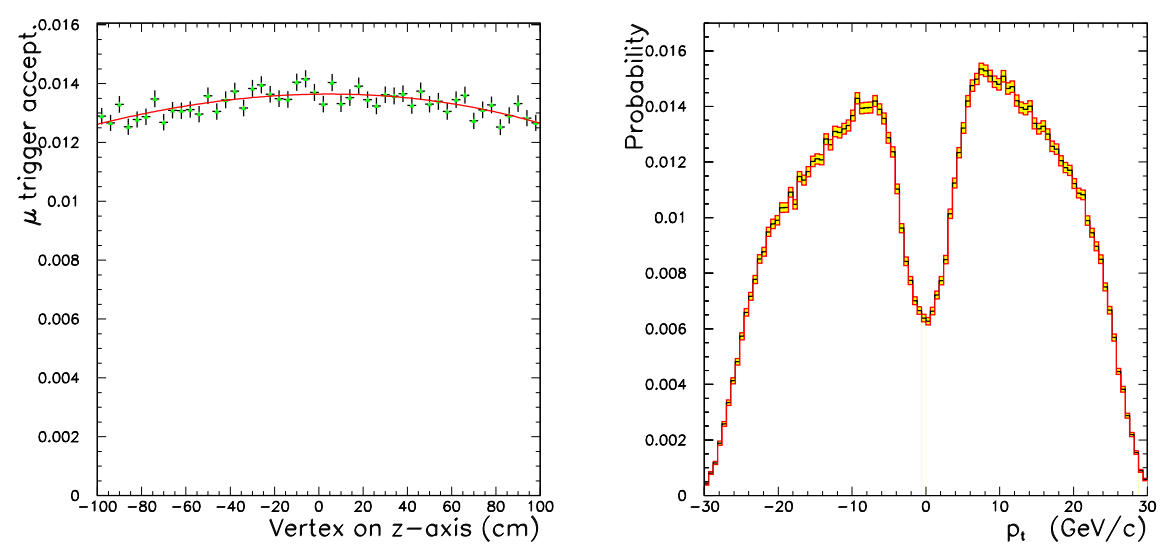

Figure A.2: Left Primary vertex acceptance after geometric acceptance, level 1 and level 2 trigger simulations have been applied. (Each event contained two muons from a $B_{s}^{0} \rightarrow$ $J / \psi \eta$ decay. No $p_{t}$ cut on the $B_{s}^{0}$.); Right The histogram used to generate primary vertices with associated one sigma errors.

described by their inner surface. Generalising both chambers can be thought of as covering some length along an $L$-axis, from $L_{1}$ to $L_{2}$. If the muon track is found to point inside the region described by the points $L_{1}$ and $L_{2}$, then it is geometrically accepted. Since the CMU chamber is the simplest case this was considered first.

\section{A.2.1 CMU Acceptance}

From figure A.3, the position along the L-axis at which a given muon track intersects is given by:

$$
L=\frac{R_{C M U}}{\tan (\theta)}+\Delta z
$$

\section{A.2.2 CMX Acceptance}

The CMX case is more complicated. To turn the problem into something similar to the CMU case, the perpendicular distance from the CMX axis to the primary vertex $z$ position was calculated from figure A.4.

Using simple trigonometry the distance $r^{\prime}$ can be expressed as:

$$
r^{\prime}=R_{C M X}-\Delta z \cos \left(\theta_{C M X}\right)
$$




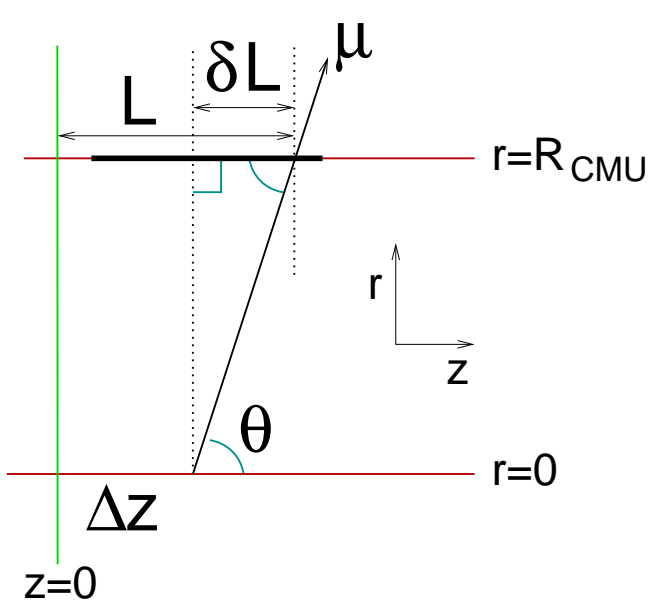

Figure A.3: A muon track in the $r-z$ plane, intersecting the CMU axis.

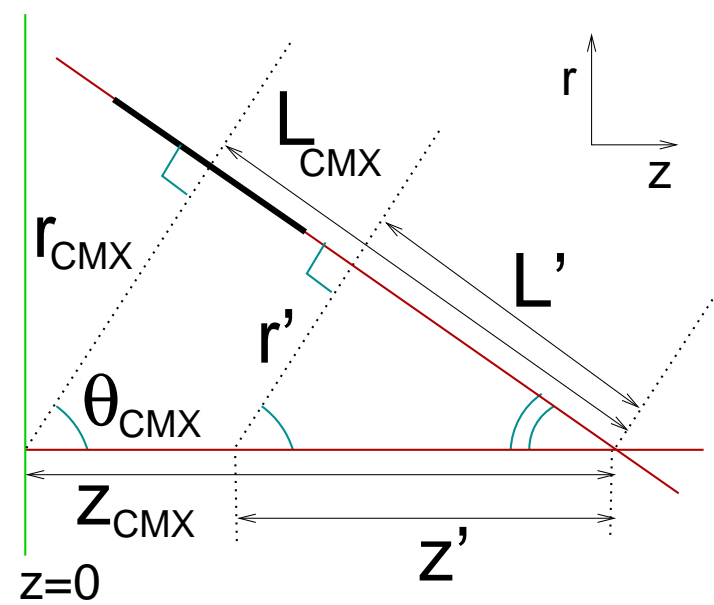

Figure A.4: Perpendicular distance from the CMX axis to the primary vertex in the $r-z$ plane

where $R_{C M X}$ is the radius at which the CMX is position, and $\Delta z$ corresponds to the generated primary vertex z position.

Using the value of $r^{\prime}$ from equation A.1, the point of intersection with the CMX axis can be calculated as:

$$
L=L^{\prime}+\delta L=r^{\prime} \tan \left(\theta_{C M X}\right)+r^{\prime} \tan \left(\theta-\theta_{C M X}\right)
$$

where $\delta L$ is illustrated in figure A.5. 


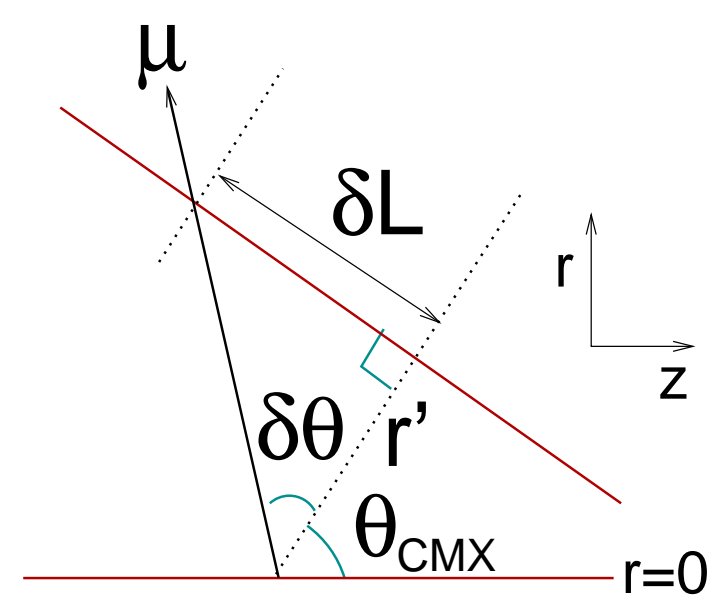

Figure A.5: An illustration of $\delta L$ in the $r-z$ plane

\section{A.3 Level 1 Muon Trigger Acceptance}

The level 1 muon trigger acceptance was measured by using the single low $p_{t}$ muon trigger. Some dimuons were accepted into this sample. After reconstruction they can be found by recombining tracks with the trigger accepted muon. Pairs that form the $J / \psi$ mass have one muon which is effected by the trigger acceptance and one that is not. This second muon can be used to calculate the efficiency of reconstruction within a muon chamber. This is a simple matter of plotting the $p_{t}$ spectrum of the un-biased muon before requiring a dimuon trigger flag and after requiring a dimuon trigger flag. Then the division of the two histograms will yield the efficiency of reconstruction as a function of $p_{t}$ of the muon track. The procedure is described in length in [24].

To simulate the effects of the detector this curve was parameterised for use in the DIMUTG package [48]. Unfortunately the CMX level 1 trigger acceptance was never measured but should be approximately the same shape as the CMX response. Therefore the CMU parameterisation was used for the CMX together with a renormalisation to account for the observed difference in rate between the two triggers. The two normalised curves used in the calculation were plotted in figure A.6. respectively. 

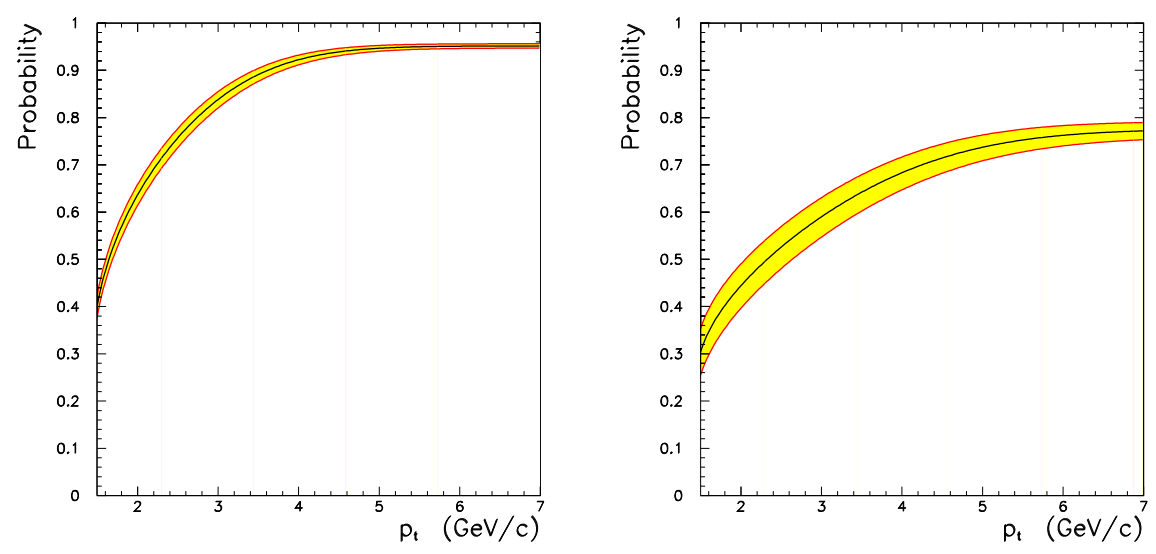

Figure A.6: Level 1 trigger efficiencies as a function of transverse momentum $\left(p_{t}\right)$ : left $\mathrm{CMU}$, right CMX. The errors either side of the mean were extracted from varying the fit parameters within one sigma of each associated error.

\section{A.4 Level 2 Muon Trigger Acceptances}

For the level 2 muon trigger acceptance, the parameterised efficiency curves from DIMUTG [48], were used. The level 2 trigger efficiency curves were extracted in a similar manner to the level 1 trigger curves described in section A.3. Events were collected from CMU and CMX level 2 trigger samples were only one CFT track was required. The $p_{t}$ of the unconstrained muon was plotted before and after requiring a second CFT trigger. The ratio of the two plots was used to calculate the efficiency of the CFT match with respect to $p_{t}$. Fits to these efficiency curves for the CMU and CMX regions where used to calculate the level 2 muon trigger acceptance. Although azimuthal, and charge dependence are available the effects are small and for Monte Carlo it is simplest to use the average curve. The curved and the associated one sigma variations of the fit parameters are shown in figure A.7. 

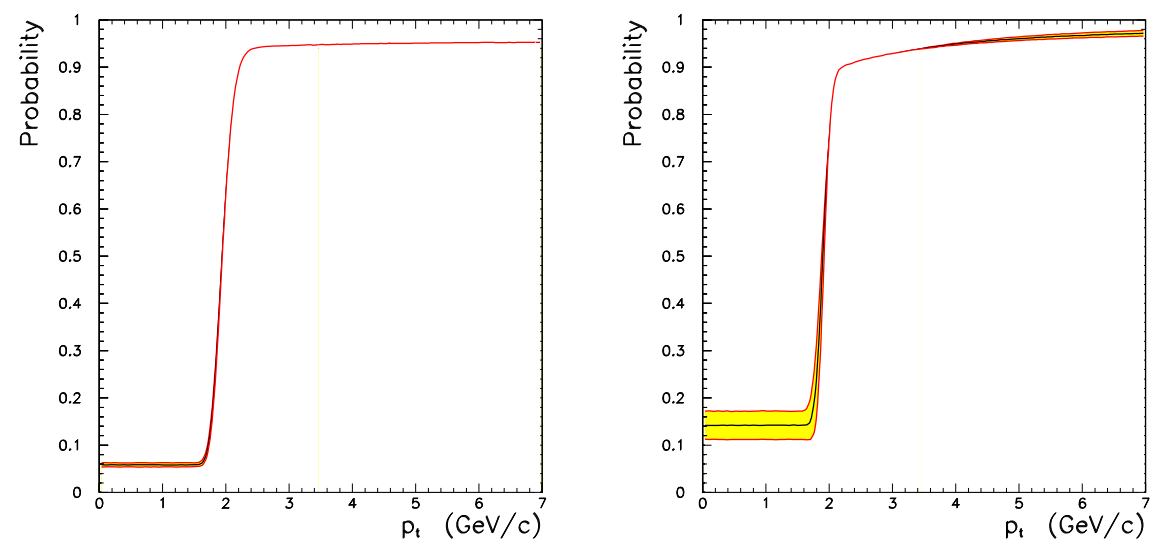

Figure A.7: Level 2 trigger efficiencies for a CFT match as a function of transverse momentum $\left(p_{t}\right)$ : left CMU, right CMX. The errors either side of the mean were extracted from varying the fit parameters within one sigma of each associated error. Azimuthal and pseudorapidity effects have been taken at their mean values. 


\section{Appendix B}

\section{Proportional Strip Chamber Clustering}

The CES as mentioned in section 2.3.3.1, is split into two halves. Each half chamber contains wires running along the beam axis and strips running perpendicular to them. Each calorimeter tower contains a number of strips. At the boundary of the two halves of the chamber the anode wires are terminated, i.e. the two halves of the chamber have two sets of 32 wire channels. In normal use the CES chamber can be used to refine an electron sample by requiring the presence of an electromagnetic shower, which passes some $\chi^{2}$ fits in both views. With photons there clearly isn't any helical track to trace into the CES. Low energy photons rarely leave any signal in the preshower detector. Therefore the CES must be used without any prior knowledge. To do this as efficiently as possible cluster matching must be done with care. If all showers produce CES clusters with the same profile then the strip-wire matching could be done on the basis of the central channel, thus optimising the area. However, clustering where matches are made based on just one CES channel are prone to ambiguity. To improve the situation, but still use a lot of the CES area, threechannels were used. Assuming three-channel clustering, the clustering area available is smaller than the total area available because the central channel is required to be one channel from the edge of the half chamber.

The cluster finding implemented used the same logic for both strip and wire channels. Corrections exist for strip and wire gain ratios [56] but have to be ignored at this stage because apriori a strip cluster could be part of any wire cluster, and the strip/wire gain 
map relies on knowledge of the $z, x$ position for a correction to be made. Clustering without gain correction could cause ambiguities and is discussed in section 4.5. To find a cluster in any view a simple algorithm was employed. The algorithm finds all the local maxima within both the strip and wire views. The maxima were selected taking into account the noise associated with strip and wire channels. Having found all the local maxima the three-channel sum of the maxima and the two side channels was stored. The list of maxima for the two views were then sorted into ascending order. The sorted lists were then paired from the top down i.e. the two largest clusters were assumed to be the same shower and so on. Any unmatched strips were recorded but flagged as unmatched. Noisy channels within the CES module have a small amplitude and tend to be unmatched.

If a calorimeter module contained a candidate tower inside it then the CES chamber behind the calorimeter module was reconstructed. The CEM tower boundaries at the surface of the CES were used to decide which strip clusters were inside the tower. The possible outcomes were: no CES cluster within the tower, a matched CES cluster or more than one cluster within the tower. Un-matched CES clusters were used to determine if there were multiple clusters in the tower but by themselves were not counted as a cluster, i.e. towers with just an unmatched strip cluster were said to not have a CES cluster behind them.

The result of the cluster matching based on raw channel information was then returned so that a fitted position could be found. For each candidate tower containing a matched cluster the central maxima for strip and wire views were used as the seeds for a cluster shape fit. The cluster shape fit used the central channel and three-channels either side if they were available. The final integrated charge recorded by the CES was corrected for chamber gain effects as prescribed in [56]. 


\section{Appendix C}

\section{A study of photon acceptances}

\section{C.1 Introduction}

During $p \bar{p}$ interactions the CDF detector environment is populated with photon backgrounds. These photon backgrounds peak at lower energies. To remove as much background as possible but remain sensitive to $B_{s}^{0} \rightarrow J / \psi \eta$ decays a constraint on the $E_{t}$ on each photon forming a $\eta \rightarrow \gamma \gamma$ candidate had to be carefully set. The following appendix contains a discussion of the calorimeter performance and backgrounds; concluding with the $E_{t}$ constraint applied to photon candidate pairs during the analysis, described in chapter 4 .

\section{C.2 Previous Publications}

Previous analyses on radiative decays within the CDF detector [21] and [19], have used two $E_{t}$ constraints on photon candidates: $0.7 \mathrm{GeV}$ and $1.0 \mathrm{GeV}$. Both of these analyses involved a single photon and were therefore less sensitive to this constraint. More similar to this analysis, some work was done to reconstruct low energy $\pi^{0}$ particles [23] where the $E_{t}$ cut was set to $1.0 \mathrm{GeV}$. Within all of these analyses no systematic reasoning behind the value $E_{t}$ constraint used was discussed. Therefore this appendix is focused on exploring some of the reasons for choosing different values of $E_{t}$. 


\section{C.3 Kinematics and Acceptance}

Before examining detector specific behaviour and areas of systematic error, the fundamental properties of photons produced from $B_{s}^{0} \rightarrow J / \psi \eta$ daughters were looked at. A Monte Carlo sample of $B_{s}^{0} \rightarrow J / \psi \eta$ decays was generated using the methods described in chapter 4 . Then $J / \psi$ daughters were required to pass the level 1 and 2 trigger, discussed in chapter 4 . Without making any further constraints the $E_{t}$ of the two photons was plotted in figure C.1. This figure shows the generated events have a most probable $E_{t}\left(\gamma_{1}\right)+E_{t}\left(\gamma_{2}\right)$ of $0.7 \mathrm{GeV}$. Thus a lower constraint on the calorimeter towers would appear favourable.

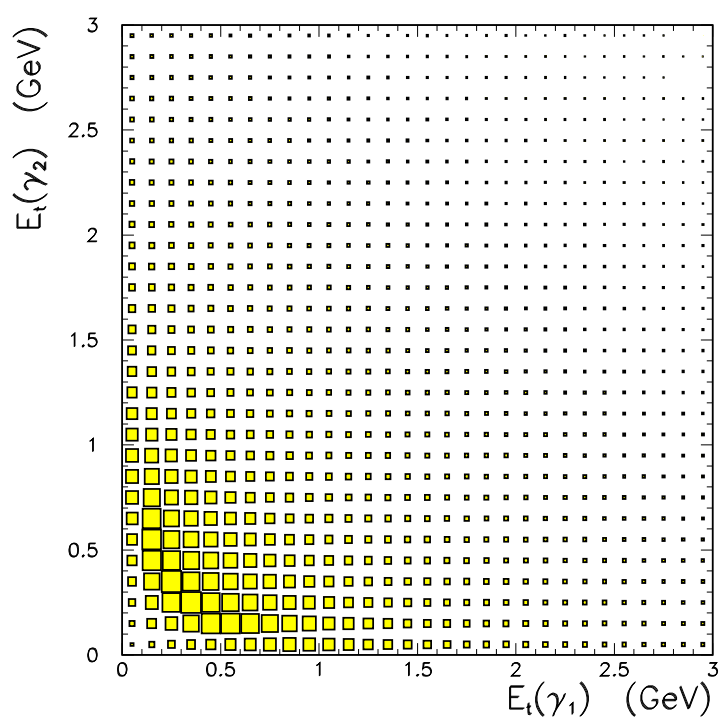

Figure C.1: Photon daughters from $B_{s}^{0} \rightarrow J / \psi \eta$ produced using NLO QCD, MRST99 (central gluon), and Peterson fragmentation. $J / \psi$ trigger constraints have been made.

Re-expressing the information illustrated in figure C.1, the acceptance of $B_{s}^{0} \rightarrow J / \psi \eta$ events after muons trigger effects was plotted. During the analysis a $E_{t}$ constraint was made on every candidate calorimetry tower i.e. the constraints on both photons were the same. The resulting acceptance with respect to the $E_{t}$ requirement made was plotted in figure C.2. Within this figure the photon acceptance changes its curvature slightly before reaching $100 \%$ because there is an effective $p_{t}$ cut on the $\eta$ from the trigger constraints on the $J / \psi$ muons. 


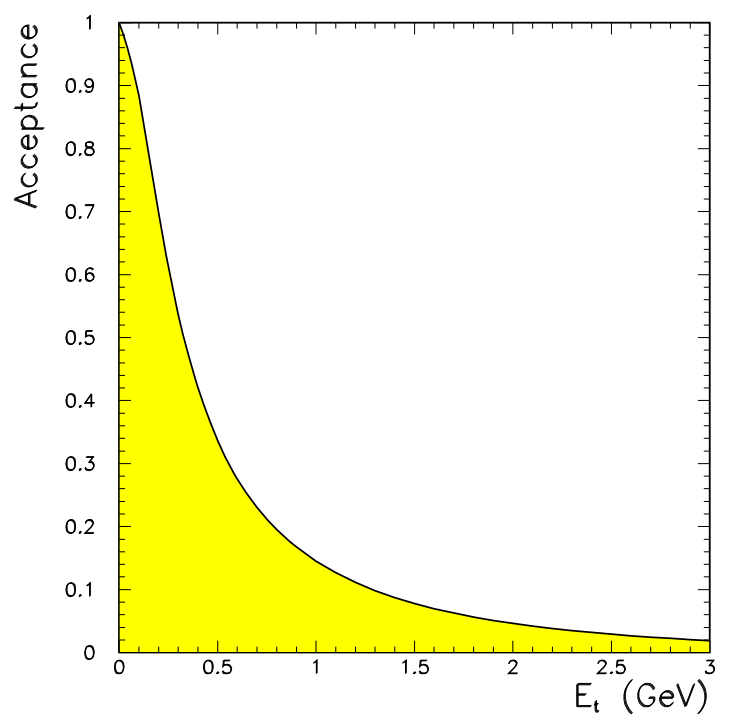

Figure C.2: Acceptance of $B_{s}^{0} \rightarrow J / \psi \eta$ where $\eta \rightarrow \gamma \gamma$, as a function of the calorimeter energy cut made on both photons.

\section{C.4 Calorimeter Performance Effects}

There are two detector related factors which effect the measurement of a low energy $\eta \rightarrow \gamma \gamma$ signal: the calorimeter energy resolution and the CES reconstruction efficiency measurements.

\section{C.4.1 Calorimeter Energy Resolution}

Assuming the CEM calibration is valid below the lowest calibration point of $5 \mathrm{GeV}$, the energy resolution was plotted in figure C.3. Since the width of the $B_{s}^{0}$ mass reconstructed from $B_{s}^{0} \rightarrow J / \psi \eta$ is approximately only due to the electromagnetic energy resolution, the inclusion of low energy photons imply a wider $B_{s}^{0}$ mass distribution. As the mass distribution becomes wider the statistical significance above the background is lower.

\section{C.4.2 CES Reconstruction Efficiency}

The CES efficiency study is described in chapter 4. It used conversion electron partners. These conversion electron partners being charged tracks are not useful below $400 \mathrm{MeV}$ since they do not have enough energy to leave the tracking volume. Therefore the reconstruction 

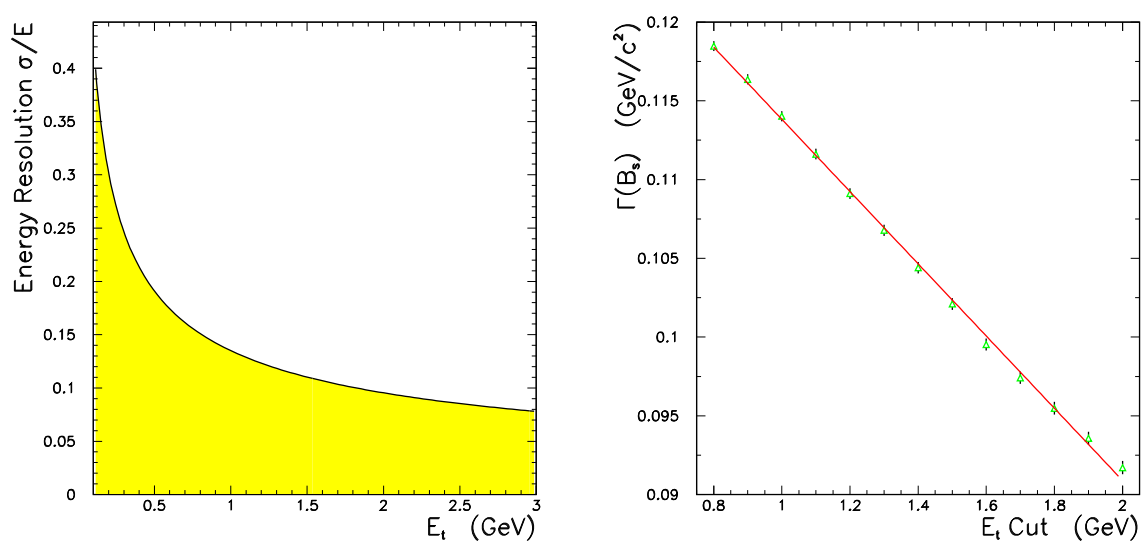

Figure C.3: left CEM calorimeter energy resolution as a function of $E_{t}$. The calorimeter resolution $13.5 \% / \sqrt{E}$ was taken from [9]. $\operatorname{right} \Gamma\left(B_{s}^{0}\right)$ as a function of the $E_{t}$ cut, assuming the width is only due to the electromagnetic calorimeter resolution.

efficiency below $0.5 \mathrm{GeV}$ can only be determined by extrapolation. Combined with this increase in uncertainty is the lower reconstruction probability demonstrated in chapter 4 .

\section{C.5 Diphoton background}

The main source of this background is the $\pi^{0} \rightarrow \gamma \gamma$ decay. If an event contains two $\pi^{0} \rightarrow \gamma \gamma$ decays, then forming an invariant mass plot from all pairs of photons will result in two masses around the $\pi^{0}$ distribution and two higher masses. The behaviour of the $M(\gamma \gamma)$ mass spectrum with respect to the constraint made on $E_{t}$ is illustrated in figure C.4.

From the $M(\gamma \gamma)$ mass distributions shown in figure C.4 together with an addition histogram made using a $1.2 \mathrm{GeV} E_{t}$ cut, the summary plots shown in figure C.5 were plotted. 

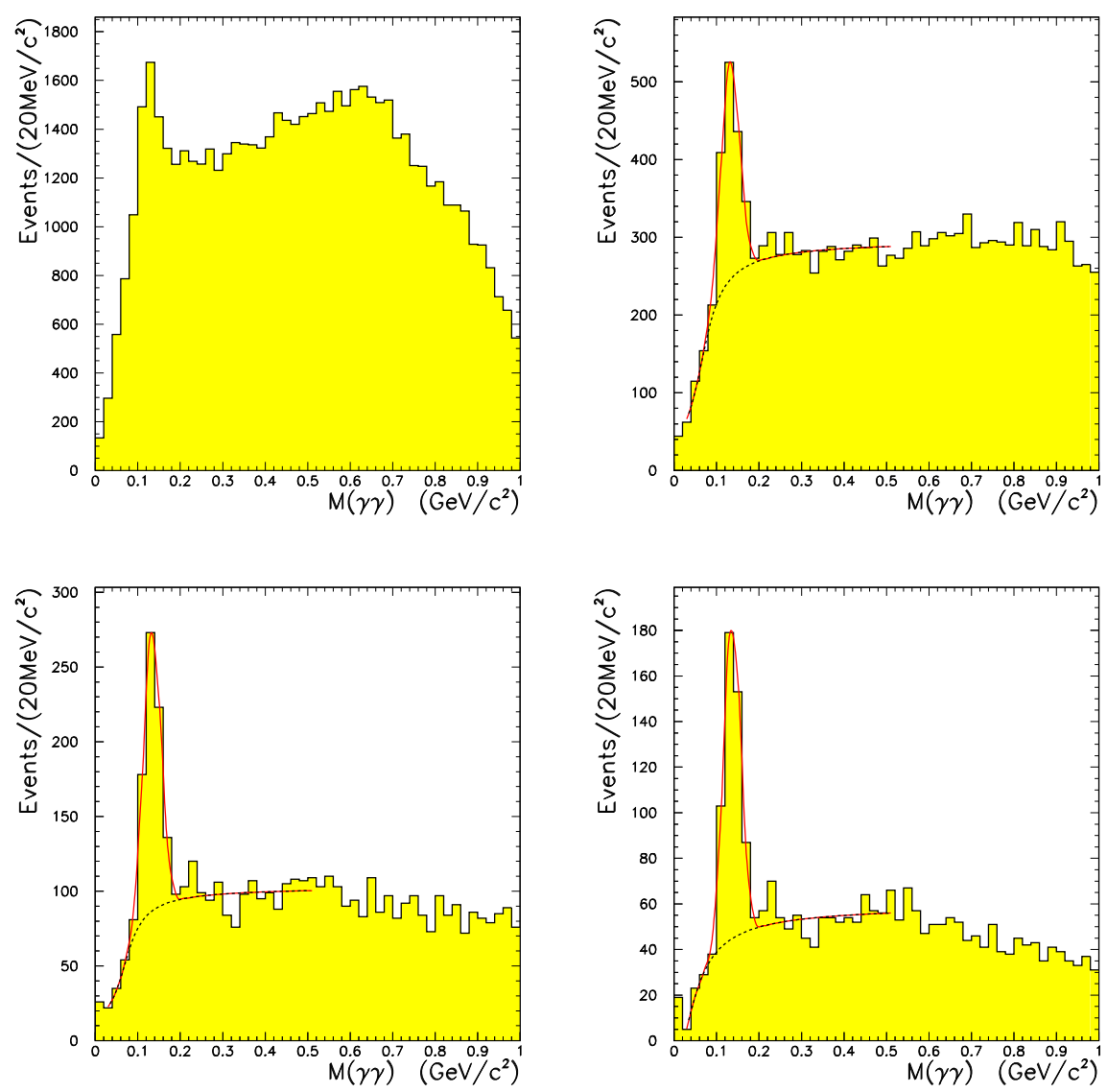

Figure C.4: $M(\gamma \gamma)$ mass distribution for different $E_{t}$ constraints. From the top left to the bottom right, the distributions were made from $J / \psi$ stream B data requiring $E_{t}$ to be greater than $0.4,0.6,0.8$ and $1.0 \mathrm{GeV}$ respectively. 

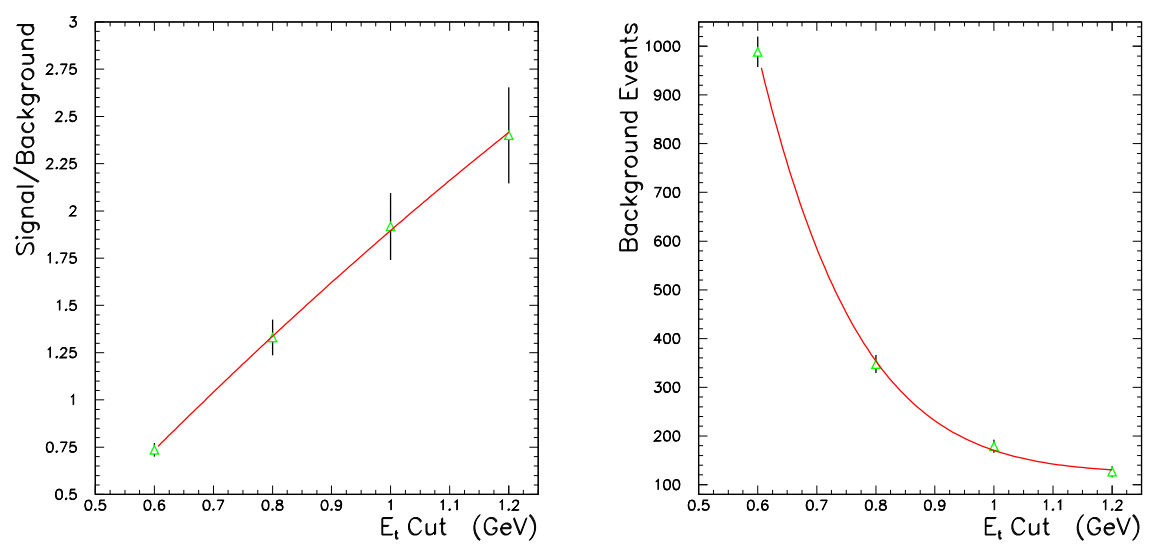

Figure C.5: Summary plots showing the behaviour of the $\pi^{0}$ peak within the $M(\gamma \gamma)$ mass distribution. left Signal/background for the $\pi^{0}$ peak as a function of the $E_{t}$ requirement. right The number of background events under the $\pi^{0}$ mass peak. 


\section{Appendix D}

\section{Conversion Electron Selection Cuts}

\section{D.1 Introduction}

This appendix contains plots of all constraints used to identify and filter conversion pairs from an identified electron sample. These conversion pairs were used, as described in chapter 4 , to determine the CES reconstruction efficiency. The sample by its very nature is a mixture of electrons and positrons. The same constraints were applied to both types of particle.

\section{D.2 Pair Selection}

Conversion partners were identified by their vertex displacement at the tangent point and the difference of the two $\cot (\theta)$ values. The effect of both of these constraints is shown in figure D.1.

\section{D.3 Identified Electron Track}

Identified electrons that were part of pairs passing the selection criterion from section D.2, were required to pass all of the constraints given in table 4.7. The effect of each of these cuts is shown in figures D.2, D.3 and D.4. 

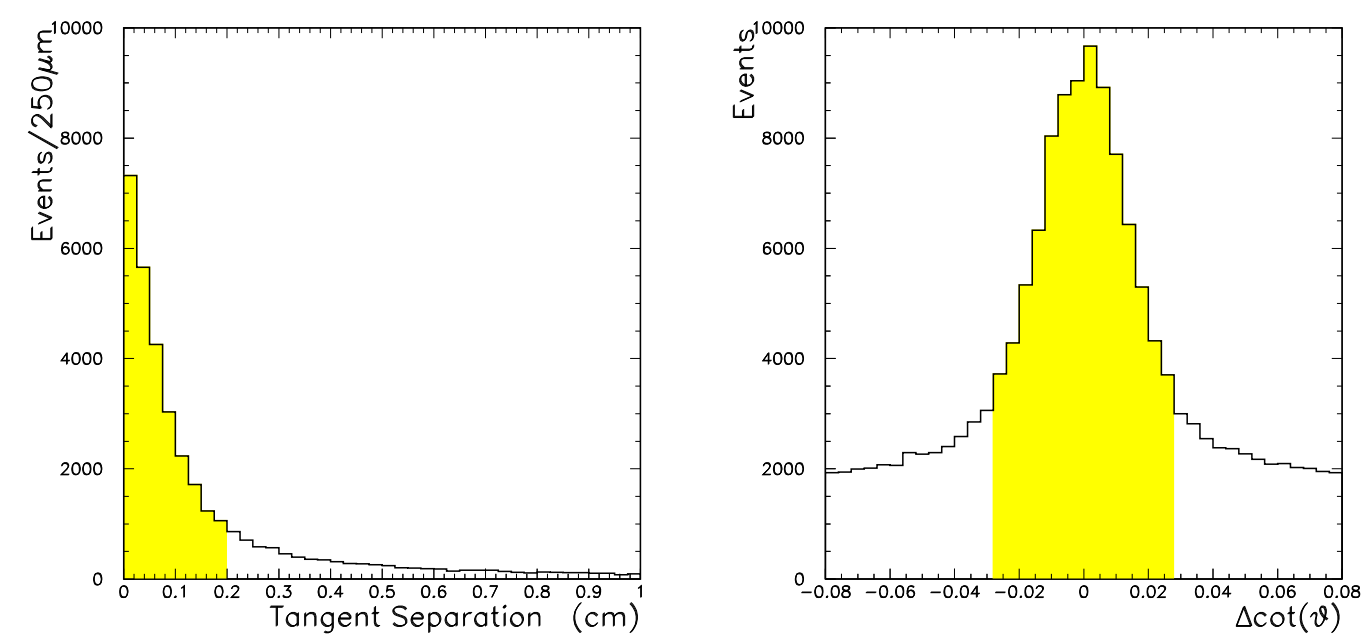

Figure D.1: Left: the distribution of tangent point values, showing the selected region in yellow. Right: the distribution of $\Delta \cot (\theta)$ values, showing the selected region in yellow.
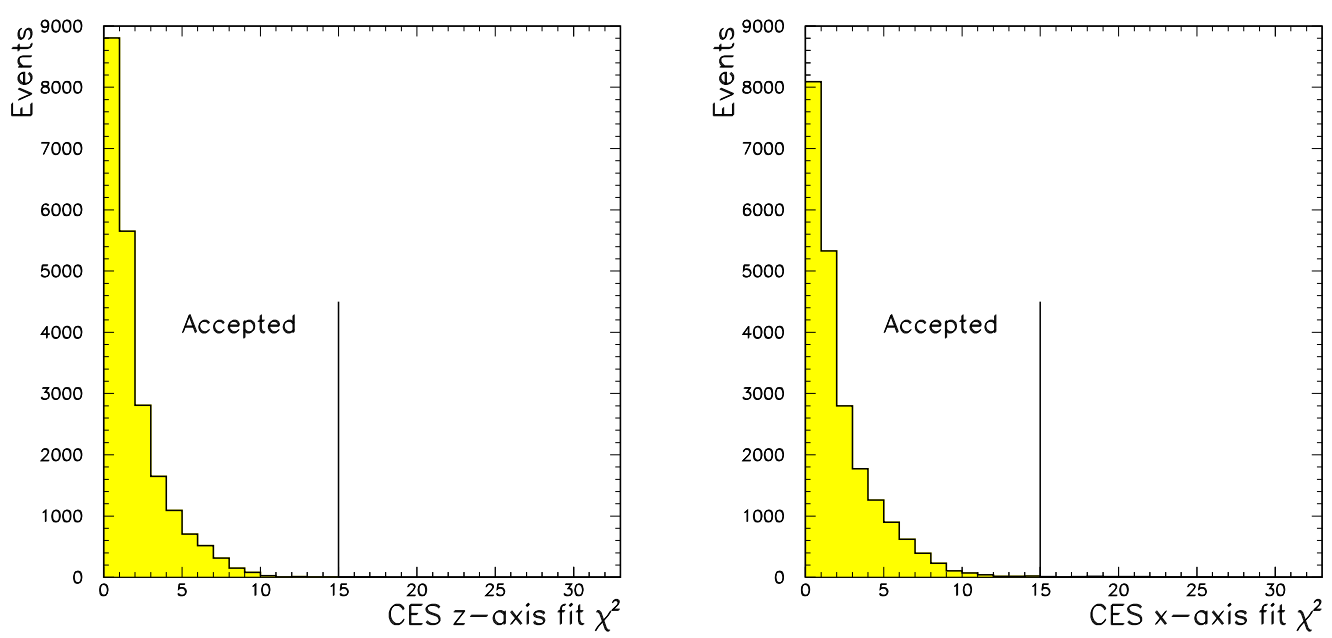

Figure D.2: Left: CES strip fit $\chi^{2}$, showing the selected region in yellow. Right: CES wire fit $\chi^{2}$, showing the selected region in yellow.

\section{D.4 Conversion Partner Electron}

Conversion partners of an identified electron track satisfying all of the cuts within section D.3 were filtered according to the constraints in table 4.8. The range of events selected by each of these constraints is displayed within figures D.5 and D.6. 


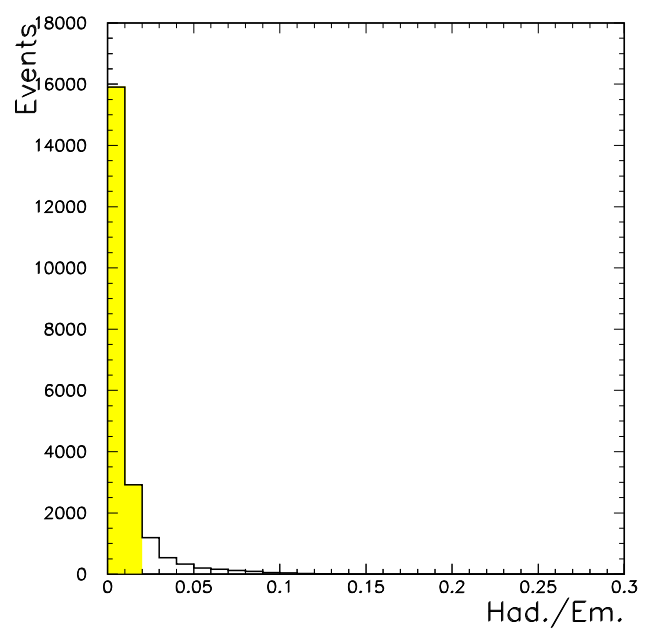

Figure D.3: Hadronic/Electromagnetic energy for the identified electron. The selected region is shown in yellow.
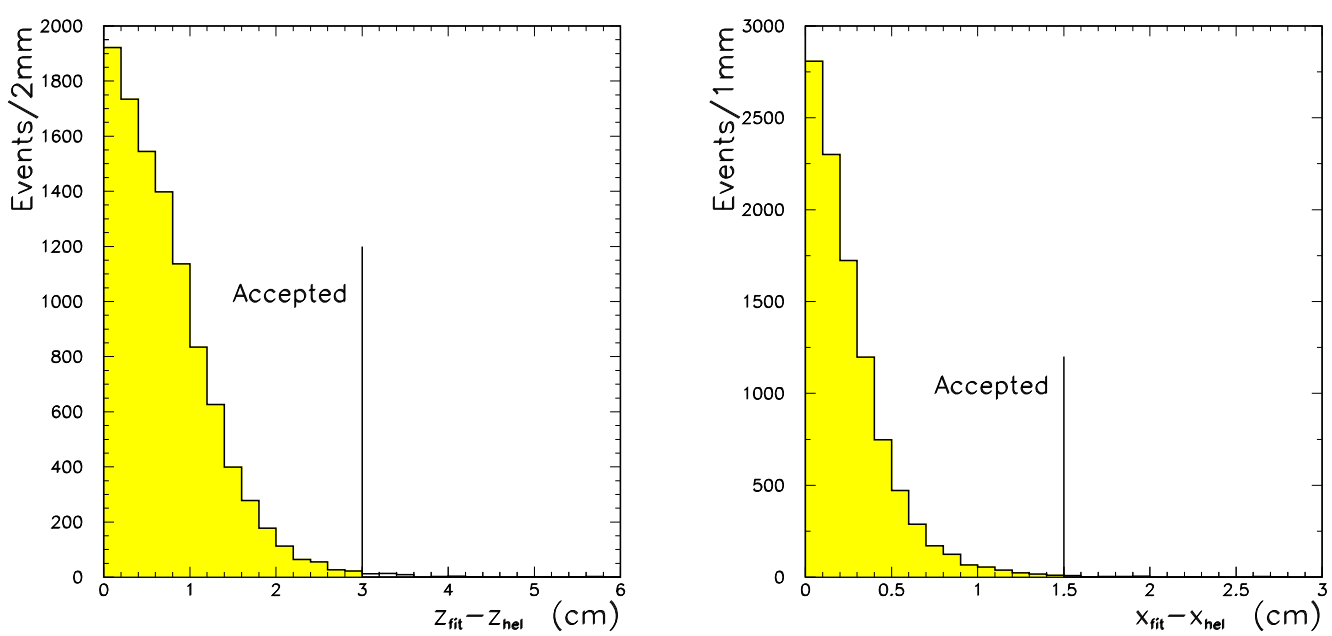

Figure D.4: Left: $z_{f i t}-z_{h e l}$, showing the selected region in yellow. Right: $x_{f i t}-x_{h e l}$, showing the selected region in yellow. 


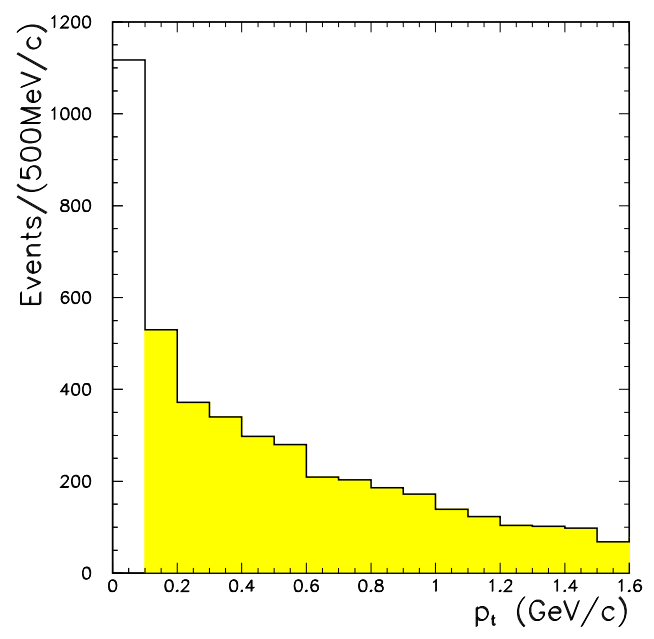

Figure D.5: The $p_{t}$ spectrum of the conversion partner. Events within the selected region are shown in yellow.

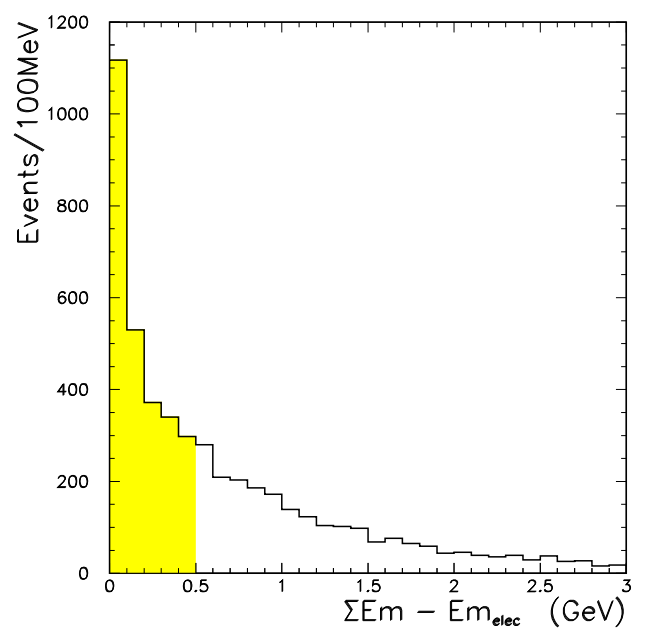

Figure D.6: Background electromagnetic energy within the conversion partners CEM module. (This plot was formed by summing the energy within the tower and then removing the energy of the conversion electron partner.) Events that fall within the selected region are shown in yellow. 


\section{Appendix E}

\section{Tracking Parameters}

As a charged track traverses the tracking volume it does so across a 1.4 Tesla magnetic field acting along the beam axis. This solenoid field not surprisingly causes tracks to travel in helical obits. A single track is illustrated in figure E.1, showing the definitions of key tracking parameters.

The angles $\phi_{0}$ and $\theta$ are related to the four momentum components by:

$$
\begin{gathered}
\phi_{0}=\tan ^{-1}\left(\frac{p_{y}}{p_{x}}\right) \\
\cot \theta=\frac{p_{z}}{p_{t}}
\end{gathered}
$$

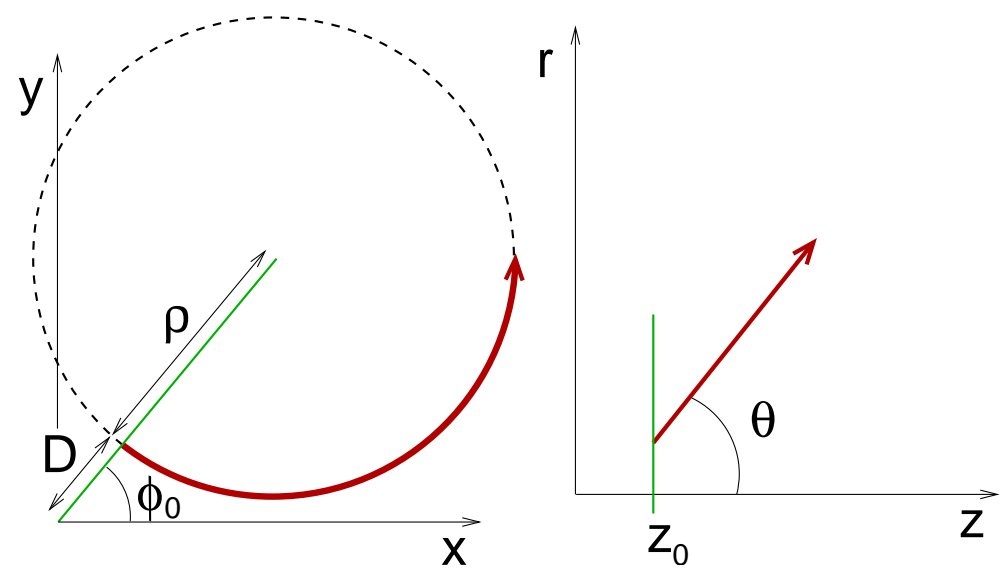

Figure E.1: A helical track within two, two dimensional planes: where $\rho$ is the radius of the helix and $D$ is the impact parameter. 
The radius is related to the curvature $C$ by:

$$
\rho=\frac{1}{2 C}
$$




\section{Glossary}

\section{Transverse Momentum $\left(p_{t}\right)$}

$$
p_{t}=\sqrt{p_{x}^{2}+p_{y}^{2}}
$$

Where $p_{x}$ and $p_{y}$ refer to the $\mathrm{x}$ and $\mathrm{y}$ components of momentum respectively.

\section{Rapidity (y)}

$$
y=\frac{1}{2} \ln \left(\frac{E+p_{z}}{E-p_{z}}\right)
$$

Where $E$ refers to the total energy of a particle and $p_{z}$ refers to the $\mathrm{z}$ momentum component. The shape of the rapidity distribution is invariant under a Lorentz boost along the z-axis[33].

\section{Pseudo-rapidity $(\eta)$}

$$
\eta=-\ln [\tan (\theta / 2)]
$$

A good approximation to rapidity for highly relativistic particles where $p \gg m[33]$.

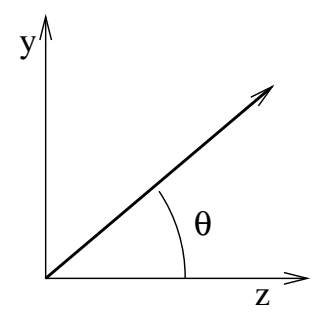

Integrated Luminosity $(\mathcal{L})$ The event rate $R$ in a collider is proportional to the interaction cross section $\sigma_{i n t}$ and the factor of proportionality is called the luminosity.

$$
R=L \sigma_{i n t}
$$


If two bunches containing $n_{1}$ and $n_{2}$ particles collide with frequency $f$, then the luminosity is:

$$
L=f \frac{n_{1} n_{2}}{4 \pi \sigma_{x} \sigma_{y}}
$$

where $\sigma_{x}$ and $\sigma_{y}$ characterise the Gaussian transverse beam profiles in the horizontal and vertical directions. Integrated Luminosity refers to:

$$
\mathcal{L}=\int L d t
$$

Vertex Displacement $\left(L_{x y}\right)$ The secondary vertex displacement from the primary vertex measured in the $\mathrm{x}-\mathrm{y}$ plane. During CDF Run I analyses the vetex displacement

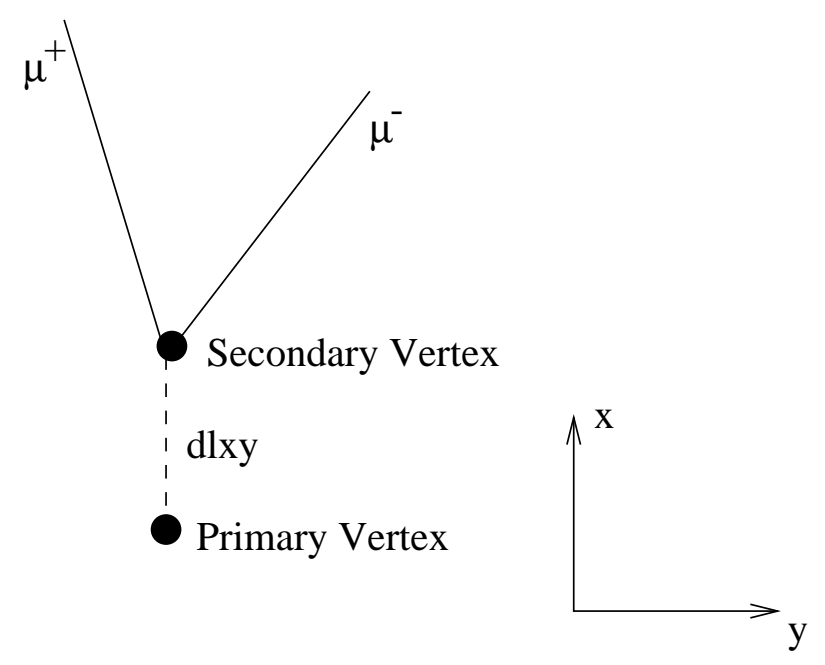

within the plane was used instead of the three dimensional displacement. (The simple relation $L_{x y} / p_{t}=L_{x y z} / p$ was used.) 


\section{Bibliography}

[1] F. ABe Eт AL., Evidence of Top Quark Production in $p \bar{p}$ Collisions at $\sqrt{s}=1.8$ TeV, Phys. Rev. D, 50 (1994), p. 2966.

[2] M. ACCIARRI Et AL., Search for exclusive $b$ decays to $J / \psi$ and $\eta$ or $\pi^{0}$ with the L3 detector, Phys. Lett., B391 (1997), p. 481.

[3] D. Amidei et AL., A Two Level FASTBUS Based Trigger System for CDF, Nucl. Instr. Meth., A269 (1988), p. 51.

[4] D. Amidei et Al., The Silicon Vertex Detector of the Collider Detector at Fermilab, Nucl. Instr. Meth., A350 (1994), p. 73.

[5] K. Anikeev et AL., B Physics at the Tevatron Run II and Beyond. FERMILAB. Pub-01/197.

[6] G. Ascoli et AL., CDF Central Muon Detector, Nucl. Instr. Meth., A268 (1988), p. 33.

[7] P. Azzi et AL., SVX', the new CDF silicon vertex detector, Nucl. Instr. Meth., A360 (1995), p. 137.

[8] M. BAIley et AL., Measuring the b Quark Cross Sections Using the Exclusive Decays $B^{ \pm} \rightarrow J / \psi K^{ \pm}$and $B^{0} \rightarrow J / \psi K^{* 0}$. CDF Internal Note. 1994 .

[9] L. Balka ET AL., The CDF central electromagnetic calorimeter, Nucl. Instr. Meth., A267 (1988), p. 272.

[10] V. D. Barger and R. J. N. Phillips, Collider Physics, Addison-Wesley, 1996.

[11] F. Bedeschi et AL., Design and Construction of the CDF Central Tracking Chamber, Nucl. Instr. Meth., A268 (1988), p. 50.

[12] D. Benjamin et Al., Production of $J / \psi$ from $\chi_{c}$ decays at CDF. CDF Internal Note. 3124.

[13] D. Benjamin et Al., Production of $\Upsilon(1 P)$ from $\chi_{b}$ decay. CDF Internal Note. 4392.

[14] S. BertolucCi Et AL., The CDF central and endwall hadron calorimeters, Nucl. Instr. Meth., A267 (1988), p. 301.

[15] D. Boutigny et AL., The BaBar Physics Book. SLAC. r-504.

[16] G. Brandenburg et AL., An electromagnetic calorimeter for the small angle regions of the Collider Detector at Fermilab, Nucl. Instr. Meth., A267 (1988), p. 257. 
[17] J. T. Carroll et Al., The CDF Level 3 Trigger, Nucl. Instr. Meth., A300 (1991), p. 552 .

[18] CDF Collaboration, Measurement of the $B^{+}$Total Cross Section and $B^{+}$Differential Cross Section $d \sigma / d p_{t}$ in $p \bar{p}$ Collisions at $\sqrt{s}=1.8$ TeV, Phys. Rev. D.

[19] CDF Collaboration, Production of $J / \psi$ mesons from $\chi_{c}$ meson decays in $p \bar{p}$ collisions at $\sqrt{s}=1.8 T e V$, Phys. Rev. Lett., 79 (1997), p. 578.

[20] CDF Collaboration, Improved measurement of the $B^{-}$and $\overline{B^{0}}$ meson lifetimes using semileptonic decays, Phys. Rev. D, 58 (1998), p. 092002.

[21] CDF Collaboration, Production of $\Upsilon(1 S)$ mesons from $\chi_{b}$ decays in $\bar{p}$ collisions at $\sqrt{s}=1.8 T e V$, Phys. Rev. Lett., 84 (2000), p. 2094 .

[22] G. Cowan, Statistical Data Analysis, Oxford Science Publications, 1998.

[23] R. CRopp, A search for $h_{c}$ charmonium prooduction in the decay mode $J / \psi \pi^{0}$. CDF Internal Note. 4693.

[24] F. DeJongh et AL., Level 1 and Level 2 Low $p_{t}$ Central Muon Trigger Efficiencies for Run 1A. CDF Internal Note. 1999.

[25] D. Dong, Measurement of the b quark fragmentation function in $Z 0$ decays. SLAC, 1999. $\mathrm{r}-550$.

[26] H. T. Edwards, The Tevatron, Ann. Rev. Nucl. Sci., 35 (1985), pp. 605-660.

[27] T. Ferbel, Experimental Techniques in High Energy Physics, Addison-Wesley, 1987.

[28] R. Feynman and M. Gell-Mann, Phys. Rev., 109 (1958), p. 193.

[29] D. FreI, Multiple Scattering of Central Muons. CDF Internal Note. 1430.

[30] Y. FUKUI ET AL., CDF end plug electromagnetic calorimeter using conductive plastic proportional tubes, Nucl. Instr. Meth., A267 (1988), p. 280.

[31] S. L. Glashow, Nucl. Phys., 22 (1961), p. 579.

[32] D. GRIfFiths, Introduction to elementary particles, John Wiley \& Sons, 1987.

[33] D. E. Groom et Al., Review of Particle Properties, Eur. Phys. J., C15 (2000).

[34] S. R. HAhn ET AL., Calibration Systems for the CDF Central Electromagnetic Calorimeter, Nucl. Instr. Meth., A267 (1988), p. 351.

[35] R. M. HaRRIS ET AL., CES Response and $\chi^{2}$ for 1990 Testbeam Electrons. CDF Internal Note. 1432.

[36] R. M. HARRIS Et AL., Simulation of CES showers and $\chi^{2}$ in QFL. CDF Internal Note. 1222.

[37] Q. Ho-Kim and X. Pham, Elementary Particles and Their Interactions, SpringerVerlag, 1998. 
[38] K. D. Hoffman, The search for physics beyond the standard model in the b $\bar{b}$ spectrum observed in Tevatron proton-antiproton collisions, PhD thesis, Purdue University, 1998.

[39] T. A. KeAfFaber, Measurement of the $B^{+}$meson cross section in proton-antiproton collisions at $1.8 \mathrm{TeV}$ using the fully reconstructed decay $B^{+} \rightarrow J / \psi K^{+}, \mathrm{PhD}$ thesis, Purdue University, 2000.

[40] T. A. Keaffaber et AL., CTC Track Reconstruction Efficiency Studies. CDF Internal Note. 4768.

[41] M. Kobayashi and T. Maskawa, CP Violation In The Renormalizable Theory Of Weak Interaction, Prog. Theor. Phys., 49 (1973), p. 652.

[42] E. W. Kuns, A study of $W$ decay charge asymmetry using hadronic tau decays in proton-antiproton collisions at $\sqrt{s}=1.8 \mathrm{TeV}, \mathrm{PhD}$ thesis, Rutgers University, 1998.

[43] T. D. LeE, CERN Report. 61-30(1961).

[44] J. D. Lewis ET AL., The 1992 CDF Muon System Upgrade. CDF Internal Note. 2858 .

[45] M. Mangano, P. Nason, and G. Ridolfi, , Nucl. Phys., B373 (1992), p. 295.

[46] A. D. Martin, R. Roberts, and W. Stirling, New information on parton distributions, Phys. Rev. D, 47 (1993), p. 867.

[47] A. D. Martin, R. G. Roberts, W. J. Stirling, and R. S. Thorne, Parton Distributions: A New Global Analysis, Eur. Phys. J., C4 (1988), p. 463.

[48] S. P. PAPpas, DIMUTG: Dimuon Trigger Simulation for Run 1B. CDF Internal Note. 3537.

[49] M. Paulini and B. Wicklund, Summary of Proposals for B Physics Triggers in Run II. CDF Internal Note. 5483.

[50] C. Peterson, D. Schlatter, I. Schmitt, and P. M. Zerwas, Scaling Violations In Inclusive E+ E- Annihilation Spectra, Phys. Rev. D, 27 (1983), p. 105.

[51] K. PitTs. private communication, 2001.

[52] Y. SaKai et AL., Physics prospects of KEK asymmetric B-factory, Nucl. Instr. Meth., A333 (1993), p. 42.

[53] N. Solomey and A. B. Wicklund, Study of Electromagnetic Showers in the CDF Central Electromagnetic Calorimeter. CDF Internal Note. 0247.

[54] S. Stone Et Al., B Decays, World Scientific, 1994.

[55] W. J. TAYLOR, A Measurement of b-quark Fragmentation Fractions in $\bar{p} p$ Collisions at $\sqrt{s}=1.8 \mathrm{TeV}, \mathrm{PhD}$ thesis, University of Toronto, 1999 .

[56] A. B. WiCklund And L. Nodulman, Some software tools for using CES data. CDF Internal Note. 4036. 
[57] L. Wolfenstein, Parametrization Of The Kobayashi-Maskawa Matrix, Phys. Rev. Lett., 51 (1983), p. 1945.

[58] A. YAGIL, Fraction of $J / \psi$ coming from $\chi$, in $\bar{p} p$ Collisions at $\sqrt{s}=1.8 \mathrm{TeV}$. CDF Internal Note. 1526. 